\title{
Superoxide Radical Anion Adduct of 5,5-Dimethyl-1-pyrroline $N$-oxide (DMPO). 1. The Thermodynamics of Formation and Its Acidity
}

\author{
Frederick A. Villamena, ${ }^{*, \dagger}$ John Merle, ${ }^{\dagger}$ Christopher M. Hadad ${ }^{*}+\underset{\dagger}{ }$ and Jay L. \\ Zweier $^{*}+$ \\ ${ }^{\dagger}$ Center for Biomedical EPR Spectroscopy and Imaging, the Davis Heart and Lung \\ Research Institute, and the Division of Cardiovascular Medicine, Department of Internal \\ Medicine, College of Medicine, and ${ }^{\ddagger}$ Department of Chemistry, The Ohio State \\ University, Columbus, Ohio, 43210
}

\section{Supplementary Materials Table of Contents}

Complete References 46 and 47

S3

S4-S10

B3LYP/6-31+G(d,p)//B3LYP/6-31G(d) level (based on Figure 1)

Table S2. Thermodynamic data of the various conformations of $\mathrm{DMPO}-\mathrm{O}_{2}$ at the B3LYP/6-311+G(d,p) level (based on Figure 1)

Table S3. Thermodynamic data of the various conformations of the Conformation A of DMPO-O ${ }_{2} \mathrm{H}$ at the B3LYP/6-31+G(d,p)//B3LYP/6-31G(d) level

(based on Figure 2)

Table S4. Thermodynamic data of the various conformations of the Conformation $\mathrm{B}$ DMPO- ${ }_{2} \mathrm{H}$ at the B3LYP/6-31+G(d,p)//B3LYP/6-31G(d) level

Table S5. Total energies (in hartrees), $\Delta E_{0, \mathrm{rxn}}$ (in kcal/mol)a and Imaginary Vibrational Frequencies of DMPO- $\mathrm{O}_{2}$, and Conformations A and $\mathrm{B}$ of of DMPO-O $\mathrm{H}_{2}$ at the PCM/B3LYP/6-31+G(d,p)//B3LYP/6-31G(d) level. (Based on Figure 3)

Figure S1. Rotational barriers in the DMPO-O $2^{-}(\bullet)$, DMPO-O2H conformation A $(\triangle)$ and DMPO-O2H conformation B $(\boldsymbol{\Delta})$. The dihedral angles are at 22.50 increments along the N-C-O-O angle as a function of the bottom-of-the-well energies $(\Delta E 0, \mathrm{rxn}) \mathrm{in} \mathrm{kcal} / \mathrm{mol}$ at the PCM B3LYP/6 31+G(d,p) //B3LYP/6-31G(d) level of theory using a dielectric constant for water.

Table S6. Thermodynamic data of various acids at the PCM/B3LYP/6-311+G(d)// B3LYP/6-311+G(d) level (based on Table 2)

Figure S2. Plot of the experimental $\mathrm{pKa}$ versus the calculated $\mathrm{pKa}$. See Table 2 for the thermodynamic parameters.

\footnotetext{
* Correspondence to: villamena-1@medctr.osu-edu, Fax: (614)-292-8454; hadad.1@osu.edu, Fax: (614)292-1685; zweier-1@medctr.osu.edu, Fax: (614)-247-7799.
} 
Table S7. Thermodynamic data for the mechanism DMPO-OOH formation at the B3LYP/6-311+G(d) and PCM/B3LYP/6-311+G(d)//B3LYP/6-311+G(d) level (based on Table 3)

Table S8. Transition state data for the DMPO-OOH formation at the B3LYP $/ 631+\mathrm{G}$ $(\mathrm{d}, \mathrm{p}) / / \mathrm{B} 3 \mathrm{LYP} / 6-31 \mathrm{G}(\mathrm{d})$ level in gas and aqueous phases (based on Table 4)

Table S9.Thermodynamic Parameters for the O-H Bond Dissociation of TEMPO-OH at the B3LYP/6-31+G(d,p)//B3LYP/6-31G(d), CBS-Q and CBS-QB3 levels in gas phase and CBS-QB3 Energies of the 3 Major DMPO-OOH Conformations 


\section{Complete References}

(46) Frisch, M. J.; Trucks, G. W.; Schlegel, H. B.; Scuseria, G. E.; Robb, M. A.; Cheeseman, J. R.; Zakrzewski, V. G.; Montgomery, J., J. A. ; Stratmann, R. E.; Burant, J. C.; Dapprich, S.; Millam, J. M.; Daniels, A. D.; Kudin, K. N.; Strain, M. C.; Farkas, O.; Tomasi, J.; Barone, V.; Cossi, M.; Cammi, R.; Mennucci, B.; Pomelli, C.; Adamo, C.; Clifford, S.; Ochterski, J.; Petersson, G. A.; Ayala, P. Y.; Cui, Q.; Morokuma, K.; Rega, N.; Salvador, P.; Dannenberg, J. J.; Malick, D. K.; Rabuck, A. D.; Raghavachari, K.; Foresman, J. B.; Cioslowski, J.; Ortiz, J. V.; Baboul, A. G.; Stefanov, B. B.; Liu, G.; Liashenko, A.; Piskorz, P.; Komaromi, I.; Gomperts, R.; Martin, R. L.; Fox, D. J.; Keith, T.; Al-Laham, M. A.; Peng, C. Y.; Nanayakkara, A.; Challacombe, M.; Gill, P. M. W.; Johnson, B.; Chen, W.; Wong, M. W.; Andres, J. L.; Gonzalez, C.; Head-Gordon, M.; Replogle, E. S.; Pople, J. A. Gaussian 98; Revision A.11.3; Gaussian, Inc.: Pittsburgh PA, 2002.

(47) Frisch, M. J.; Trucks, G. W.; Schlegel, H. B.; Scuseria, G. E.; Robb, M. A.; Cheeseman, J. R.; Montgomery, J. A., Jr.; T. Vreven; Kudin, K. N.; Burant, J. C.; Millam, J. M.; Iyengar, S. S.; Tomasi, J.; Barone, V.; Mennucci, B.; Cossi, M.; Scalmani, G.; Rega, N.; Petersson, G. A.; Nakatsuji, H.; Hada, M.; Ehara, M.; Toyota, K.; Fukuda, R.; Hasegawa, J.; Ishida, M.; Nakajima, T.; Honda, Y.; Kitao, O.; Nakai, H.; Klene, M.; Li, X.; Knox, J. E.; Hratchian, H. P.; Cross, J. B.; Adamo, C.; Jaramillo, J.; Gomperts, R.; Stratmann, R. E.; Yazyev, O.; Austin, A. J.; Cammi, R.; Pomelli, C.; Ochterski, J. W.; Ayala, P. Y.; Morokuma, K.; Voth, G. A.; Salvador, P.; Dannenberg, J. J.; Zakrzewski, V. G.; Dapprich, S.; Daniels, A. D.; Strain, M. C.; Farkas, O.; Malick, D. K.; Rabuck, A. D.; Raghavachari, K.; Foresman, J. B.; Ortiz, J. V.; Cui, Q.; Baboul, A. G.; Clifford, S.; Cioslowski, J.; Stefanov, B. B.; Liu, G.; Liashenko, A.; Piskorz, P.; Komaromi, I.; Martin, R. L.; Fox, D. J.; Keith, T.; Al-Laham, M. A.; Peng, C. Y.; Nanayakkara, A.; Challacombe, M.; Gill, P. M. W.; Johnson, B.; Chen, W.; Wong, M. W.;

Gonzalez, C.; Pople, J. A. Gaussian 03; Revision B.04; Gaussian, Inc.: Pittsburgh PA, 2003. 


\section{Table S1}

Thermodynamic data of the various conformations of DMPO- $\mathrm{O}_{2}$ at the B3LYP/6-31+G(d,p)//B3LYP/6-31G(d) level (based on Figure 1) 
b3lyp/6-31+g**//b3lyp/6-31g*

ZPE scaling factor $=$
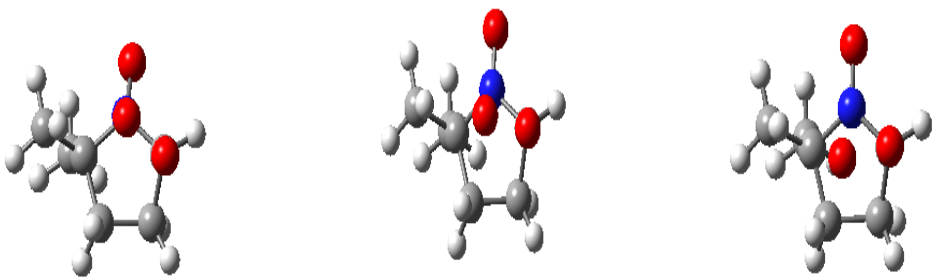

Compound
file
E 6-31+g** tight
E 6-31g*
ZPE (raw)
Therm Corr to H (raw)
Therm Corr to G (raw)
H (raw)
G (raw)
ZPE (scaled)
Electronic State
s2 6-31g*
Symmetry
N Imag
Rotational Con. (GHz)
Rotational Con. (GHz)
Rotational Con. (GHz)
H298
6-31+g** tight
6-31g*
G298
6-31+g** tight
6-31g*

\begin{tabular}{|c|c|c|}
\hline \multirow{2}{*}{$\begin{array}{l}\text { DMPO-02 } 360 \text { deg } \\
\text { o2dpKurp_0b.log }\end{array}$} & DMPO-O2 $337.5 \mathrm{deg}$ & DMPO-O2 315 deg \\
\hline & o2dpKurp_22_5F.log & o2dpKurp_45F.log \\
\hline-515.565501 & -515.570241 & -515.5775193 \\
\hline-515.503071 & -515.5085433 & -515.5166163 \\
\hline 0.171005 & 0.171065 & 0.171528 \\
\hline 0.181497 & 0.181572 & 0.182743 \\
\hline 0.136309 & 0.136412 & 0.135916 \\
\hline-515.321574 & -515.326972 & -515.333874 \\
\hline-515.366762 & -515.372131 & -515.3807 \\
\hline 0.167688 & 0.167746 & 0.168200 \\
\hline 0.7541 & 0.754 & 0.7538 \\
\hline C1 & $\mathrm{C} 1$ & $\mathrm{C} 1$ \\
\hline 1 & 1 & 0 \\
\hline 1.84821 & 1.88651 & 1.90912 \\
\hline 1.23321 & 1.21584 & 1.21042 \\
\hline 1.17669 & 1.17672 & 1.15848 \\
\hline-515.387322 & -515.391988 & -515.398104 \\
\hline-515.324892 & -515.330290 & -515.337201 \\
\hline-515.432510 & -515.437148 & -515.444931 \\
\hline-515.370080 & -515.375450 & -515.384028 \\
\hline
\end{tabular}




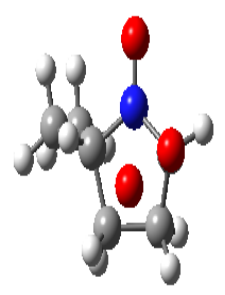

Compound
file
E 6-31+g** tight
E 6-31g*
ZPE (raw)
Therm Corr to H (raw)
Therm Corr to G (raw)
H (raw)
G (raw)
ZPE (scaled)
Electronic State
s2 6-31g*
Symmetry
N Imag
Rotational Con. (GHz)
Rotational Con. (GHz)
Rotational Con. (GHz)
H298
6-31+g** tight
6-31g*
G298
6-31+g** tight
6-31g*

\begin{tabular}{|c|c|c|}
\hline DMPO-O2 $292.5 \mathrm{deg}$ & DMPO-O2 $270 \mathrm{deg}$ & DMPO-O2 $247.5 \mathrm{deg}$ \\
\hline o2dpKurp_67_5F.log & o2dpKurp_90F.log & o2dpKurp_112_5F.log \\
\hline-515.5814617 & -515.57997 & -515.5762396 \\
\hline-515.5215133 & -515.5209548 & -515.5164896 \\
\hline 0.171891 & 0.171834 & 0.171328 \\
\hline 0.182924 & 0.182818 & 0.181707 \\
\hline 0.136609 & 0.136549 & 0.136568 \\
\hline-515.33859 & -515.338137 & -515.334783 \\
\hline-515.384905 & -515.384406 & -515.379921 \\
\hline 0.168556 & 0.168500 & 0.168004 \\
\hline 0.7537 & 0.754 & 0.755 \\
\hline C1 & C1 & $\mathrm{C} 1$ \\
\hline 0 & 0 & 1 \\
\hline 1.919610 & 1.926150 & 1.951170 \\
\hline 1.214360 & 1.217990 & 1.209440 \\
\hline 1.130820 & 1.112710 & 1.100350 \\
\hline-515.401872 & -515.400486 & -515.397856 \\
\hline-515.341924 & -515.341470 & -515.338106 \\
\hline-515.448187 & -515.446755 & -515.442995 \\
\hline-515.388239 & -515.387739 & -515.383245 \\
\hline
\end{tabular}
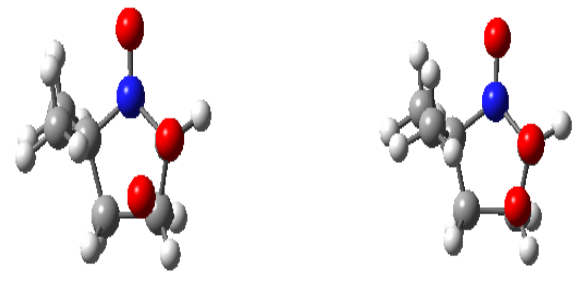


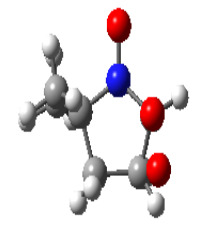

Compound
file
E 6-31+g** tight
E 6-31g*
ZPE (raw)
Therm Corr to H (raw)
Therm Corr to G (raw)
H (raw)
G (raw)
ZPE (scaled)
Electronic State
s2 6-31g*
Symmetry
N Imag
Rotational Con. (GHz)
Rotational Con. (GHz)
Rotational Con. (GHz)
H298
6-31+g** tight
6-31g*
G298
6-31+g** tight
6-31g*

\begin{tabular}{|c|c|c|}
\hline \multirow{2}{*}{$\begin{array}{l}\text { DMPO-O2 } 225 \text { deg } \\
\text { o2dpKurp 135F.log }\end{array}$} & DMPO-O2 $202.5 \mathrm{deg}$ & DMPO-O2 $180 \mathrm{deg}$ \\
\hline & o2dpKurp_157_5F.log & o2dpKurp_180F.log \\
\hline-515.5762343 & -515.5786199 & -515.5790119 \\
\hline-515.5139652 & -515.5151319 & -515.5145645 \\
\hline 0.170873 & 0.171082 & 0.171175 \\
\hline 0.181378 & 0.182396 & 0.18245 \\
\hline 0.1361 & 0.134683 & 0.135185 \\
\hline-515.332587 & -515.332736 & -515.332114 \\
\hline-515.377865 & -515.380449 & -515.379379 \\
\hline 0.167558 & 0.167763 & 0.167854 \\
\hline 0.7566 & 0.7574 & 0.7571 \\
\hline $\mathrm{C} 1$ & $\mathrm{C} 1$ & C1 \\
\hline 1 & 0 & 0 \\
\hline 2.04352 & 2.12809 & 2.19308 \\
\hline 1.14199 & 1.09272 & 1.05496 \\
\hline 1.03403 & 0.98224 & 0.94119 \\
\hline-515.398171 & -515.399543 & -515.399883 \\
\hline-515.335902 & -515.336055 & -515.335435 \\
\hline-515.443449 & -515.447256 & -515.447148 \\
\hline-515.381180 & -515.383768 & -515.382700 \\
\hline
\end{tabular}




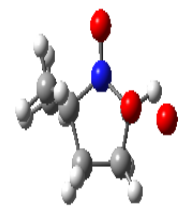

Compound
file
E 6-31+g** tight
E 6-31g*
ZPE (raw)
Therm Corr to H (raw)
Therm Corr to G (raw)
H (raw)
G (raw)
ZPE (scaled)
Electronic State
s2 6-31g*
Symmetry
N Imag
Rotational Con. (GHz)
Rotational Con. (GHz)
Rotational Con. (GHz)
H298
6-31+g** tight
6-31g*
G298
6-31+g** tight
6-31g*
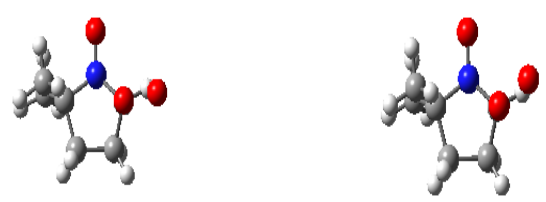

\begin{tabular}{|c|c|c|c|}
\hline \multicolumn{2}{|c|}{$\begin{array}{l}\text { DMPO-O2 } 157.5 \text { deg } \\
\text { o2dpKurp_202_5F.log }\end{array}$} & DMPO-O2 $135 \mathrm{deg}$ & $\begin{array}{l}\text { DMPO-O2 } 112.5 \mathrm{deg} \\
02 \mathrm{dnKurn} 2475 \mathrm{~F}] \mathrm{g}\end{array}$ \\
\hline & -515.5754513 & -515.5718422 & -515.5725587 \\
\hline & -515.5081503 & -515.5064573 & -515.5085046 \\
\hline & 0.170708 & 0.170525 & 0.170692 \\
\hline & 0.181445 & 0.181152 & 0.181273 \\
\hline & 0.135063 & 0.135414 & 0.135647 \\
\hline & -515.326705 & -515.325305 & -515.327232 \\
\hline & -515.373087 & -515.371044 & -515.372858 \\
\hline & 0.167396 & 0.167217 & 0.167381 \\
\hline & 0.7549 & 0.7582 & 0.7574 \\
\hline $\mathrm{C} 1$ & & $\mathrm{C} 1$ & $\mathrm{C} 1$ \\
\hline & 1 & 1 & \\
\hline & 2.198990 & 2.145830 & 2.089840 \\
\hline & 1.030180 & 1.045950 & 1.082910 \\
\hline & 0.921140 & 0.929910 & 0.948600 \\
\hline & -515.397318 & -515.393998 & -515.394597 \\
\hline & -515.330017 & -515.328614 & -515.330543 \\
\hline & -515.443700 & -515.439736 & -515.440223 \\
\hline & -515.376399 & -515.374352 & -515.37616 \\
\hline
\end{tabular}




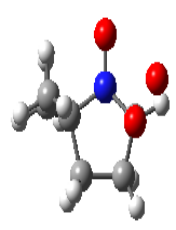

\begin{tabular}{|c|c|c|}
\hline $\begin{array}{l}\text { DMPO-02 } 90 \text { deg } \\
\text { o2dpKurp_270.log }\end{array}$ & $\begin{array}{l}\text { DMPO-02 } 67.5 \mathrm{deg} \\
\text { o2dpKurp_292_5F.log }\end{array}$ & $\begin{array}{l}\text { DMPO-02 } 45 \text { deg } \\
\text { o2dpKurp_315F.log }\end{array}$ \\
\hline-515.5740351 & -515.57332 & -515.5691967 \\
\hline-515.5109172 & -515.5116875 & -515.509310 \\
\hline 0.171025 & 0.171131 & 0.171265 \\
\hline 0.18237 & 0.182435 & 0.182325 \\
\hline 0.134635 & 0.134903 & 0.135979 \\
\hline-515.328547 & -515.329252 & -515.326985 \\
\hline-515.376283 & -515.376784 & -515.373331 \\
\hline 0.167707 & 0.167811 & 0.167942 \\
\hline 0.756300 & 0.755600 & 0.754800 \\
\hline C1 & C1 & $\mathrm{C} 1$ \\
\hline 0 & 0 & \\
\hline 2.02104 & 1.94347 & 1.863540 \\
\hline 1.15413 & 1.25734 & 1.322700 \\
\hline 0.98881 & 1.05305 & 1.112990 \\
\hline-515.394983 & -515.394205 & -515.390194 \\
\hline-515.331865 & -515.332572 & -515.330307 \\
\hline-515.442718 & -515.441737 & -515.436540 \\
\hline-515.379600 & -515.380104 & -515.376653 \\
\hline
\end{tabular}
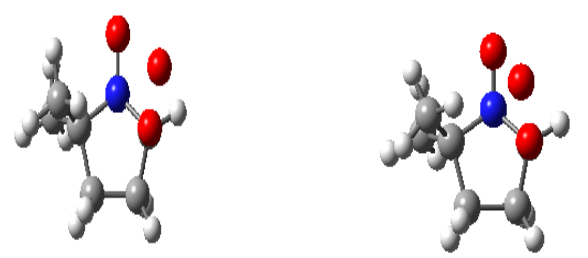

Compound
file
E 6-31+g** tight
E 6-31g*
ZPE (raw)
Therm Corr to H (raw)
Therm Corr to G (raw)
H (raw)
G (raw)
ZPE (scaled)
Electronic State
s2 6-31g*
Symmetry
N Imag
Rotational Con. (GHz)
Rotational Con. (GHz)
Rotational Con. (GHz)
H298
6-31+g** tight
6-31g*
G298
6-31+g** tight
6-31g*




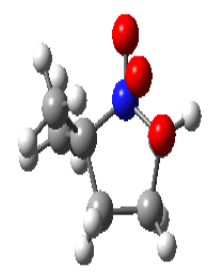

Compound
file
E 6-31+g** tight
E 6-31g*
ZPE (raw)
Therm Corr to H (raw)
Therm Corr to G (raw)
H (raw)
G (raw)
ZPE (scaled)
Electronic State
s2 6-31g*
Symmetry
N Imag
Rotational Con. (GHz)
Rotational Con. (GHz)
Rotational Con. (GHz)
H298
6-31+g** tight
6-31g*
G298
6-31+g** tight
6-31g*

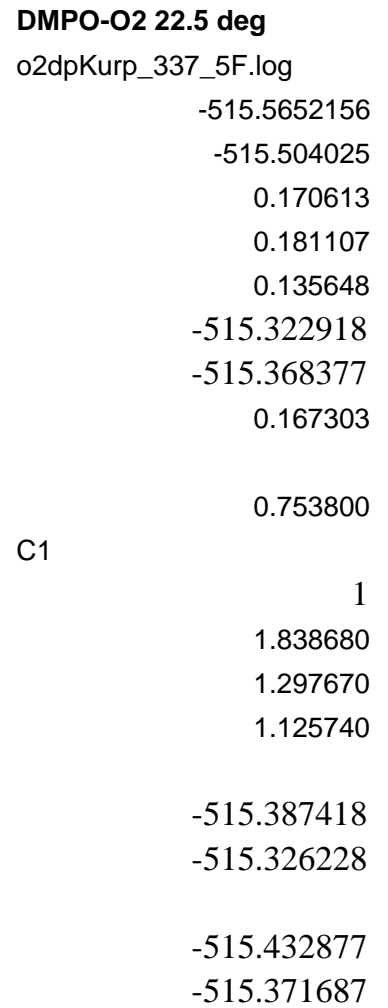




\section{Table S2}

Thermodynamic data of the various conformations of DMPO- $\mathrm{O}_{2}$ at the B3LYP/6-311+G(d,p) level (based on Figure 1) 
b3lyp/6-311+g**

ZPE scaling factor $=$

\section{Compound}

file

E 6-311+g**

ZPE (raw)

Therm Corr to $\mathbf{H}$ (raw)

Therm Corr to $\mathbf{G}$ (raw)

H (raw)

G (raw)

ZPE (scaled)

Electronic State

s2 6-31g*

Symmetry

N Imag

Rotational Con. (GHz)

Rotational Con. (GHz)

Rotational Con. (GHz)

H298

$6-311+g^{* \star}$

$6-311+g^{* *}$

G298

6-311+g**

$6-311+g^{* *}$
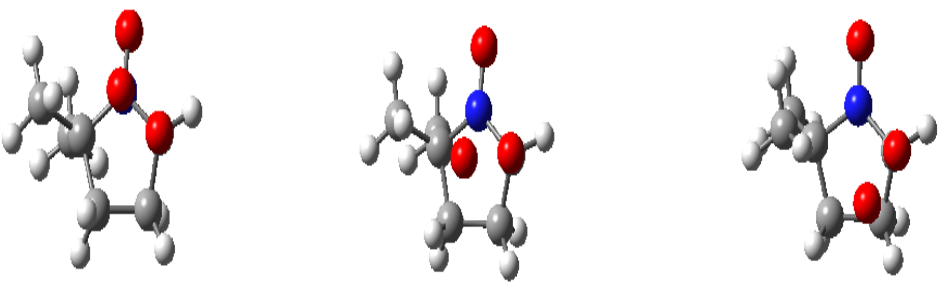

DMPO-O2 360 degrees

o2dpKurp_Otzb.log
DMPO-02 315 degrees

o2dpKurp_45tzb.log

$-515.6952327$

0.169672

0.181022

0.133864

$-515.514211$

$-515.561368$

0.7547

C1

$C 1$
$1.000000-$
1.850250
1.228390
1.150500
0.010699
-515.672498
-0.034989
-515.718186

0.7547

$\begin{array}{rr}\text { C1 } & 0 \\ 1.911410 & 1.92626 \\ 1.199910 & 1.19843 \\ 1.143910 & 1.09581 \\ & \\ 0.011350 & 0.011263 \\ -515.683883 & -515.686409 \\ -0.035808 & -0.035937 \\ -515.731041 & -515.733609\end{array}$

DMPO-O2 270 degrees

o2dpKurp_90tz.log

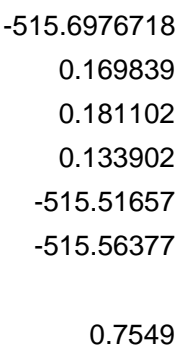

$-515.6976718$

0.169839

0.181102

0.133902

$-515.51657$

$-515.56377$

0.7549 .

92626

1.19843

.09581

63 


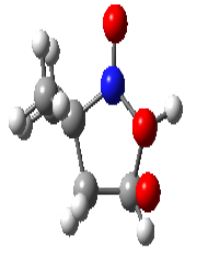

Compound

file

E 6-311+g**
ZPE (raw)
Therm Corr
Therm Corr
H (raw)
G (raw)
ZPE (scaled)
Electronic Sta
s2 6-31g*
Symmetry
N Imag
Rotational Co
Rotational Co
Rotational Co
H298
$6-311+g^{\star *}$
$6-311+g^{\star *}$
G298
$6-311+g^{\star *}$
$6-311+g^{\star *}$

DMPO-02 225 degrees

o2dpKurp_135tz.log

$-515.6938454$

0.169058

0.179734

0.133837

$-515.514111$

$-515.560008$

0.757

C1

-

$C 1$
2.047400
1.122480
1.015170
0.010676
-515.683169
-0.035221
-515.729066

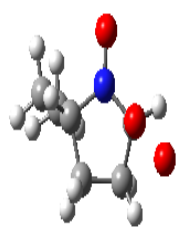

DMPO-O2 180 degrees

o2dpKurp_180tz.log

$-515.696844$

0.169458

0.180922

0.133029

$-515.515922$

$-515.563815$

0.7561

C1

0.000000

2.224790

1.020660

0.910250

0.011464

$-515.685380$

$-0.036429$

$-515.733273$

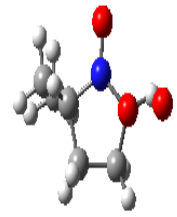

DMPO-O2 135 degrees

o2dpKurp_225tz.log

-515.6899808
0.169012
0.179806
0.133372
-515.510175
-515.556609
0.7569

(1)

1
2.18586
1.01189
0.90022

0.010794
-515.679187
-0.035640
-515.725621




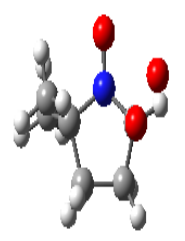

Compound
file
E 6-311+g**
ZPE (raw)
Therm Corr to H (raw)
Therm Corr to G (raw)
H (raw)
G (raw)
ZPE (scaled)
Electronic State
s2 6-31g*
Symmetry
N Imag
Rotational Con. (GHz)
Rotational Con. (GHz)
Rotational Con. (GHz)
H298
6-311+g**
6-311+g**
G298
6-311+g**
$6-311+g^{\star *}$

C1
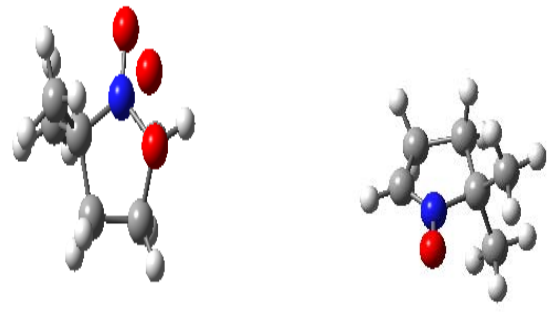

DMPO-02 90 degrees

o2dpKurp_270tz.log

DMPO-O2 45 degrees

DMPO

o2dpKurp_315tzb.log dmpotz.log

$-515.6920645$

0.169191

0.18068

0.132703

$-515.511384$

$-515.559362$

0.7574

0.000000
2.022000
1.125080
0.975730
0.011489
15.680576

$-0.036488$

$-515.728553$

$\begin{array}{rr}-515.6879905 & -365.2835638 \\ 0.169105 & 0.165016 \\ 0.180719 & 0.173973 \\ 0.131945 & 0.133191 \\ -515.507272 & -365.109591 \\ -515.556045 & -365.150373 \\ 0.7549 & 0\end{array}$

$0.000000-$

1.845980 -

$1.250150-$

1.100750 -

0.011614

$-515.676376$

$-0.037160$

$-515.725150$ 


\section{0}

\begin{tabular}{|c|c|c|}
\hline \multicolumn{3}{|l|}{ Compound } \\
\hline file & o2tz.log & \\
\hline$E$ 6-311 $+g^{* *}$ & & -150.3917078 \\
\hline ZPE (raw) & & 0.002655 \\
\hline Therm Corr to H (raw) & & 0.005979 \\
\hline Therm Corr to G (raw) & & -0.017138 \\
\hline H (raw) & & -150.385729 \\
\hline G (raw) & & -150.408846 \\
\hline \multicolumn{3}{|l|}{ ZPE (scaled) } \\
\hline Electronic State & & 0.7565 \\
\hline \multicolumn{3}{|l|}{ s2 6-31g* } \\
\hline Symmetry & - & \\
\hline N Imag & & 0.000000 \\
\hline Rotational Con. (GHz) & - & \\
\hline Rotational Con. (GHz) & - & \\
\hline Rotational Con. (GHz) & - & \\
\hline \multicolumn{3}{|l|}{ H298 } \\
\hline \multicolumn{3}{|l|}{$6-311+g^{\star *}$} \\
\hline \multicolumn{3}{|l|}{$6-311+g^{\star *}$} \\
\hline \multicolumn{3}{|l|}{ G298 } \\
\hline \multicolumn{3}{|l|}{$6-311+g^{\star *}$} \\
\hline $6-311+g^{* *}$ & & \\
\hline
\end{tabular}



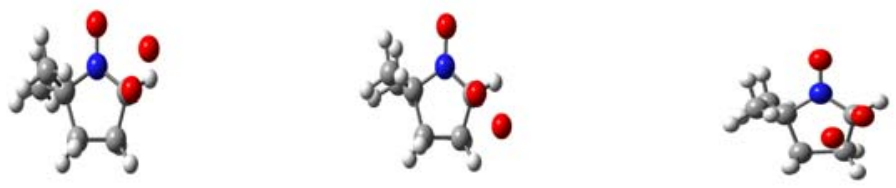

\begin{tabular}{|c|c|c|c|}
\hline Compound & DMPO-O2 82.20 & DMPO-O2 187.30 & DMPO-O2 289.30 \\
\hline file & 02dptz_292_5.log & 02dptz_180freq.log & 02dptz_67_5.log \\
\hline E 6-311+g** tight (PCM) & -515.793405 & -515.79343 & -515.791361 \\
\hline$E$ 6-311 $+g^{\star *}$ & -515.6922488 & -515.6970753 & -515.6993685 \\
\hline ZPE (raw) & 0.169227 & 0.169407 & 0.169941 \\
\hline Therm Corr to $\mathrm{H}$ (raw) & 0.180698 & 0.180853 & 0.181186 \\
\hline Therm Corr to G (raw) & 0.132828 & 0.132976 & 0.134219 \\
\hline H (raw) & -515.511551 & -515.516222 & -515.518183 \\
\hline G (raw) & -515.55942 & -515.564099 & -515.56515 \\
\hline ZPE (scaled) & 0.165944 & 0.166121 & 0.166644 \\
\hline \multicolumn{4}{|l|}{ Electronic State } \\
\hline s2 6-31g* & 0.7571 & 0.7566 & 0.7547 \\
\hline Symmetry & - & $\mathrm{C} 1$ & \\
\hline N Imag & 0 & 0 & 0 \\
\hline Rotational Con. (GHz) & - & - & - \\
\hline Rotational Con. (GHz) & - & - & - \\
\hline Rotational Con. (GHz) & - & - & - \\
\hline \multicolumn{4}{|l|}{ H298 } \\
\hline H 6-311+g ${ }^{* *}$ tight PCM & -515.615990 & -515.615863 & -515.613472 \\
\hline H 6-311+g** & -515.514834 & -515.519509 & -515.521479 \\
\hline \multicolumn{4}{|l|}{ G298 } \\
\hline G 6-311+g** tight (PCM) & -515.663860 & -515.663740 & -515.660439 \\
\hline G 6-311+g** & -515.562704 & -515.567386 & -515.568446 \\
\hline DG Solvation & -63.4764859 & -60.46346013 & -57.72615199 \\
\hline
\end{tabular}




\section{Table S3}

Thermodynamic data of the various conformations of the Conformation A of DMPO- $\mathrm{O}_{2} \mathrm{H}$ at the B3LYP/6-31+G(d,p)//B3LYP/6-31G(d) level

(based on Figure 2) 
b3lyp/6-31+g**//b3lyp/6-31 g*

ZPE scaling factor $=$
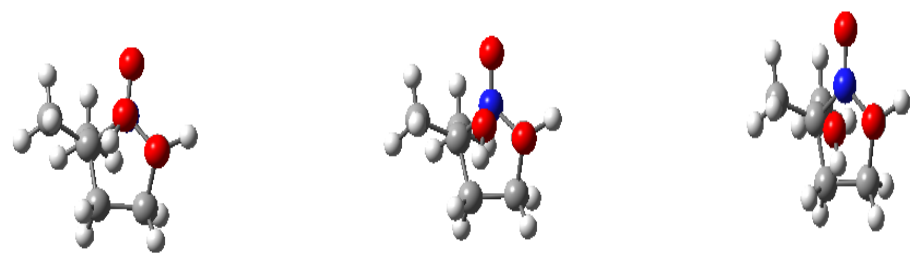

\begin{tabular}{|c|c|c|}
\hline $\begin{array}{l}\text { DMPO-O2H } 360 \text { deg } \\
\text { oxdp_0.log }\end{array}$ & $\begin{array}{l}\text { DMPO-O2H } 337.5 \text { deg } \\
\text { oxdp_22_5.log }\end{array}$ & $\begin{array}{l}\text { DMPO-O2H } 315 \text { deg } \\
\text { oxdp_45.log }\end{array}$ \\
\hline-516.151138 & -516.154026 & -516.159161 \\
\hline-516.1082566 & -516.1110361 & -516.1161514 \\
\hline 0.184548 & 0.18469 & 0.185046 \\
\hline 0.19576 & 0.195833 & 0.196904 \\
\hline 0.148953 & 0.149217 & 0.148569 \\
\hline-515.912497 & -515.915203 & -515.919248 \\
\hline-515.959303 & -515.961819 & -515.967582 \\
\hline 0.180968 & 0.181107 & 0.181456 \\
\hline 0.7534 & 0.7534 & 0.7534 \\
\hline C1 & $\mathrm{C} 1$ & C1 \\
\hline 1 & 1 & 0 \\
\hline 1.87906 & 1.89682 & 1.92371 \\
\hline 1.19617 & 1.18165 & 1.15579 \\
\hline 1.06287 & 1.07819 & 1.07652 \\
\hline-515.958959 & -515.961776 & -515.965847 \\
\hline-515.916077 & -515.918786 & -515.922837 \\
\hline-516.005766 & -516.008392 & -516.014182 \\
\hline-515.962884 & -515.965402 & -515.971172 \\
\hline
\end{tabular}




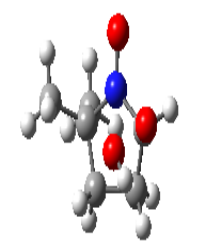

Compound
file
E 6-31+g** tight
E 6-31 g*
ZPE (raw)
Therm Corr to H (raw)
Therm Corr to G (raw)
H (raw)
G (raw)
ZPE (scaled)
Electronic State
s2 6-31 g*
Symmetry
N Imag
Rotational Con. (GHz)
Rotational Con. (GHz)
Rotational Con. (GHz)
H298
6-31+g** tight
6-31 g*
G298
6-31+g** tight
6-31 g*

\begin{tabular}{|c|c|c|}
\hline $\begin{array}{l}\text { DMPO-O2H } 292.5 \text { deg } \\
\text { oxdp_67_5.log }\end{array}$ & $\begin{array}{l}\text { DMPO-O2H } 270 \text { deg } \\
\text { oxdp_90.log }\end{array}$ & $\begin{array}{l}\text { DMPO-O2H } 247.5 \text { deg } \\
\text { oxdp_112.5.log }\end{array}$ \\
\hline-516.1614961 & -516.1589909 & -516.1552713 \\
\hline-516.1183491 & -516.1157038 & -516.1119055 \\
\hline 0.185144 & 0.184989 & 0.184782 \\
\hline 0.197018 & 0.196942 & 0.195911 \\
\hline 0.148403 & 0.147895 & 0.148814 \\
\hline-515.921331 & -515.918762 & -515.915994 \\
\hline-515.969946 & -515.967809 & -515.963092 \\
\hline 0.181552 & 0.181400 & 0.181197 \\
\hline 0.7533 & 0.7534 & 0.7534 \\
\hline C1 & $\mathrm{C} 1$ & $\mathrm{C} 1$ \\
\hline 0 & 0 & 1 \\
\hline 1.957350 & 1.994740 & 2.023710 \\
\hline 1.124880 & 1.092380 & 1.076420 \\
\hline 1.039490 & 0.990540 & 0.967500 \\
\hline-515.968070 & -515.965638 & -515.962945 \\
\hline-515.924923 & -515.922351 & -515.919579 \\
\hline-516.016685 & -516.014685 & -516.010042 \\
\hline-515.973538 & -515.971398 & -515.966676 \\
\hline
\end{tabular}

DMPO-O2H 247.5 deg

.log

$-516.1552713$

1119055

184782

0.195911

0.148814

$-515.915994$

963092

.7534

1

.023710
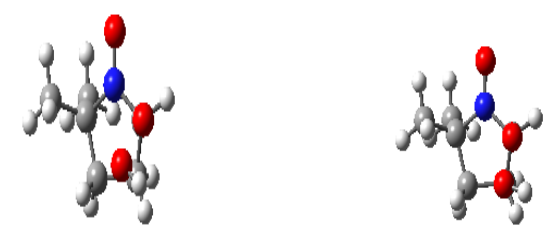

.




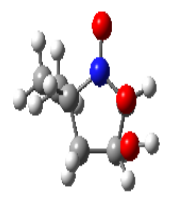

Compound
file
E 6-31+g** tight
E 6-31 g*
ZPE (raw)
Therm Corr to H (raw)
Therm Corr to G (raw)
H (raw)
G (raw)
ZPE (scaled)
Electronic State
s2 6-31 g*
Symmetry
N Imag
Rotational Con. (GHz)
Rotational Con. (GHz)
Rotational Con. (GHz)
H298
6-31+g** tight
6-31 g*
G298
6-31+g** tight
6-31 g*

\begin{tabular}{|c|c|c|c|}
\hline \multirow[t]{2}{*}{$\begin{array}{l}\text { DMPO-O2H } \\
\text { oxdp_135.log }\end{array}$} & 225 deg & $\begin{array}{l}\text { DMPO-O2H } 202.5 \text { deg } \\
\text { oxdp_157_5.log }\end{array}$ & $\begin{array}{l}\text { DMPO-O2H } 180 \text { deg } \\
\text { oxdp_180.log }\end{array}$ \\
\hline & -516.1554955 & -516.1593722 & -516.1624819 \\
\hline & -516.1120772 & -516.1157505 & -516.1186531 \\
\hline & 0.184811 & 0.184957 & 0.18508 \\
\hline & 0.195911 & 0.196878 & 0.196926 \\
\hline & 0.149033 & 0.147795 & 0.148433 \\
\hline & -515.916166 & -515.918872 & -515.921727 \\
\hline & -515.963044 & -515.967955 & -515.97022 \\
\hline & 0.181226 & 0.181369 & 0.181489 \\
\hline & 0.7535 & 0.7534 & 0.7533 \\
\hline $\mathrm{C} 1$ & & $\mathrm{C} 1$ & $\mathrm{C} 1$ \\
\hline & 1 & 0 & 0 \\
\hline & 2.06197 & 2.13918 & 2.21093 \\
\hline & 1.06411 & 1.02844 & 0.99454 \\
\hline & 0.9584 & 0.92295 & 0.88607 \\
\hline & -515.963170 & -515.966082 & -515.969146 \\
\hline & -515.919752 & -515.922461 & -515.925318 \\
\hline & -516.010048 & -516.015165 & -516.017639 \\
\hline & -515.966630 & -515.971544 & -515.973811 \\
\hline
\end{tabular}




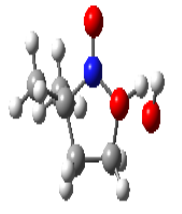

Compound
file
E 6-31+g** tight
E 6-31 g* $^{*}$
ZPE (raw)
Therm Corr to H (raw)
Therm Corr to G (raw)
H (raw)
G (raw)
ZPE (scaled)
Electronic State
s2 6-31 g*
Symmetry
N Imag
Rotational Con. (GHz)
Rotational Con. (GHz)
Rotational Con. (GHz)
H298
6-31+g** tight
6-31 g*
G298
6-31+g** tight
6-31 g*

oxdp_202_5.log

C1

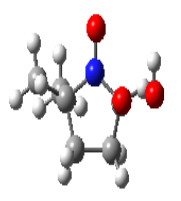

DMPO-O2H $157.5 \mathrm{deg}$ $-516.1621066$ $-516.118061$

0.18492

0.196882

0.147209

$-515.921179$

$-515.970852$

0.181333

0.7533

0
2.238630
0.974270
0.862440
-515.968812
-515.924766
-516.018485
-515.974439

DMPO-O2H 135 deg

oxdp_225.log

$-516.1612855$

$-516.1171804$

0.184799

0.195886

0.148933

$-515.921295$

$-515.968247$

0.181214

DMPO-O2H 112.5 deg

oxdp_247_5.log

$-516.1624604$

$-516.1188818$

0.185002

0.196012

0.149188

$-515.92287$

$-515.969694$

0.181413

0.7534

C1

0

0.7535

C1

1

2.227200

0.975190

0.855590

$-515.968985$

$-515.924879$

$-515.970037$

$-515.926459$

$-516.015938$

$-515.971832$
$-516.016861$

$-515.973283$ 


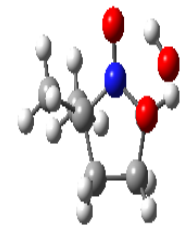

\section{DMPO-O2H 90 deg}

oxdp_270.log

file

E 6-31+g** tight

E 6-31 $\mathrm{g}^{*}$

ZPE (raw)

Therm Corr to $\mathrm{H}$ (raw)

Therm Corr to $\mathrm{G}$ (raw)

$H$ (raw)

G (raw)

ZPE (scaled)

Electronic State

s2 6-31 g*

Symmetry

$N$ Imag

Rotational Con. (GHz)

Rotational Con. (GHz)

Rotational Con. (GHz)

H298

6-31+g** tight

6-31 $\mathrm{g}^{*}$

G298

6-31+g** tight

6-31 $\mathrm{g}^{*}$

C1

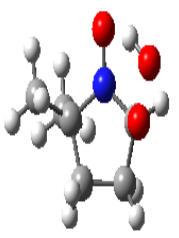

DMPO-O2H $67.5 \mathrm{deg}$

oxdp_292_5.log

$-516.1656566$

$-516.1235143$

0.185662

0.197266

0.149006

$-515.926248$

$-515.974508$

0.182060

0.7537

C1

0

2.13861

1.07177

0.90053

$-515.971992$

$-515.929850$

$-516.020252$

$-515.978110$

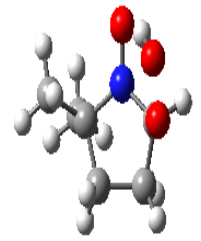

DMPO-O2H 45 deg

oxdp_315.log

$-516.1592932$

$-516.117351$

0.185126

0.196059

0.149614

$-515.921292$

$-515.967737$

0.181535

0.754

0.7539

C1

0

2.06343

1.1271

0.92935

1.170540

0.969420

$-515.972327$

$-515.930877$

$-515.966826$

$-515.924883$

$-516.019790$

$-516.013271$

$-515.971328$ 


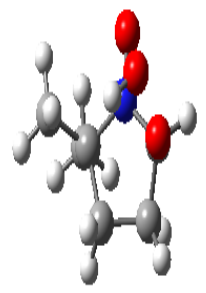

\begin{tabular}{lr} 
Compound & \multicolumn{2}{c}{ DMPO-O2H 22.5 deg } \\
file & oxdp_337_5.log \\
E 6-31+g** tight & -516.1529572 \\
E 6-31 g* & -516.110154 \\
ZPE (raw) & 0.184653 \\
Therm Corr to H (raw) & 0.195809 \\
Therm Corr to G (raw) & 0.149080 \\
H (raw) & -515.914345 \\
G (raw) & -515.961074 \\
ZPE (scaled) & 0.181071 \\
Electronic State & \\
s2 6-31 g* & 0.7534 \\
Symmetry & \\
N Imag & 1.889610 \\
Rotational Con. (GHz) & 1.185320 \\
Rotational Con. (GHz) & 1.031710 \\
Rotational Con. (GHz) & \\
H298 & \\
6-31+g** tight & -515.960730 \\
6-31 g* & -515.917928 \\
G298 & \\
6-31+g** tight & \\
6-31 g* & \\
\hline
\end{tabular}


Table S4

Thermodynamic data of the various conformations of the Conformation B DMPO- $\mathrm{O}_{2} \mathrm{H}$ at the B3LYP/6-31+G(d,p)//B3LYP/6-31G(d) level

(based on Figure 2) 
b3lyp/6-31+g**//b3lyp/6-31g*

ZPE scaling factor $=$
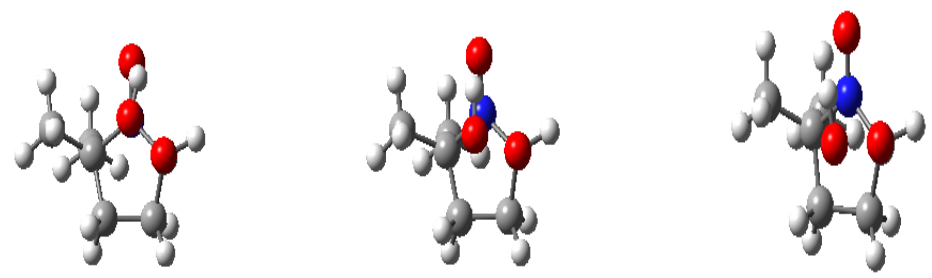

\section{Compound \\ file \\ E 6-31+g** tight \\ E 6-31g* \\ ZPE (raw) \\ H (raw) \\ G (raw) \\ ZPE (scaled) \\ Electronic State \\ s2 6-31g* \\ Symmetry \\ $N$ Imag \\ H298 \\ 6-31+g** tight \\ 6-31g* \\ G298 \\ $6-31+g^{* *}$ tight \\ 6-31g*}

Therm Corr to $\mathbf{H}$ (raw)

Therm Corr to G (raw)

Rotational Con. (GHz)

Rotational Con. (GHz)

Rotational Con. (GHz)

\section{DMPO-O2Hb 360 deg}

oxdp_0b.log

$-516.1563315$

$-516.1149029$

0.185234

0.196056

0.150211

$-515.918847$

$-515.964692$

0.181640

0.7538

1

DMPO-O2Hb 337.5 deg oxdp_22_5b.log

$-516.1589203$

$-516.117604$

0.185466

0.196195

0.150492

$-515.921409$

$-515.967112$

0.181868

0.7538

1

DMPO-O2Hb 315 deg oxdp_45b.log

$-516.1625021$

$-516.1206797$

0.185563

0.197185

0.149011

$-515.923494$

$-515.971669$

0.181963

0.7537

0
$-515.963869$

$-515.922440$

$-516.009714$

$-515.968285$
$-515.966323$

$-515.925007$

$-516.012026$

$-515.970710$
$-515.968917$

$-515.927095$

$-516.017091$

$-515.975269$ 


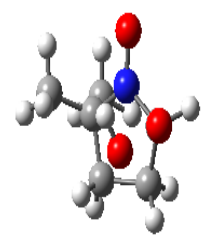

Compound
file
E 6-31+g** tight
E 6-31g*
ZPE (raw)
Therm Corr to H (raw)
Therm Corr to G (raw)
H (raw)
G (raw)
ZPE (scaled)
Electronic State
s2 6-31g*
Symmetry
N Imag
Rotational Con. (GHz)
Rotational Con. (GHz)
Rotational Con. (GHz)
H298
6-31+g** tight
6-31g*
G298
6-31+g** tight
6-31g*

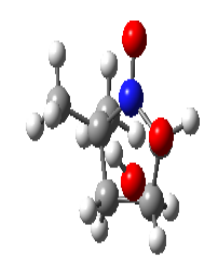

\begin{tabular}{|c|c|c|}
\hline $\begin{array}{l}\text { DMPO-O2Hb } 292.5 \text { deg } \\
\text { oxdp_67_5b.log }\end{array}$ & $\begin{array}{l}\text { DMPO-O2Hb } 270 \text { deg } \\
\text { oxdp_90b.log }\end{array}$ & $\begin{array}{l}\text { DMPO-O2Hb } 247.5 \mathrm{deg} \\
\text { oxdp_112.5b.log }\end{array}$ \\
\hline-516.164057 & -516.1608216 & -516.1555969 \\
\hline-516.1211923 & -516.1175307 & -516.112296 \\
\hline 0.185252 & 0.18515 & 0.1848 \\
\hline 0.197039 & 0.196989 & 0.195927 \\
\hline 0.148524 & 0.148251 & 0.149004 \\
\hline-515.924153 & -515.920541 & -515.916369 \\
\hline-515.972668 & -515.969279 & -515.963292 \\
\hline 0.181658 & 0.181558 & 0.181215 \\
\hline 0.7534 & 0.7534 & 0.7535 \\
\hline . & - & - \\
\hline 0 & 0 & \\
\hline - & - & - \\
\hline- & - & - \\
\hline - & - & - \\
\hline-515.970612 & -515.967424 & -515.963255 \\
\hline-515.927747 & -515.924134 & -515.919954 \\
\hline-516.019127 & -516.016162 & -516.010178 \\
\hline-515.976262 & -515.972872 & -515.966877 \\
\hline
\end{tabular}




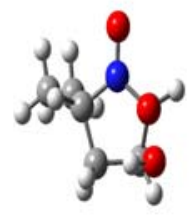

Compound
file
E 6-31+g** tight
E 6-31g*
ZPE (raw)
Therm Corr to H (raw)
Therm Corr to G (raw)
H (raw)
G (raw)
ZPE (scaled)
Electronic State
s2 6-31g*
Symmetry
N Imag
Rotational Con. (GHz)
Rotational Con. (GHz)
Rotational Con. (GHz)
H298
6-31+g
6-31g* tight
G298
6-31+g** tight
6-31g*

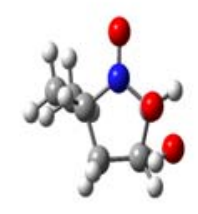

\begin{tabular}{|c|c|c|}
\hline $\begin{array}{l}\text { DMPO-O2Hb } 225 \text { deg } \\
\text { oxdp_135b.log }\end{array}$ & $\begin{array}{l}\text { DMPO-O2Hb } 202.5 \text { deg } \\
\text { oxdp_157.5b.log }\end{array}$ & $\begin{array}{l}\text { DMPO-O2Hb } 180 \mathrm{deg} \\
\text { oxdp_180b.log }\end{array}$ \\
\hline-516.1539661 & -516.1568716 & -516.1601804 \\
\hline-516.1108266 & -516.1137981 & -516.1168598 \\
\hline 0.184652 & 0.184751 & 0.184914 \\
\hline 0.195883 & 0.195923 & 0.196869 \\
\hline 0.148653 & 0.149041 & 0.148095 \\
\hline-515.914943 & -515.917875 & -515.919991 \\
\hline-515.962174 & -515.964758 & -515.968765 \\
\hline 0.181070 & 0.181167 & 0.181327 \\
\hline 0.7536 & 0.7536 & 0.7534 \\
\hline - & - & - \\
\hline 1 & 1 & 0 \\
\hline- & - & - \\
\hline - & - & - \\
\hline . & - & - \\
\hline-515.961665 & -515.964533 & -515.966899 \\
\hline-515.918526 & -515.921459 & -515.923578 \\
\hline-516.008895 & -516.011415 & -516.015673 \\
\hline-515.965756 & -515.968341 & -515.972352 \\
\hline
\end{tabular}




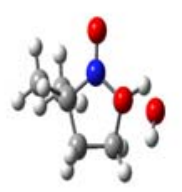

Compound
file
E 6-31+g** tight
E 6-31g*
ZPE (raw)
Therm Corr to H (raw)
Therm Corr to G (raw)
H (raw)
G (raw)
ZPE (scaled)
Electronic State
s2 6-31g*
Symmetry
N Imag
Rotational Con. (GHz)
Rotational Con. (GHz)
Rotational Con. (GHz)
H298
6-31+g** tight
6-31g*
G298
6-31+g** tight
6-31g*

oxdp_202.5b.log

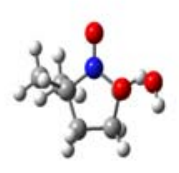

DMPO-O2Hb $157.5 \mathrm{deg}$

$-516.1602523$

$-516.1163819$

0.184819

0.19685

0.147602

$-515.919532$

$-515.96878$

0.181234

0.7533

0

DMPO-O2Hb 135 deg oxdp_225b.log $-516.1585535$

$-516.1145095$

0.184607

0.195791

0.14865

$-515.918718$

$-515.96586$

0.181026

0.7533

DMPO-O2Hb 112.5 deg

oxdp_247.5b.log

$-516.1583894$

$-516.1144311$

0.18457

0.195797

0.148507

$-515.918634$

$-515.965924$

0.180989

0.7534

1

1
$-515.966988$

$-515.923117$

$-516.016236$

$-515.972365$
$-515.966344$

$-515.922300$

$-516.013485$

$-515.969441$
$-515.966173$

$-515.922215$

$-516.013463$

$-515.969505$ 


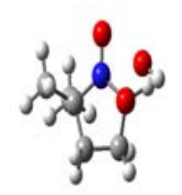

Compound
file
E 6-31+g** tight
E 6-31g*
ZPE (raw)
Therm Corr to H (raw)
Therm Corr to G (raw)
H (raw)
G (raw)
ZPE (scaled)
Electronic State
s2 6-31g*
Symmetry
N Imag
Rotational Con. (GHz)
Rotational Con. (GHz)
Rotational Con. (GHz)
H298
6-31+g** tight
6-31g*
G298
6-31+g** tight
6-31g*

\begin{tabular}{|c|c|c|}
\hline $\begin{array}{l}\text { DMPO-O2Hb } 90 \text { deg } \\
\text { oxdp_270b.log }\end{array}$ & $\begin{array}{l}\text { DMPO-O2Hb } 67.5 \mathrm{deg} \\
\text { oxdp_292.5b.log }\end{array}$ & $\begin{array}{l}\text { DMPO-O2Hb } 45 \text { deg } \\
\text { oxdp_315b.log }\end{array}$ \\
\hline-516.160225 & -516.1615978 & -516.1600823 \\
\hline-516.1165224 & -516.1181816 & -516.116908 \\
\hline 0.184817 & 0.184998 & 0.184919 \\
\hline 0.196898 & 0.196927 & 0.196865 \\
\hline 0.147057 & 0.148126 & 0.147790 \\
\hline-515.919625 & -515.921255 & -515.920043 \\
\hline-515.969466 & -515.970055 & -515.969117 \\
\hline 0.181232 & 0.181409 & 0.181332 \\
\hline 0.7534 & 0.7535 & 0.7535 \\
\hline - & - & - \\
\hline 0 & 0 & 0 \\
\hline - & - & - \\
\hline- & - & - \\
\hline - & - & - \\
\hline-515.966912 & -515.968260 & -515.966805 \\
\hline-515.923210 & -515.924844 & -515.923630 \\
\hline-516.016753 & -516.017061 & -516.015880 \\
\hline-515.973051 & -515.973645 & -515.972705 \\
\hline
\end{tabular}




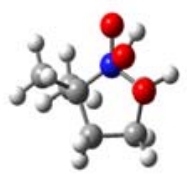

\begin{tabular}{|c|c|}
\hline $\begin{array}{l}\text { Compound } \\
\text { file }\end{array}$ & $\begin{array}{l}\text { DMPO-O2Hb } 22.5 \mathrm{deg} \\
\text { oxdp_337.5b.log }\end{array}$ \\
\hline E 6-31+g** tight & -516.1568417 \\
\hline E 6-31g* & -516.114731 \\
\hline ZPE (raw) & 0.184776 \\
\hline Therm Corr to $\mathrm{H}$ (raw) & 0.195937 \\
\hline Therm Corr to G (raw) & 0.149033 \\
\hline H (raw) & -515.918794 \\
\hline G (raw) & -515.965699 \\
\hline ZPE (scaled) & 0.181191 \\
\hline \multicolumn{2}{|l|}{ Electronic State } \\
\hline s2 6-31g* & 0.7536 \\
\hline Symmetry & - \\
\hline N Imag & 1 \\
\hline Rotational Con. (GHz) & - \\
\hline Rotational Con. (GHz) & - \\
\hline Rotational Con. (GHz) & - \\
\hline \multicolumn{2}{|l|}{$\mathrm{H} 298$} \\
\hline $6-31+g^{* \star}$ tight & -515.964489 \\
\hline $6-31 g^{*}$ & -515.922379 \\
\hline \multicolumn{2}{|l|}{ G298 } \\
\hline $6-31+g^{\star \star}$ tight & -516.011393 \\
\hline $6-31 g^{*}$ & -515.969283 \\
\hline
\end{tabular}


Table S5. Total energies (in hartrees), $\Delta E_{0, \text { rxn }}$ (in kcal/mol) ${ }^{\mathrm{a}}$ and Imaginary Vibrational Frequencies ${ }^{\mathrm{b}}$ of DMPO- $\mathrm{D}_{2}$, and Conformations A and $\mathrm{B}$ of of DMPO- ${ }_{2} \mathrm{H}$ at the PCM/B3LYP/6-31+G(d,p)//B3LYP/6-31G(d) level. (Based on Figure 3)

\begin{tabular}{|c|c|c|c|c|c|c|c|c|c|}
\hline \multirow{2}{*}{$\begin{array}{l}\text { Dihdral } \\
\text { angles }\end{array}$} & \multicolumn{3}{|c|}{ Total Energies (hartrees/part) } & \multicolumn{3}{|c|}{$\Delta E_{0, \text { rxn }}(\mathrm{kcal} / \mathrm{mol})$} & \multicolumn{3}{|c|}{$N_{\text {imag }}^{\mathrm{b}}$} \\
\hline & DMPO-O ${ }_{2}$ & DMPO-O ${ }_{2} \mathrm{H}$ A & DMPO- ${ }_{2} \mathrm{H} B$ & DMPO-O 2 & DMPO- ${ }_{2} \mathrm{H} A$ & DMPO- ${ }_{2} \mathrm{H} B$ & DMPO-O ${ }_{2}$ & $\begin{array}{r}\mathrm{DMPO} \\
\mathrm{A}\end{array}$ & $\begin{array}{l}\mathrm{O}-\mathrm{O}_{2} \mathrm{H} \\
\mathrm{B}\end{array}$ \\
\hline 0 & -515.674327 & -516.171268 & -516.171355 & 11.3 & -14.7 & -14.8 & 1 & 1 & 1 \\
\hline 337.5 & -515.675887 & -516.174486 & -516.160748 & 10.3 & -16.7 & -8.1 & 1 & 1 & 1 \\
\hline 315 & -515.684809 & -516.179441 & -516.16566 & 4.7 & -19.9 & -11.2 & 0 & 0 & 0 \\
\hline 292.5 & -515.683115 & -516.183185 & -516.169841 & 5.8 & -22.2 & -13.8 & 0 & 0 & 0 \\
\hline 270 & -515.681751 & -516.176431 & -516.167079 & 6.6 & -18.0 & -12.1 & 0 & 0 & 0 \\
\hline 247.5 & -515.676724 & -516.173317 & -516.162153 & 9.8 & -16.0 & -9.0 & 1 & 1 & 1 \\
\hline 225 & -515.679616 & -516.173108 & -516.160706 & 8.0 & -15.9 & -8.1 & 1 & 1 & 1 \\
\hline 202.5 & -515.682387 & -516.17824 & -516.163703 & 6.2 & -19.1 & -10.0 & 0 & 0 & 1 \\
\hline 180 & -515.690302 & -516.179744 & -516.167036 & 1.3 & -20.0 & -12.1 & 0 & 0 & 0 \\
\hline 157.5 & -515.68458 & -516.17873 & -516.1673 & 4.9 & -19.4 & -12.2 & 1 & 0 & 0 \\
\hline 135 & -515.684417 & -516.179414 & -516.165915 & 5.0 & -19.8 & -11.4 & 1 & 1 & 1 \\
\hline 112.5 & -515.683996 & -516.180095 & -516.165699 & 5.2 & -20.3 & -11.2 & 1 & 1 & 1 \\
\hline 90 & -515.690285 & -516.179991 & -516.167463 & 1.3 & -20.2 & -12.3 & 0 & 0 & 0 \\
\hline 67.5 & -515.681183 & -516.180849 & -516.168767 & 7.0 & -20.7 & -13.2 & 0 & 0 & 0 \\
\hline 45 & -515.68113 & -516.17418 & -516.16673 & 7.0 & -16.6 & -11.9 & 0 & 1 & 0 \\
\hline 22.5 & -515.674712 & -516.174311 & -516.160524 & 11.1 & -16.6 & -8.0 & 1 & 1 & 1 \\
\hline
\end{tabular}

${ }^{\mathrm{a}}$ Using the total energies (in hartrees) of DMPO $=-365.219175 ;{ }^{\bullet} \mathrm{O}_{2}=-150.473168 ; \cdot \mathrm{O}_{2} \mathrm{H}=-150.928629$. ${ }^{b} N$ imag refers to the number of imaginary vibrational frequencies $(1=\mathrm{TS})$. 


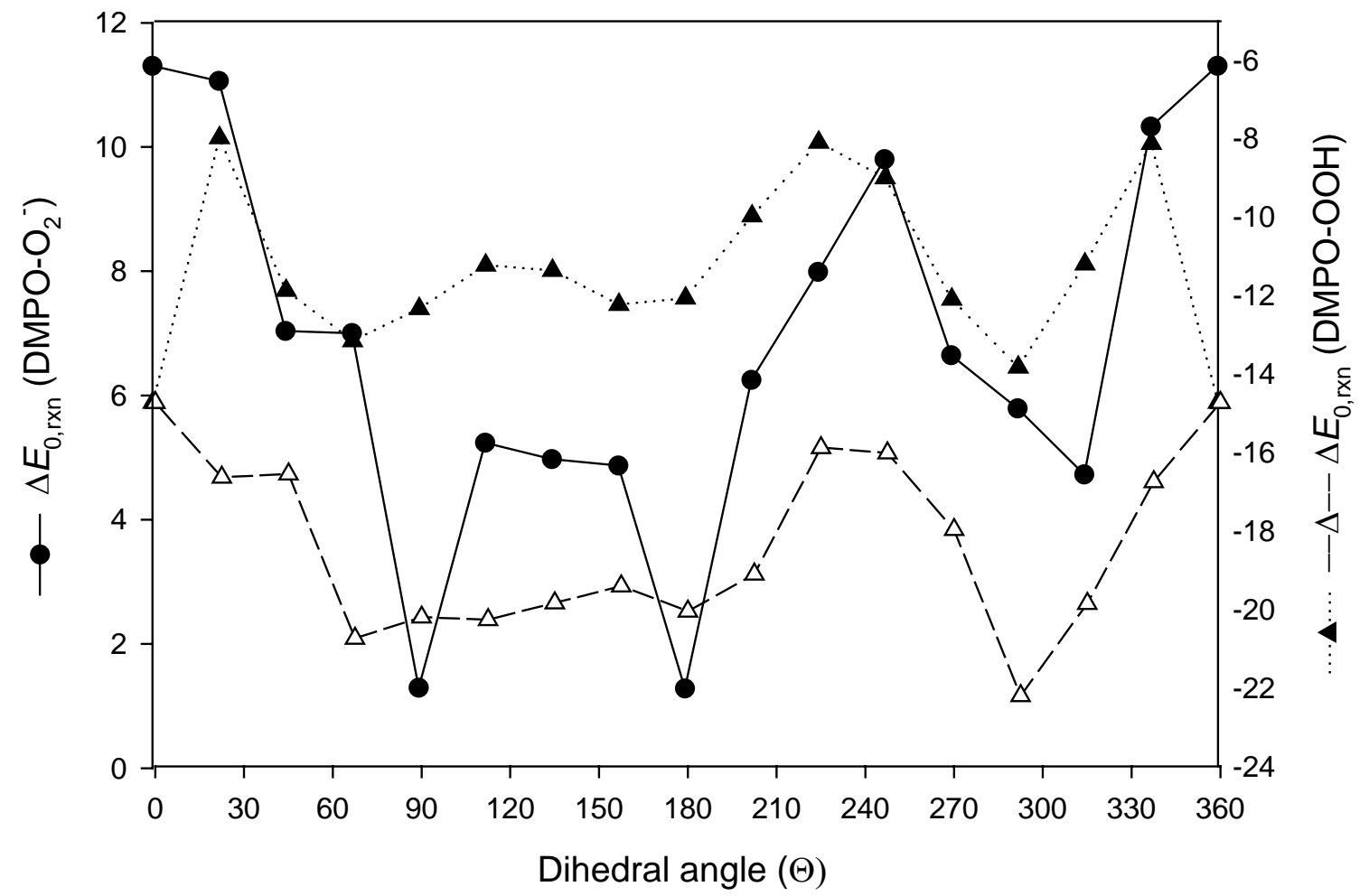

Figure S1. Rotational barriers in the DMPO- $\mathrm{O}_{2}^{-}(\bullet), \mathrm{DMPO}^{-} \mathrm{O}_{2} \mathrm{H}$ conformation $\mathrm{A}(\triangle)$ and DMPO- $\mathrm{O}_{2} \mathrm{H}$ conformation $\mathrm{B}(\boldsymbol{\Delta})$. The dihedral angles are at $22.5^{\circ}$ increments along the N-C-O-O angle as a function of the bottom-of-the-well energies $\left(\Delta E_{0, \mathrm{rxn}}\right)$ in $\mathrm{kcal} / \mathrm{mol}$ at the PCM B3LYP/6 31+G(d,p) //B3LYP/6-31G(d) level of theory using a dielectric constant for water. 


\section{Table S6}

Thermodynamic data of various acids at the PCM/B3LYP/6311+G(d)// B3LYP/6-311+G(d) level

(based on Table 2) 


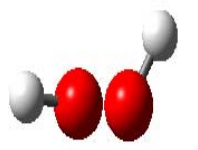

Compound
file
E 6-311+g* tight (PCM)
E 6-311+g*
ZPE (raw)
Therm Corr to H (raw)
Therm Corr to G (raw)
H (raw)
G (raw)
ZPE (scaled)
Electronic State
s2 6-31g*
Symmetry
N Imag
Rotational Con. (GHz)
Rotational Con. (GHz)
Rotational Con. (GHz)
H298
6-311+g* tight (PCM)
6-311+g*
G298
6-311+g* tight (PCM)
6-311+g*

\begin{tabular}{|c|c|c|}
\hline & Hydroperoxide ion Pagas & \multirow{2}{*}{368.7531463} \\
\hline $\mathrm{H} 2 \mathrm{O} 2 \_6311+\mathrm{Gd} . \log$ & HO2_6_311+Gd_b.log & \\
\hline-151.5981748 & -151.141177 $\Delta \Delta$ Gsolv & \\
\hline-151.589066 & -150.9881214 & -90.32774416 \\
\hline 0.026432 & 0.01287 & \\
\hline 0.030612 & 0.016752 & \\
\hline 0.004776 & -0.008928 & \\
\hline-151.558454 & -150.971369 & \\
\hline-151.58429 & -150.997049 & \\
\hline 0.025919 & 0.012620 & \\
\hline 0.000000 & 0.000000 & \\
\hline - & - & \\
\hline 0.000000 & 0.000000 & \\
\hline- & - & \\
\hline- & - & \\
\hline - & - & \\
\hline-151.568076 & -151.124674 & \\
\hline-151.558967 & -150.971619 & \\
\hline-151.593912 & -151.150354 & \\
\hline-151.584803 & -150.997299 & \\
\hline
\end{tabular}




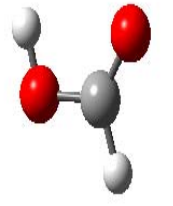

Compound
file
E 6-311+g* tight (PCM)
E 6-311+g*
ZPE (raw)
Therm Corr to H (raw)
Therm Corr to G (raw)
H (raw)
G (raw)
ZPE (scaled)
Electronic State
s2 6-31g*
Symmetry
N Imag
Rotational Con. (GHz)
Rotational Con. (GHz)
Rotational Con. (GHz)
H298
6-311+g* tight (PCM)
6-311+g*
G298
6-311+g* tight (PCM)
6-311+g*

\begin{tabular}{|c|c|c|}
\hline Formic Acid & Formate Ion & \\
\hline HCO2H_6311+Gd.log & HCO2_6311+Gd.log & 335.6253296 \\
\hline-189.8306726 & $-189.401467 \Delta \Delta$ Gsolv & \\
\hline-189.8198749 & -189.271540 & -74.75480199 \\
\hline 0.03372 & 0.019971 & \\
\hline 0.037828 & 0.023895 & \\
\hline 0.009643 & -0.003831 & \\
\hline-189.782047 & -189.247645 & \\
\hline-189.810232 & -189.275371 & \\
\hline 0.033066 & 0.019584 & \\
\hline 0 & 0.000000 & \\
\hline - & - & \\
\hline 0.000000 & 0.000000 & \\
\hline - & - & \\
\hline - & - & \\
\hline - & - & \\
\hline- & & \\
\hline-189.793499 & -189.377959 & \\
\hline-189.782701 & -189.248032 & \\
\hline-189.821684 & -189.405685 & \\
\hline-189.810886 & -189.275758 & \\
\hline
\end{tabular}




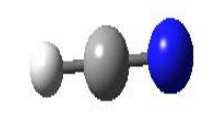

\begin{tabular}{|c|c|c|c|}
\hline Compound & Hydrocyanic acid & Cyanide Ion & \\
\hline $\begin{array}{l}\text { file } \\
\text { E 6-311+g* tight (PCM) }\end{array}$ & $\begin{array}{r}\text { HCN_6311+Gd.log } \\
-93.4552708\end{array}$ & $\begin{array}{r}\text { CN_6_311+Gd.log } \\
-93.01893 \Delta \Delta \text { Gsolv }\end{array}$ & 346.3703924 \\
\hline$E$ 6-311+g* & -93.45165511 & -92.88847739 & -79.59137614 \\
\hline ZPE (raw) & 0.016258 & 0.004835 & \\
\hline Therm Corr to $\mathrm{H}$ (raw) & 0.019753 & 0.00814 & \\
\hline Therm Corr to G (raw) & -0.003109 & -0.014204 & \\
\hline H (raw) & -93.431902 & -92.880338 & \\
\hline G (raw) & -93.454764 & -92.902682 & \\
\hline ZPE (scaled) & 0.015943 & 0.004741 & \\
\hline \multicolumn{4}{|l|}{ Electronic State } \\
\hline s2 6-31g* & 0.000000 & 0.000000 & \\
\hline Symmetry & - & - & \\
\hline N Imag & 0.000000 & 0.000000 & \\
\hline Rotational Con. (GHz) & - & - & \\
\hline Rotational Con. (GHz) & - & - & \\
\hline Rotational Con. (GHz) & - & - & \\
\hline \multicolumn{4}{|l|}{ H298 } \\
\hline $6-311+g^{*}$ tight $(\mathrm{PCM})$ & -93.435833 & -93.010884 & \\
\hline $6-311+g^{*}$ & -93.432218 & -92.880431 & \\
\hline \multicolumn{4}{|l|}{ G298 } \\
\hline $6-311+g^{*}$ tight $(\mathrm{PCM})$ & -93.458695 & -93.033228 & \\
\hline $6-311+g^{*}$ & -93.455080 & -92.902775 & \\
\hline
\end{tabular}




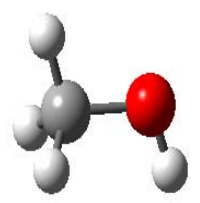

\begin{tabular}{|c|c|c|c|}
\hline $\begin{array}{l}\text { Compound } \\
\text { file }\end{array}$ & $\begin{array}{l}\text { Methanol } \\
\text { MeOH } 6311+\mathrm{Gd} \text { b log }\end{array}$ & $\begin{array}{l}\text { Methoxide ion } \\
\text { MeO } 6311+G d l o g\end{array}$ & 373.9057509 \\
\hline E 6-311+g* tight (PCM) & -115.7632471 & $-115.298393 \Delta \Delta$ Gsolv & \\
\hline$E$ 6-311+g* & -115.7538763 & -115.142076 & -92.20994384 \\
\hline ZPE (raw) & 0.051175 & 0.034916 & \\
\hline Therm Corr to $\mathrm{H}$ (raw) & 0.055445 & 0.038768 & \\
\hline Therm Corr to G (raw) & 0.028426 & 0.012675 & \\
\hline H (raw) & -115.698431 & -115.103308 & \\
\hline G (raw) & -115.72545 & -115.129401 & \\
\hline ZPE (scaled) & 0.050182 & 0.034239 & \\
\hline \multicolumn{4}{|l|}{ Electronic State } \\
\hline s2 6-31g* & 0.000000 & 0.000000 & \\
\hline Symmetry & - & - & \\
\hline N Imag & 0.000000 & 0.000000 & \\
\hline Rotational Con. (GHz) & - & - & \\
\hline Rotational Con. (GHz) & - & - & \\
\hline Rotational Con. (GHz) & - & - & \\
\hline \multicolumn{4}{|l|}{$\mathrm{H} 298$} \\
\hline 6-311+g* tight (PCM) & -115.708795 & -115.260302 & \\
\hline $6-311+g^{*}$ & -115.699424 & -115.103985 & \\
\hline \multicolumn{4}{|l|}{ G298 } \\
\hline $6-311+g^{*}$ tight $(\mathrm{PCM})$ & -115.735814 & -115.286395 & \\
\hline $6-311+g^{*}$ & -115.726443 & -115.130078 & \\
\hline
\end{tabular}




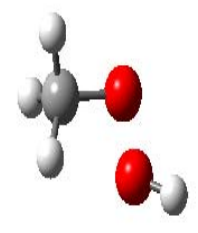

Compound

file

E 6-311+g* tight (PCM)

E 6-311+g*

ZPE (raw)

Therm Corr to $\mathrm{H}$ (raw)

Therm Corr to $\mathbf{G}$ (raw)

H (raw)

G (raw)

ZPE (scaled)

Electronic State

s2 6-31g*

Symmetry

N Imag

Rotational Con. (GHz)

Rotational Con. (GHz)

Rotational Con. (GHz)

H298

6-311+g* tight (PCM)

6-311+g*

G298

6-311+g* tight (PCM)

6-311+g*

\begin{tabular}{|c|c|c|}
\hline MethylO2H & MethylO2- & \\
\hline CH3O2H_6_311+Gd.log & CH3O2_6_311+Gd.log & 365.2880976 \\
\hline-190.9174364 & $-190.4612831 \Delta \Delta$ Gsolv & \\
\hline-190.909011 & -190.3136189 & -87.3736773 \\
\hline 0.054611 & 0.04108 & \\
\hline 0.059966 & 0.045762 & \\
\hline 0.028968 & 0.016268 & \\
\hline-190.849045 & -190.267857 & \\
\hline-190.880043 & -190.297351 & \\
\hline 0.053552 & 0.040283 & \\
\hline 0 & 0.000000 & \\
\hline - & - & \\
\hline 0.000000 & 0.000000 & \\
\hline- & - & \\
\hline - & - & \\
\hline - & - & \\
\hline-190.858530 & -190.416318 & \\
\hline-190.850104 & -190.268654 & \\
\hline-190.889528 & -190.445812 & \\
\hline-190.881102 & -190.298148 & \\
\hline
\end{tabular}




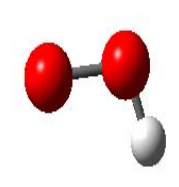

\begin{tabular}{|c|c|c|c|}
\hline $\begin{array}{l}\text { Compound } \\
\text { file }\end{array}$ & $\begin{array}{l}\text { Hydroperoxyl radical } \\
\text { peroxyl_6311+Gd.log }\end{array}$ & $\begin{array}{l}\text { Superoxide radical } \\
\text { o2_6311+Gd.log }\end{array}$ & 344.5111186 \\
\hline E 6-311+g* tight (PCM) & -150.9562463 & $-150.532358 \Delta \Delta$ Gsolv & \\
\hline$E$ 6-311+g* & -150.951952 & -150.3917078 & -85.56475002 \\
\hline ZPE (raw) & 0.014108 & 0.002655 & \\
\hline Therm Corr to $\mathrm{H}$ (raw) & 0.01791 & 0.005979 & \\
\hline Therm Corr to G (raw) & -0.008064 & -0.017138 & \\
\hline H (raw) & -150.934042 & -150.385729 & \\
\hline G (raw) & -150.960016 & -150.408846 & \\
\hline ZPE (scaled) & 0.013834 & 0.002603 & \\
\hline \multicolumn{4}{|l|}{ Electronic State } \\
\hline s2 6-31g* & 0.753800 & 0.756500 & \\
\hline Symmetry & - & - & \\
\hline N Imag & 0.000000 & 0.000000 & \\
\hline Rotational Con. (GHz) & - & - & \\
\hline Rotational Con. (GHz) & - & - & \\
\hline Rotational Con. (GHz) & - & - & \\
\hline \multicolumn{4}{|l|}{ H298 } \\
\hline $6-311+g^{*}$ tight $(\mathrm{PCM})$ & -150.938610 & -150.526431 & \\
\hline $6-311+g^{*}$ & -150.934316 & -150.385780 & \\
\hline \multicolumn{4}{|l|}{ G298 } \\
\hline $6-311+g^{*}$ tight $(\mathrm{PCM})$ & -150.964584 & -150.549548 & \\
\hline $6-311+g^{*}$ & -150.960290 & -150.408897 & \\
\hline
\end{tabular}
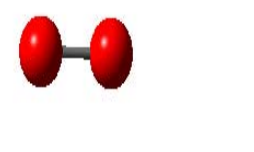

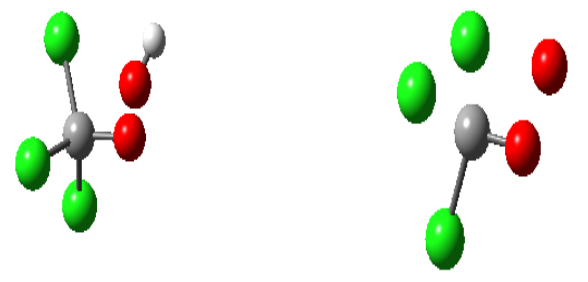

\begin{tabular}{|c|c|c|c|}
\hline $\begin{array}{l}\text { Compound } \\
\text { file }\end{array}$ & $\begin{array}{l}\text { сcl3coOH } \\
\text { CCl3O2H } 6311+\text { Gd.log }\end{array}$ & $\begin{array}{l}\text { ccl30o- } \\
\text { CCl3O2_6311+Gd.log }\end{array}$ & 330.6365718 \\
\hline E 6-311+g* tight (PCM) & -1569.767739 & $-1569.335387 \Delta \Delta$ Gsolv & \\
\hline E 6-311+g* & -1569.76236 & -1569.222439 & -67.50072001 \\
\hline ZPE (raw) & 0.026177 & 0.012901 & \\
\hline Therm Corr to $\mathrm{H}$ (raw) & 0.034012 & 0.020724 & \\
\hline Therm Corr to G (raw) & -0.0058 & -0.019316 & \\
\hline H (raw) & -1569.728348 & -1569.201715 & \\
\hline G (raw) & -1569.76816 & -1569.241755 & \\
\hline ZPE (scaled) & 0.025669 & 0.012651 & \\
\hline \multicolumn{4}{|l|}{ Electronic State } \\
\hline s2 6-31g* & 0 & 0 & \\
\hline Symmetry & - & - & \\
\hline N Imag & 0.000000 & 0.000000 & \\
\hline Rotational Con. (GHz) & - & 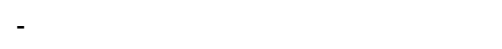 & \\
\hline Rotational Con. (GHz) & - & - & \\
\hline Rotational Con. (GHz) & - & - & \\
\hline \multicolumn{4}{|l|}{ H298 } \\
\hline $6-311+g^{*}$ tight $(\mathrm{PCM})$ & -1569.734235 & -1569.314913 & \\
\hline $6-311+g^{*}$ & -1569.728856 & -1569.201965 & \\
\hline \multicolumn{4}{|l|}{ G298 } \\
\hline $6-311+g^{*}$ tight $(\mathrm{PCM})$ & -1569.774047 & -1569.354953 & \\
\hline $6-311+g^{*}$ & -1569.768668 & -1569.242005 & \\
\hline
\end{tabular}




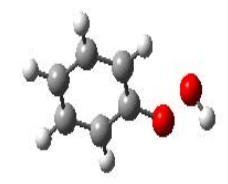

Compound

file

E 6-311+g* tight (PCM)

E 6-311+g*

ZPE (raw)

Therm Corr to $\mathbf{H}$ (raw)

Therm Corr to G (raw)

H (raw)

G (raw)

ZPE (scaled)

Electronic State

s2 6-31g*

Symmetry

N Imag

Rotational Con. (GHz)

Rotational Con. (GHz)

Rotational Con. (GHz)

H298

6-311+g* tight (PCM)

6-311+g*

G298

6-311+g* tight (PCM)

6-311+g*

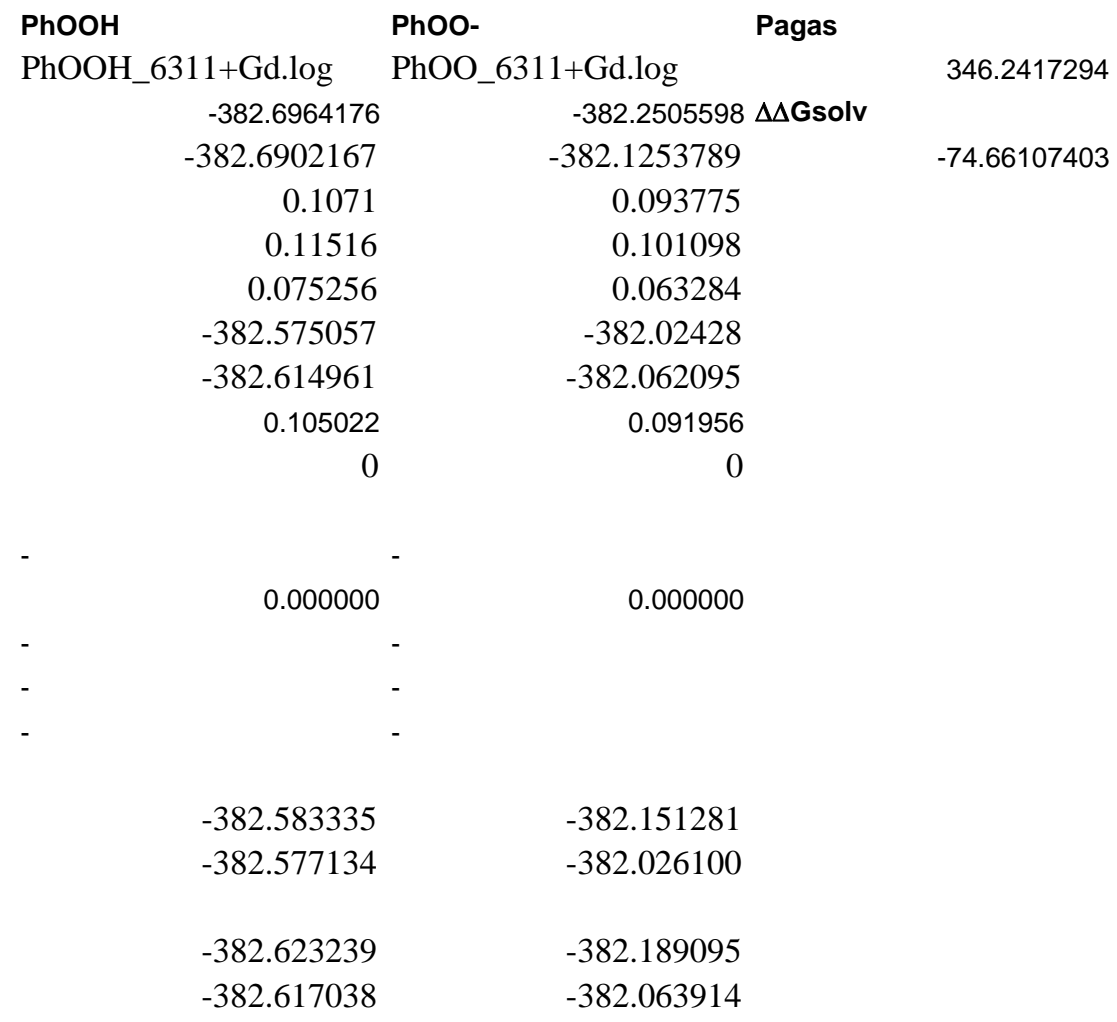




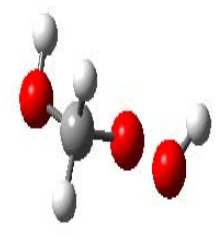

Compound
file
E 6-311+g* tight (PCM)
E 6-311+g*
ZPE (raw)
Therm Corr to H (raw)
Therm Corr to G (raw)
H (raw)
G (raw)
ZPE (scaled)
Electronic State
s2 6-31g*
Symmetry
N Imag
Rotational Con. (GHz)
Rotational Con. (GHz)
Rotational Con. (GHz)
H298
6-311+g* tight (PCM)
6-311+g*
G298
6-311+g* tight (PCM)
6-311+g*

\begin{tabular}{|c|c|c|}
\hline $\begin{array}{l}\text { OHCH2OOH } \\
\text { ohch2ooh } 6311+\mathrm{Gd} \text { log }\end{array}$ & OHCH2OO- & 356.8307444 \\
\hline-266.1637299 & -265.7035503 $\Delta \Delta$ Gsolv & \\
\hline-266.1491968 & -265.5672765 & -76.39347465 \\
\hline 0.059957 & 0.04642 & \\
\hline 0.066155 & 0.05212 & \\
\hline 0.032284 & 0.01932 & \\
\hline-266.083041 & -265.515156 & \\
\hline-266.116913 & -265.547957 & \\
\hline 0.058794 & 0.045519 & \\
\hline 0 & 0 & \\
\hline - & - & \\
\hline 0.000000 & 0.000000 & \\
\hline- & - & \\
\hline- & - & \\
\hline- & - & \\
\hline - & & \\
\hline-266.098738 & -265.652331 & \\
\hline-266.084205 & -265.516057 & \\
\hline-266.132609 & -265.685131 & \\
\hline-266.118076 & -265.548857 & \\
\hline
\end{tabular}

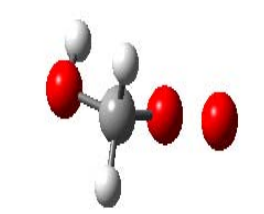




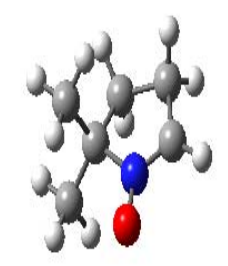

\begin{tabular}{|c|c|}
\hline Compound & DMPO \\
\hline file & dmpo_6_311+Gd.log \\
\hline E 6-311+g* tight (PCM) & -365.279311 \\
\hline$E$ 6-311+g* & -365.2687238 \\
\hline ZPE (raw) & 0.165591 \\
\hline Therm Corr to $\mathrm{H}$ (raw) & 0.174526 \\
\hline Therm Corr to G (raw) & 0.133777 \\
\hline H (raw) & -365.094198 \\
\hline G (raw) & -365.134947 \\
\hline ZPE (scaled) & 0.16559 \\
\hline \multicolumn{2}{|l|}{ Electronic State } \\
\hline s2 6-31g* & 0 \\
\hline Symmetry & - \\
\hline N Imag & 0.000000 \\
\hline Rotational Con. (GHz) & - \\
\hline Rotational Con. (GHz) & - \\
\hline Rotational Con. (GHz) & - \\
\hline \multicolumn{2}{|l|}{ H298 } \\
\hline $6-311+g^{*}$ tight $(\mathrm{PCM})$ & -365.104785 \\
\hline $6-311+g^{*}$ & -365.094198 \\
\hline \multicolumn{2}{|l|}{ G298 } \\
\hline $6-311+g^{*}$ tight $(\mathrm{PCM})$ & -365.145534 \\
\hline $6-311+g^{*}$ & -365.134947 \\
\hline
\end{tabular}




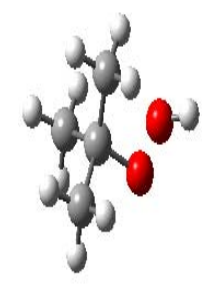

Compound

file

E 6-311+g* tight (PCM)

E 6-311+g*

ZPE (raw)

Therm Corr to $\mathrm{H}$ (raw)

Therm Corr to $\mathrm{G}$ (raw)

H (raw)

G (raw)

ZPE (scaled)

Electronic State

s2 6-31g*

Symmetry

N Imag

Rotational Con. (GHz)

Rotational Con. (GHz)

Rotational Con. (GHz)

H298

6-311+g* tight (PCM)

6-311+g*

G298

6-311+g* tight (PCM)

6-311+g*

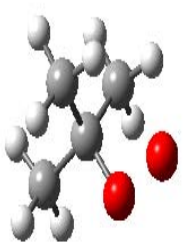

tBuoo-

Pagas

tbuO2H_6311+GdF.log tbuO2_6311+GdF.log -308.4377991 $\Delta \Delta$ Gsolv

$-308.293274$

0.124513

0.133063

0.093906

$-308.160211$

$-308.199368$

0.122097

0

0.000000

0.000000

$-308.753375$

$-308.307152$

$-308.741799$

$-308.162627$

$-308.793346$

$-308.346309$

$-308.781770$

$-308.201784$
363.7066051

$-83.42662497$ 


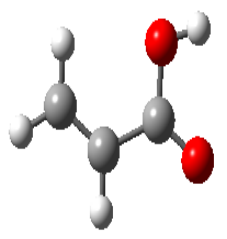

Compound
file
E 6-311+g* tight (PCM)
E 6-311+g*
ZPE (raw)
Therm Corr to H (raw)
Therm Corr to G (raw)
H (raw)
G (raw)
ZPE (scaled)
Electronic State
s2 6-31g*
Symmetry
N Imag
Rotational Con. (GHz)
Rotational Con. (GHz)
Rotational Con. (GHz)
H298
6-311+g* tight (PCM)
6-311+g*
G298
6-311+g* tight (PCM)
6-311+g*

Acrylic Acid

$-267.2540516$

$-267.16808$

7.20205

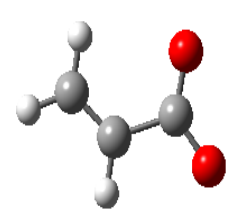

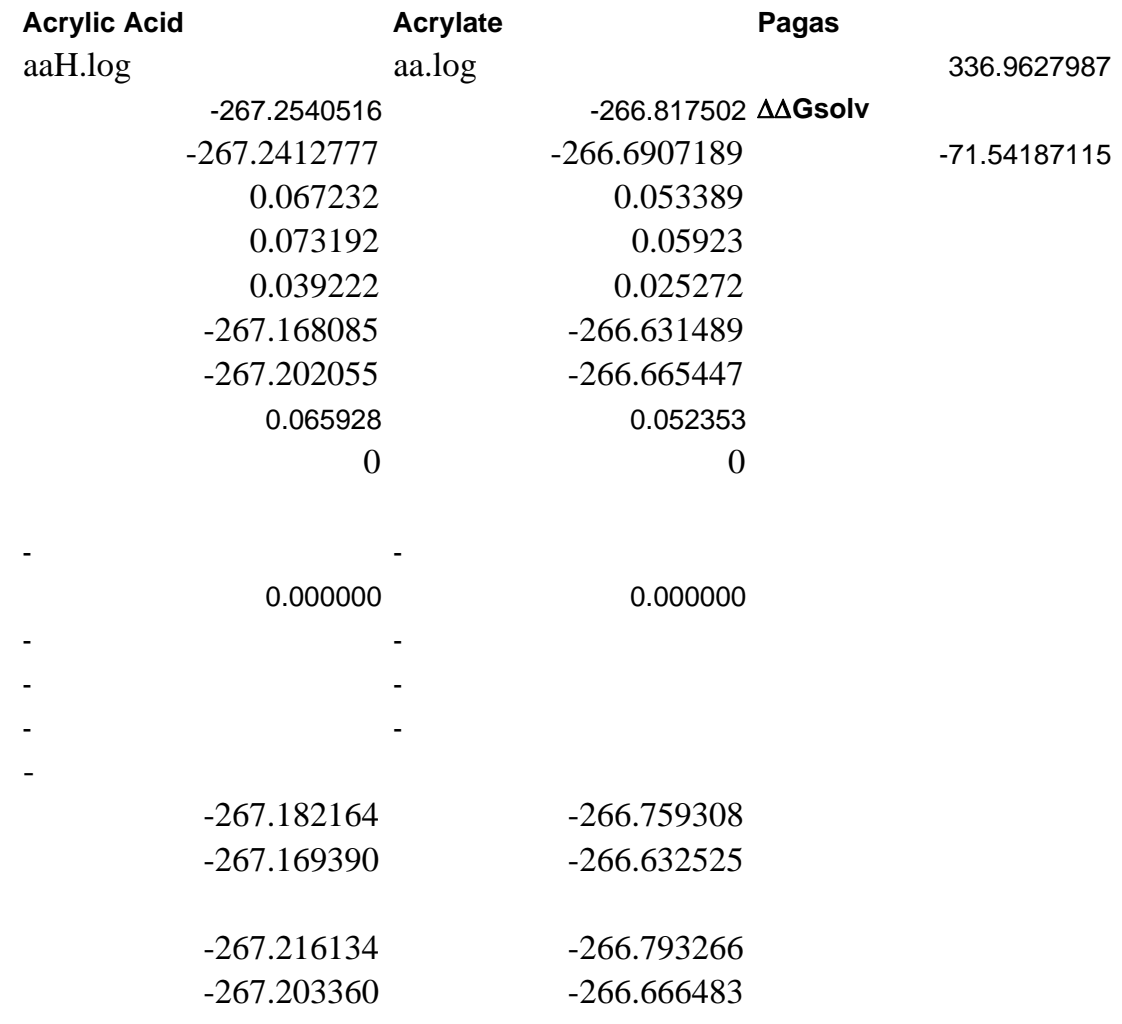



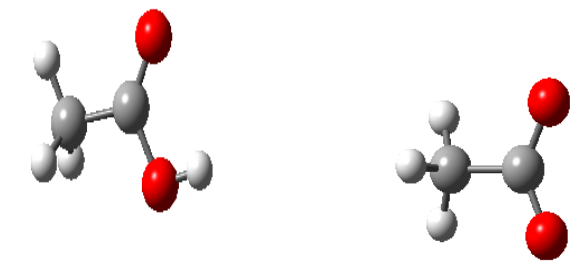

\begin{tabular}{|c|c|c|c|c|}
\hline Compound & Acetic acid & Acetate & Pagas & 340.606255 \\
\hline E 6-311+g* tight (PCM) & -229.1673053 & & -228.727967 $\Delta \Delta$ Gsolv & \\
\hline$E$ 6-311+g* & -229.1542163 & & -228.5979739 & -73.35845467 \\
\hline ZPE (raw) & 0.061641 & & 0.047923 & \\
\hline Therm Corr to $\mathrm{H}$ (raw) & 0.067192 & & 0.053328 & \\
\hline Therm Corr to G (raw) & 0.034138 & & 0.019988 & \\
\hline H (raw) & -229.087025 & & -228.544646 & \\
\hline G (raw) & -229.120078 & & -228.577985 & \\
\hline ZPE (scaled) & 0.060445 & & 0.046993 & \\
\hline Electronic State & 0 & & 0 & \\
\hline \multicolumn{5}{|l|}{ s2 6-31g* } \\
\hline Symmetry & - & - & & \\
\hline N Imag & 0.000000 & & 0.000000 & \\
\hline Rotational Con. (GHz) & - & - & & \\
\hline Rotational Con. (GHz) & - & - & & \\
\hline Rotational Con. (GHz) & - & - & & \\
\hline H298 & - & & & \\
\hline $6-311+g^{*}$ tight $(\mathrm{PCM})$ & -229.101309 & & -228.675569 & \\
\hline $6-311+g^{*}$ & -229.088220 & & -228.545576 & \\
\hline \multicolumn{5}{|l|}{ G298 } \\
\hline $6-311+g^{*}$ tight (PCM) & -229.134363 & & -228.708909 & \\
\hline $6-311+g^{*}$ & -229.121274 & & -228.578916 & \\
\hline
\end{tabular}



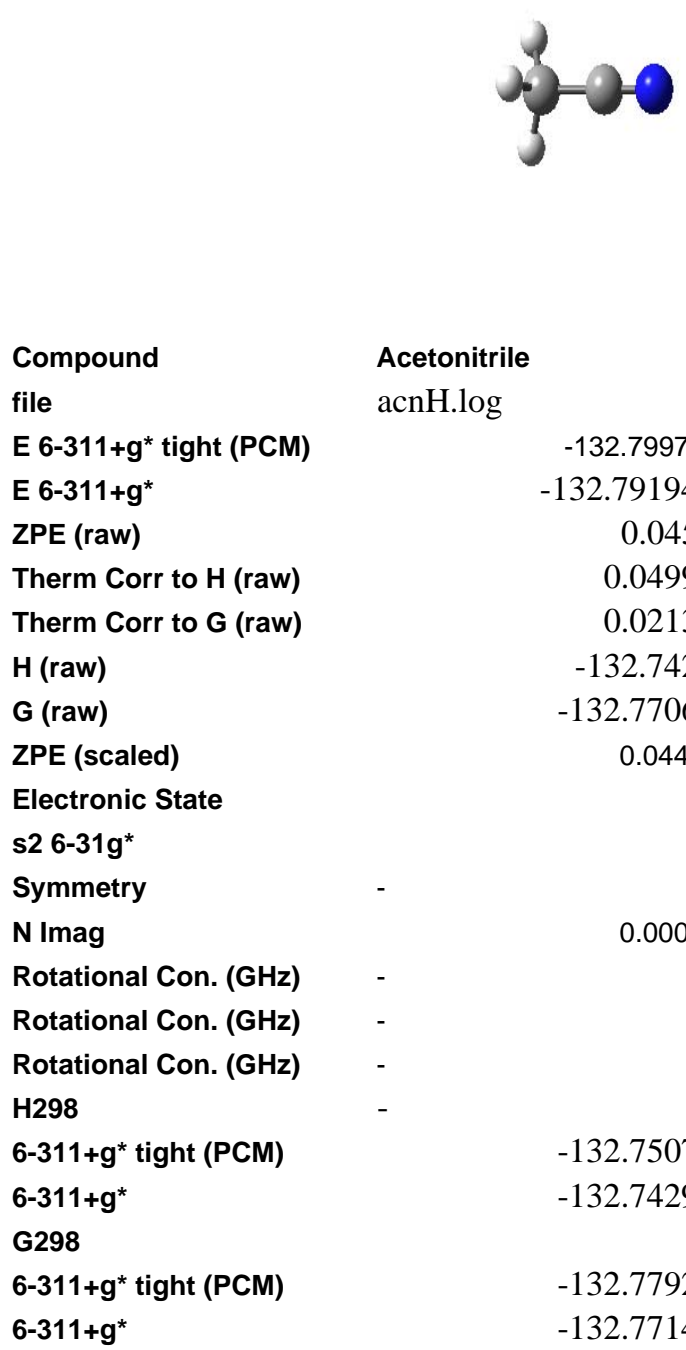

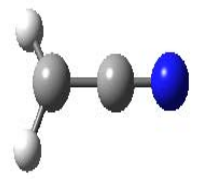

\begin{tabular}{|c|c|c|c|c|}
\hline $\begin{array}{l}\text { Acetonitrile } \\
\text { acnH.log }\end{array}$ & \multicolumn{2}{|c|}{$\begin{array}{l}\text { Acetonitrile anion } \\
\text { acn.log }\end{array}$} & Pagas & \multirow[t]{2}{*}{370.1703854} \\
\hline & -132.7997394 & -132.3066328 & $\Delta \Delta$ Gsolv & \\
\hline & -132.7919414 & -132.1872008 & & -70.0513403 \\
\hline & 0.04535 & 0.03022 & & \\
\hline & 0.049902 & 0.034919 & & \\
\hline & 0.021336 & 0.006497 & & \\
\hline & -132.74204 & -132.152282 & & \\
\hline & -132.770605 & -132.180704 & & \\
\hline & 0.044470 & 0.029634 & & \\
\hline & 0 & 0 & & \\
\hline- & & & & \\
\hline & 0.000000 & 0.000000 & & \\
\hline- & & & & \\
\hline- & & & & \\
\hline- & & & & \\
\hline- & & & & \\
\hline & -132.750717 & -132.272300 & & \\
\hline & -132.742919 & -132.152868 & & \\
\hline & -132.779283 & -132.300722 & & \\
\hline & -132.771485 & -132.181290 & & \\
\hline
\end{tabular}




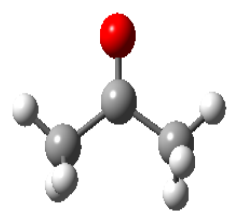

Compound
file
E 6-311+g* tight (PCM)
E 6-311+g*
ZPE (raw)
Therm Corr to H (raw)
Therm Corr to G (raw)
H (raw)
G (raw)
ZPE (scaled)
Electronic State
s2 6-31g*
Symmetry
N Imag
Rotational Con. (GHz)
Rotational Con. (GHz)
Rotational Con. (GHz)
H298
6-311+g* tight (PCM)
6-311+g*
G298
6-311+g* tight (PCM)
6-311+g*

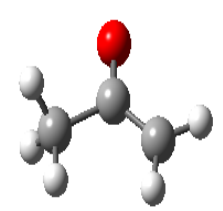

\begin{tabular}{|c|c|c|}
\hline $\begin{array}{l}\text { Acetone } \\
\text { actoneH.log }\end{array}$ & $\begin{array}{l}\text { Acetone anion } \\
\text { actone.log }\end{array}$ & 366.8046642 \\
\hline-193.2215993 & $-192.7401767 \Delta \Delta$ Gsolv & \\
\hline-193.2097274 & -192.6118361 & -73.08522072 \\
\hline 0.083587 & 0.069972 & \\
\hline 0.089879 & 0.075601 & \\
\hline 0.055546 & 0.043305 & \\
\hline-193.119849 & -192.536235 & \\
\hline-193.154181 & -192.568531 & \\
\hline 0.081965 & 0.068615 & \\
\hline 0 & 0 & \\
\hline- & - & \\
\hline- & 0.000000 & \\
\hline- & - & \\
\hline- & - & \\
\hline- & - & \\
\hline \multicolumn{3}{|l|}{ _ } \\
\hline -193.133342 & -192.665933 & \\
\hline-193.121470 & -192.537593 & \\
\hline-193.167675 & -192.698229 & \\
\hline-193.155803 & -192.569889 & \\
\hline
\end{tabular}



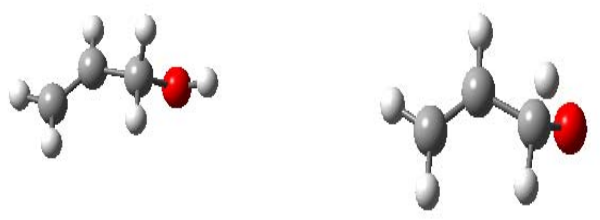

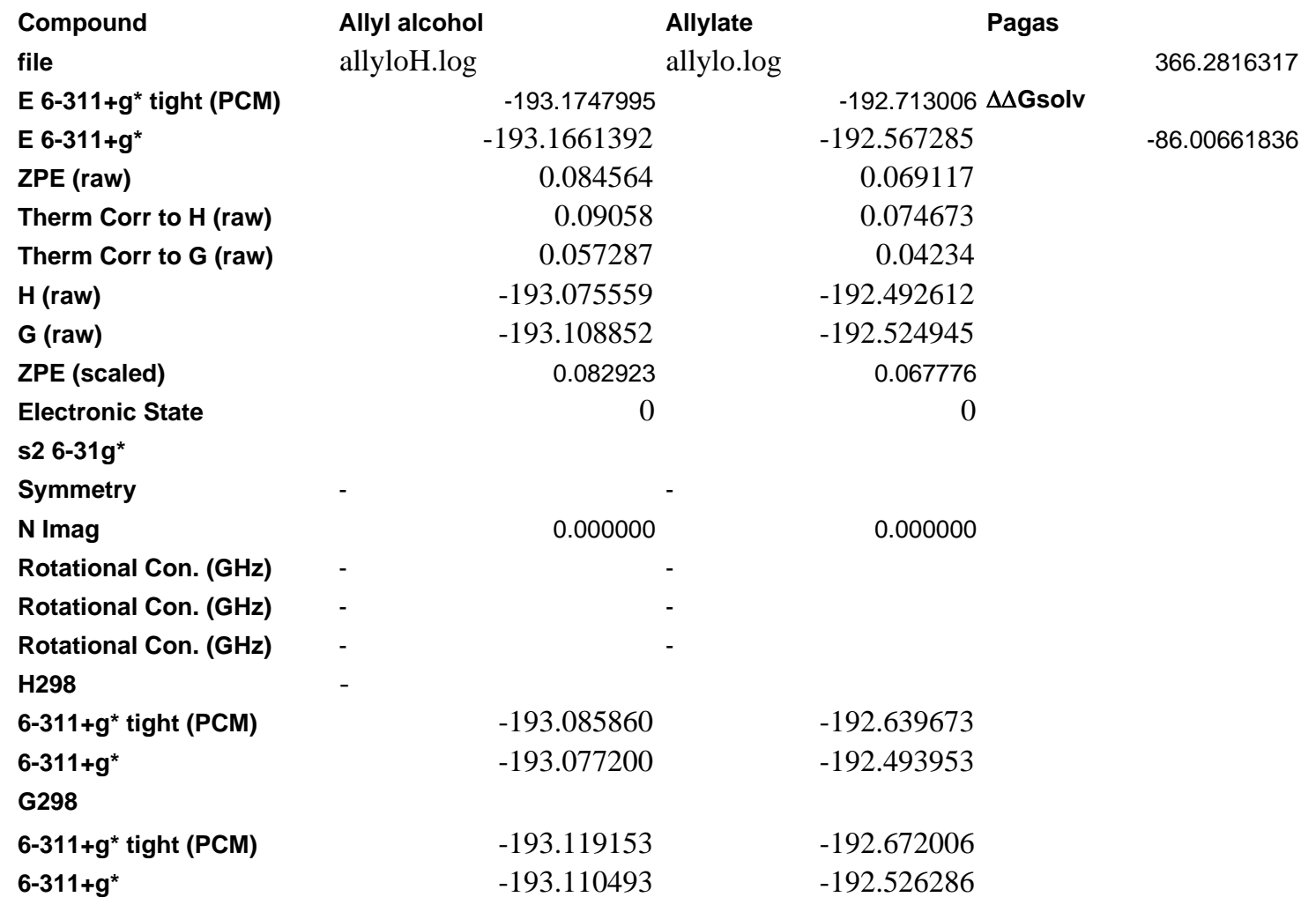



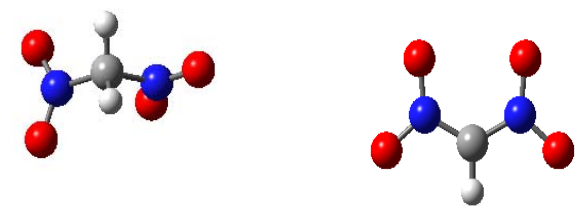

\begin{tabular}{|c|c|c|c|c|}
\hline $\begin{array}{l}\text { Compound } \\
\text { file }\end{array}$ & $\begin{array}{l}\text { Dinitromethane } \\
\text { dnmH.log }\end{array}$ & $\begin{array}{l}\text { Dinitrimethane anion } \\
\text { dnm.log }\end{array}$ & Pagas & 314.877055 \\
\hline E 6-311+g* tight (PCM) & -449.647763 & -449.2186115 & $\Delta \Delta$ Gsolv & \\
\hline$E$ 6-311+g* & -449.6309413 & -449.11605 & & -53.80252899 \\
\hline ZPE (raw) & 0.052863 & 0.039501 & & \\
\hline Therm Corr to $\mathrm{H}$ (raw) & 0.060228 & 0.046401 & & \\
\hline Therm Corr to G (raw) & 0.019749 & 0.008813 & & \\
\hline H (raw) & -449.570713 & -449.069649 & & \\
\hline G (raw) & -449.611192 & -449.107237 & & \\
\hline ZPE (scaled) & 0.051837 & 0.038735 & & \\
\hline Electronic State & 0 & 0 & ) & \\
\hline \multicolumn{5}{|l|}{ s2 6-31g* } \\
\hline Symmetry & - & - & & \\
\hline N Imag & 0.000000 & 0.000000 & & \\
\hline Rotational Con. (GHz) & - & - & & \\
\hline Rotational Con. (GHz) & - & - & & \\
\hline Rotational Con. (GHz) & - & - & & \\
\hline H298 & - & & & \\
\hline $6-311+g^{*}$ tight $(\mathrm{PCM})$ & -449.588561 & -449.172977 & & \\
\hline $6-311+g^{*}$ & -449.571739 & -449.070415 & & \\
\hline \multicolumn{5}{|l|}{ G298 } \\
\hline $6-311+g^{*}$ tight $(\mathrm{PCM})$ & -449.629040 & -449.210565 & & \\
\hline $6-311+g^{*}$ & -449.612218 & -449.108003 & & \\
\hline
\end{tabular}




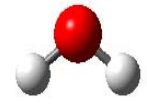

Compound
file
E 6-311+g* tight (PCM)
E 6-311+g*
ZPE (raw)
Therm Corr to H (raw)
Therm Corr to G (raw)
H (raw)
G (raw)
ZPE (scaled)
Electronic State
s2 6-31g*
Symmetry
N Imag
Rotational Con. (GHz)
Rotational Con. (GHz)
Rotational Con. (GHz)
H298
6-311+g* tight (PCM)
6-311+g*
G298
6-311+g* tight (PCM)
6-311+g*

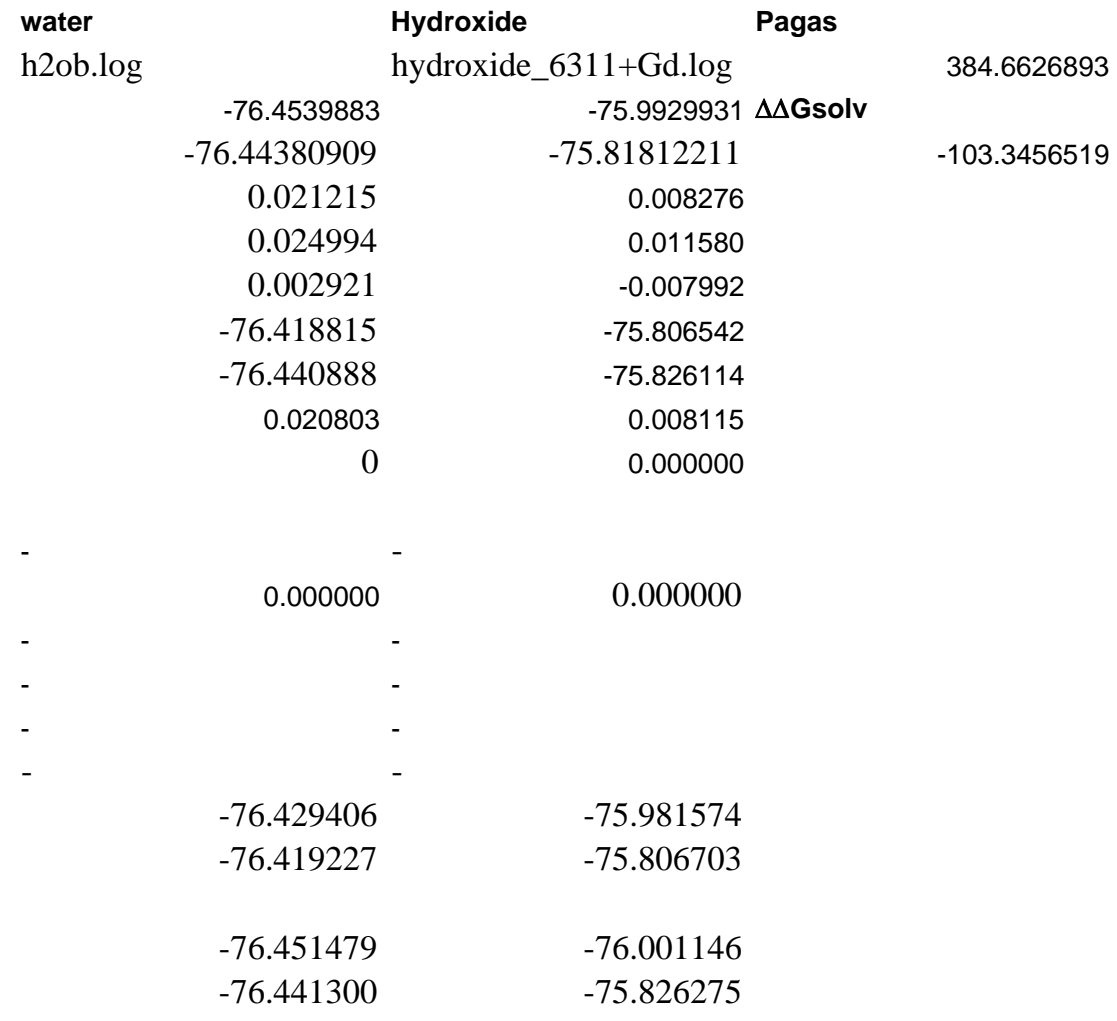




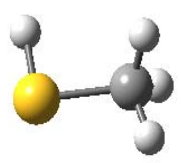

Compound
file
E 6-311+g* tight (PCM)
E 6-311+g*
ZPE (raw)
Therm Corr to H (raw)
Therm Corr to G (raw)
H (raw)
G (raw)
ZPE (scaled)
Electronic State
s2 6-31g*
Symmetry
N Imag
Rotational Con. (GHz)
Rotational Con. (GHz)
Rotational Con. (GHz)
H298
6-311+g* tight (PCM)
6-311+g*
G298
6-311+g* tight (PCM)
6-311+g*

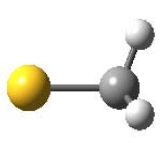

\begin{tabular}{|c|c|c|c|c|}
\hline $\begin{array}{l}\text { Methythiol } \\
\text { mesH.log }\end{array}$ & \multicolumn{2}{|r|}{$\begin{array}{l}\text { Methiolate } \\
\text { mes.log }\end{array}$} & Pagas & \multirow[t]{2}{*}{351.9806369} \\
\hline & -438.7355891 & & $-438.2841786 \Delta \Delta$ Gsolv & \\
\hline & -438.7344313 & & -438.163677 & -74.88930865 \\
\hline & 0.045967 & & 0.035935 & \\
\hline & 0.050509 & & 0.039946 & \\
\hline & 0.021737 & & 0.012315 & \\
\hline & -438.683922 & & -438.123731 & \\
\hline & -438.712694 & & -438.151362 & \\
\hline & 0.045075 & & 0.035238 & \\
\hline & 0 & & 0 & \\
\hline- & & - & & \\
\hline & 0.000000 & & 0.000000 & \\
\hline- & & - & & \\
\hline- & & - & & \\
\hline- & & - & & \\
\hline- & & & & \\
\hline & -438.685972 & & -438.244930 & \\
\hline & -438.684814 & & -438.124428 & \\
\hline & -438.714744 & & -438.272561 & \\
\hline & -438.713586 & & -438.152059 & \\
\hline
\end{tabular}




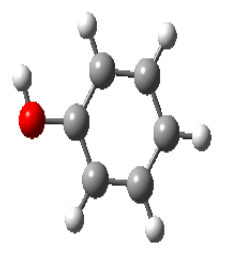

\begin{tabular}{|c|c|c|c|}
\hline $\begin{array}{l}\text { Compound } \\
\text { file }\end{array}$ & $\begin{array}{l}\text { Phenol } \\
\text { phenolH.log }\end{array}$ & $\begin{array}{l}\text { Phenolate } \\
\text { phenol.log }\end{array}$ & 341.5120857 \\
\hline E 6-311+g* tight (PCM) & -307.548979 & -307.1030998 $\Delta \Delta$ Gsolv & \\
\hline$E$ 6-311+g* & -307.5429436 & -306.9848016 & -70.44595969 \\
\hline ZPE (raw) & 0.104089 & 0.089906 & \\
\hline Therm Corr to $\mathrm{H}$ (raw) & 0.110613 & 0.096159 & \\
\hline Therm Corr to G (raw) & 0.075052 & 0.061019 & \\
\hline H (raw) & -307.43233 & -306.888643 & \\
\hline G (raw) & -307.467892 & -306.923782 & \\
\hline ZPE (scaled) & 0.102070 & 0.088162 & \\
\hline Electronic State & 0 & 0 & \\
\hline \multicolumn{4}{|l|}{ s2 6-31g* } \\
\hline Symmetry & - & - & \\
\hline N Imag & 0.000000 & 0.000000 & \\
\hline Rotational Con. (GHz) & - & - & \\
\hline Rotational Con. (GHz) & - & - & \\
\hline Rotational Con. (GHz) & - & - & \\
\hline H298 & - & & \\
\hline 6-311+g* tight (PCM) & -307.440385 & -307.008685 & \\
\hline $6-311+g^{*}$ & -307.434350 & -306.890387 & \\
\hline \multicolumn{4}{|l|}{ G298 } \\
\hline 6-311+g* tight (PCM) & -307.475946 & -307.043825 & \\
\hline $6-311+g^{*}$ & -307.469911 & -306.925527 & \\
\hline
\end{tabular}

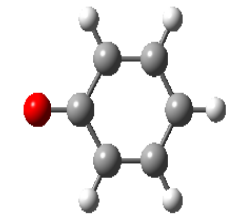




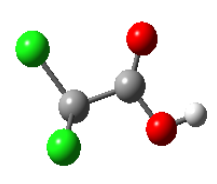

\begin{tabular}{|c|c|c|c|c|}
\hline Compound & Trichloroacetic acid & Trichloroacetate & Pagas & \\
\hline file & tcaH.log & tca.log & & 312.6682117 \\
\hline E 6-311+g* tight (PCM) & -1607.998347 & -1607.582056 & $\Delta \Delta$ Gsolv & \\
\hline$E$ 6-311+g* & -1607.987369 & -1607.475701 & & -59.85046931 \\
\hline ZPE (raw) & 0.033439 & 0.019774 & & \\
\hline Therm Corr to $\mathrm{H}$ (raw) & 0.041803 & 0.028243 & & \\
\hline Therm Corr to G (raw) & -0.000175 & -0.01458 & & \\
\hline H (raw) & -1607.945566 & -1607.447458 & & \\
\hline G (raw) & -1607.987544 & -1607.490281 & & \\
\hline ZPE (scaled) & 0.032790 & 0.019390 & & \\
\hline Electronic State & 0 & 0 & ) & \\
\hline \multicolumn{5}{|l|}{ s2 6-31g* } \\
\hline Symmetry & - & - & & \\
\hline N Imag & 0.000000 & 0.000000 & & \\
\hline Rotational Con. (GHz) & - & - & & \\
\hline Rotational Con. (GHz) & - & - & & \\
\hline Rotational Con. (GHz) & - & - & & \\
\hline H298 & - & & & \\
\hline $6-311+g^{*}$ tight $(\mathrm{PCM})$ & -1607.957192 & -1607.554197 & & \\
\hline $6-311+g^{*}$ & -1607.946215 & -1607.447841 & & \\
\hline \multicolumn{5}{|l|}{ G298 } \\
\hline $6-311+g^{*}$ tight (PCM) & -1607.999170 & -1607.597020 & & \\
\hline $6-311+g^{*}$ & -1607.988193 & -1607.490664 & & \\
\hline
\end{tabular}




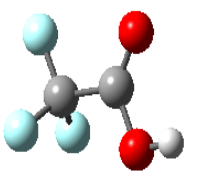

Compound
file
E 6-311+g* tight (PCM)
E 6-311+g*
ZPE (raw)
Therm Corr to H (raw)
Therm Corr to G (raw)
H (raw)
G (raw)
ZPE (scaled)
Electronic State
s2 6-31g*
Symmetry
N Imag
Rotational Con. (GHz)
Rotational Con. (GHz)
Rotational Con. (GHz)
H298
$6-311+g^{*}$ tight (PCM)
6-311+g*
G298
6-311+g* tight (PCM)
$6-311+g^{*}$

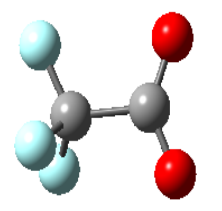

\begin{tabular}{|c|c|c|}
\hline $\begin{array}{l}\text { Triflouroactic acid } \\
\text { tfaH.log }\end{array}$ & $\begin{array}{l}\text { Triflouroacetate } \\
\text { tfab.log }\end{array}$ & 314.6141591 \\
\hline-526.9701495 & $-526.5527791 \Delta \Delta$ Gsolv & \\
\hline-526.9592397 & -526.4447556 & -60.93976933 \\
\hline 0.038644 & 0.02527 & \\
\hline 0.045829 & 0.032336 & \\
\hline 0.007271 & -0.006791 & \\
\hline-526.913411 & -526.41242 & \\
\hline-526.951969 & -526.451547 & \\
\hline 0.037894 & 0.024780 & \\
\hline 0 & 0 & \\
\hline - & - & \\
\hline 0.000000 & 0.000000 & \\
\hline- & - & \\
\hline- & - & \\
\hline- & - & \\
\hline - & & \\
\hline-526.925070 & -526.520933 & \\
\hline-526.914160 & -526.412910 & \\
\hline-6.846021968 & -67.7857913 & \\
\hline-526.963628 & -526.560060 & \\
\hline-526.952718 & -526.452037 & \\
\hline
\end{tabular}




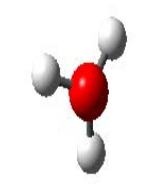

\begin{tabular}{|c|}
\hline $\begin{array}{l}\text { Compound } \\
\text { file }\end{array}$ \\
\hline $6-311+\mathrm{g}^{*}$ tight $(\mathrm{PCM}$ \\
\hline E 6-311+g* \\
\hline ZPE (raw) \\
\hline Therm Corr to $\mathrm{H}$ (raw) \\
\hline Therm Corr to G (raw) \\
\hline H (raw) \\
\hline G (raw) \\
\hline ZPE (scaled) \\
\hline Electronic State \\
\hline s2 6-31g* \\
\hline Symmetry \\
\hline N Imag \\
\hline Rotational Con. (GHz) \\
\hline Rotational Con. (GHz) \\
\hline Rotational Con. (GHz) \\
\hline H298 \\
\hline $6-311+g^{*}$ tight $(\mathrm{PCM})$ \\
\hline $6-311+g^{*}$ \\
\hline G298 \\
\hline $6-311+g^{*}$ tight $(P C M)$ \\
\hline $6-311+g^{*}$ \\
\hline
\end{tabular}

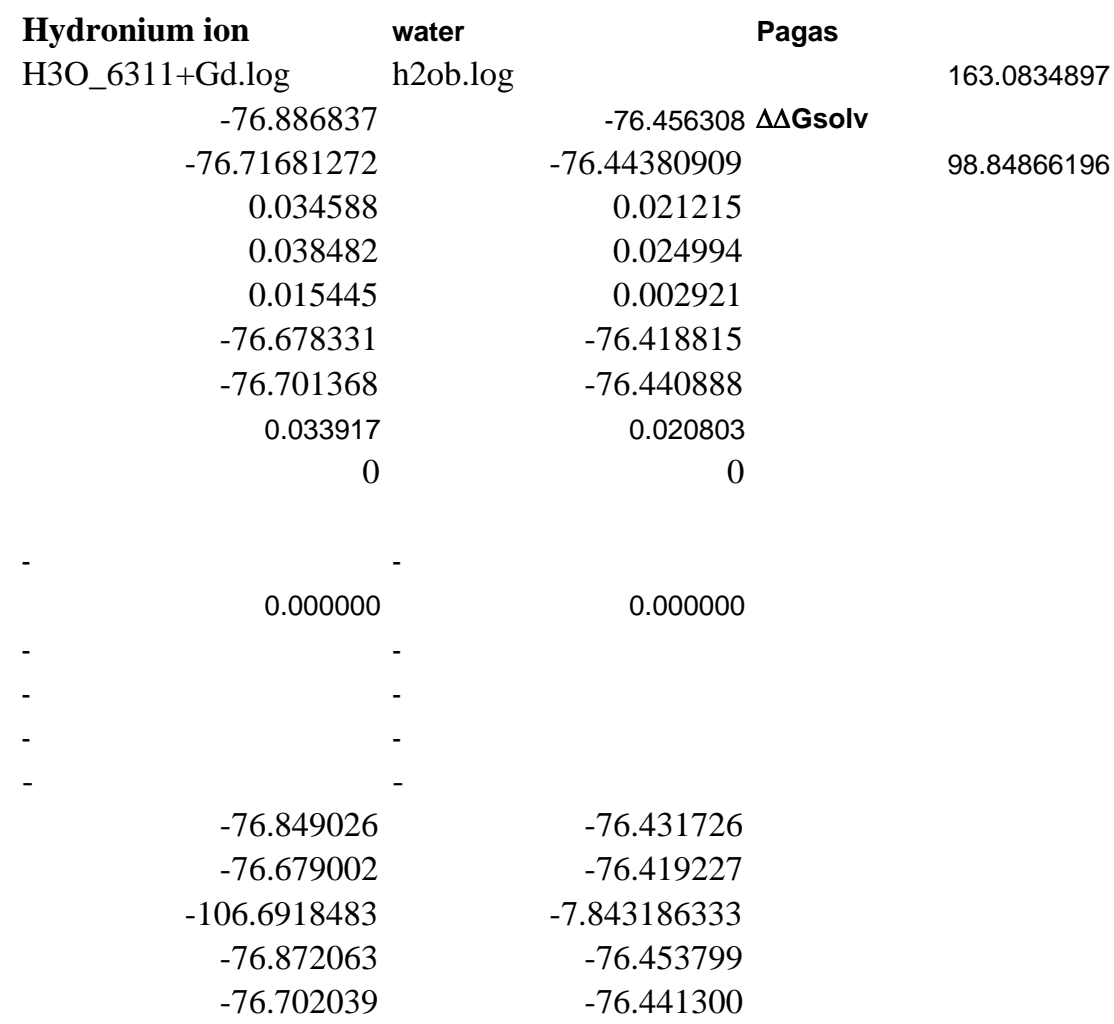




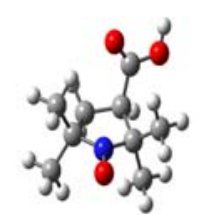

Compound

file

E 6-311+g* tight (PCM)

E 6-311+g*

ZPE (raw)

Therm Corr to $\mathrm{H}$ (raw)

Therm Corr to G (raw)

H (raw)

G (raw)

ZPE (scaled)

Electronic State

s2 6-31g*

Symmetry

N Imag

Rotational Con. (GHz)

Rotational Con. (GHz)

Rotational Con. (GHz)

H298

6-311+g* tight (PCM)

$6-311+g^{*}$

G298

6-311+g* tight (PCM)

6-311+g*

\begin{tabular}{|c|c|c|c|}
\hline СРН & CP- & Pagas & \\
\hline CPH_6311+Gd_2.log & CP_6311+Gd_2.log & & 329.2203686 \\
\hline-633.1724851 & -632.7287528 & $\Delta \Delta$ Gsolv & \\
\hline-633.1382382 & -632.6000255 & & -59.28731342 \\
\hline 0.247338 & 0.233503 & & \\
\hline 0.262321 & 0.248312 & & \\
\hline 0.206943 & 0.192493 & & \\
\hline-632.875917 & -632.351714 & & \\
\hline-632.931296 & -632.407533 & & \\
\hline 0.242540 & 0.228973 & & \\
\hline 0.7544 & 0.7546 & & \\
\hline C1 & C1 & & \\
\hline 0 & 0 & & \\
\hline- & - & & \\
\hline - & - & & \\
\hline- & - & & \\
\hline-632.914962 & -632.484971 & & \\
\hline-632.880716 & -632.356243 & & \\
\hline-21.49028208 & -80.7775955 & & \\
\hline-632.970340 & -632.540790 & & \\
\hline-632.936094 & -632.412062 & & \\
\hline
\end{tabular}



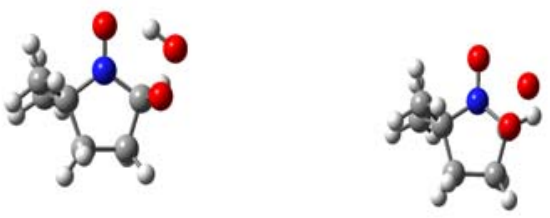

\begin{tabular}{|c|c|c|}
\hline DMPO-OOH adduct & DMPO-O2 90 PCM & 357.5698544 \\
\hline 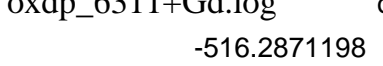 & $\begin{array}{l}\text { 02ap_90_0311+gapcm.log } \\
-515.8222688 \Delta \Delta \text { Gsolv }\end{array}$ & 351.5090344 \\
\hline-516.2622417 & -515.6780364 & -74.89596088 \\
\hline 0.184511 & 0.169845 & \\
\hline 0.196096 & 0.18129 & \\
\hline 0.148189 & 0.133473 & \\
\hline-516.066146 & -515.496746 & \\
\hline-516.114053 & -515.544563 & \\
\hline 0.180931 & 0.166550 & \\
\hline 0.7546 & 0.7571 & \\
\hline C1 & - & \\
\hline 0.000000 & 0 & \\
\hline 2.072340 & & \\
\hline 1.112730 & & \\
\hline 0.92072 & & \\
\hline-516.094603 & -515.644274 & \\
\hline-516.069725 & -515.500041 & \\
\hline & -90.50718489 & \\
\hline-516.142510 & -515.692091 & \\
\hline-516.117632 & -515.547858 & \\
\hline
\end{tabular}




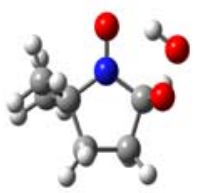

\begin{tabular}{|c|c|c|c|}
\hline $\begin{array}{l}\text { DMPO-OOH adduct } \\
\text { oxdp } 6311+G d \text { log }\end{array}$ & $\begin{array}{l}\text { DMPO-O2 } 180 \text { PCM } \\
\text { o2dp } 1806311+\text { qdpcm.log }\end{array}$ & Pagas & \\
\hline $\begin{array}{r}\text { oxdp_6311+Gd.log } \\
-516.2871198\end{array}$ & $\begin{array}{r}\text { o2dp_180_6311+gdpcm.log } \\
-515.8249272\end{array}$ & $\Delta \Delta$ Gsolv & 354.7261123 \\
\hline $\begin{array}{r}-516.2871198 \\
-516.2622417\end{array}$ & -515.682748 & & -73.60780812 \\
\hline 0.184511 & 0.170028 & & \\
\hline 0.196096 & 0.181448 & & \\
\hline 0.148189 & 0.133638 & & \\
\hline-516.066146 & -515.5013 & & \\
\hline-516.114053 & -515.549109 & & \\
\hline 0.180931 & 0.166729 & & \\
\hline 0.7546 & 0.7566 & & \\
\hline $\mathrm{C} 1$ & C1 & & \\
\hline 0.000000 & 0 & & \\
\hline 2.072340 & 2.20192 & & \\
\hline 1.112730 & 1.03431 & & \\
\hline 0.92072 & 0.92387 & & \\
\hline-516.094603 & -515.646778 & & \\
\hline-516.069725 & -515.504598 & & \\
\hline & -89.21903214 & & \\
\hline-516.142510 & -515.694588 & & \\
\hline-516.117632 & -515.552408 & & \\
\hline
\end{tabular}

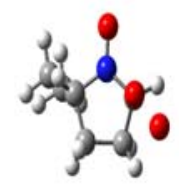

$-515.552408$

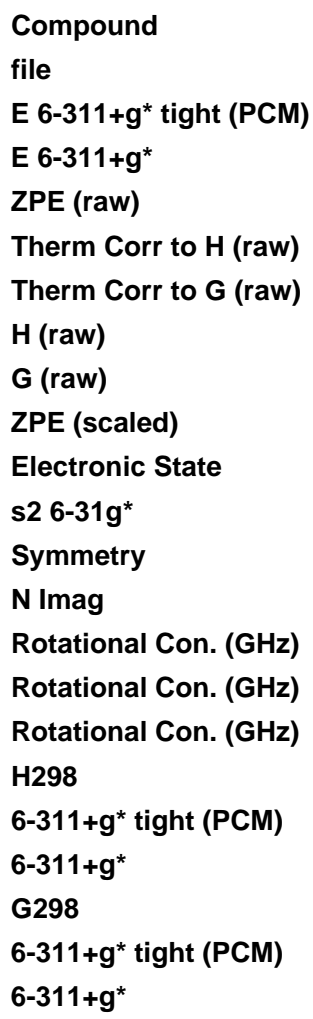



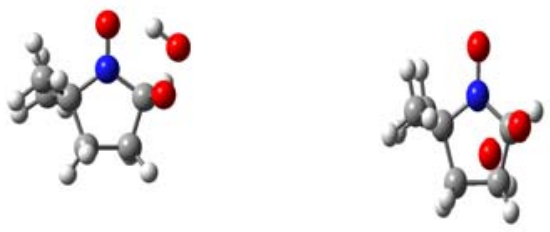

\begin{tabular}{|c|c|c|c|}
\hline Compound & DMPO-OOH adduct & DMPO-O2 292.5 PCM & \\
\hline file & oxdp_6311+Gd.log & o2dp_292_pcm_3.log & 353.6510425 \\
\hline E 6-311+g* tight (PCM) & -516.2871198 & $-515.8225773 \Delta \Delta$ Gsolv & \\
\hline E 6-311+g* & -516.2622417 & -515.6850169 & -70.70923381 \\
\hline ZPE (raw) & 0.184511 & 0.170595 & \\
\hline Therm Corr to $\mathrm{H}$ (raw) & 0.196096 & 0.181826 & \\
\hline Therm Corr to G (raw) & 0.148189 & 0.134849 & \\
\hline H (raw) & -516.066146 & -515.503191 & \\
\hline G (raw) & -516.114053 & -515.550167 & \\
\hline ZPE (scaled) & 0.180931 & 0.167285 & \\
\hline \multicolumn{4}{|l|}{ Electronic State } \\
\hline s2 6-31g* & 0.7546 & 0.7543 & \\
\hline Symmetry & $\mathrm{C} 1$ & C1 & \\
\hline N Imag & 0.000000 & 0 & \\
\hline Rotational Con. (GHz) & 2.072340 & 1.92624 & \\
\hline Rotational Con. (GHz) & 1.112730 & 1.19363 & \\
\hline Rotational Con. (GHz) & 0.92072 & 1.11077 & \\
\hline \multicolumn{4}{|l|}{ H298 } \\
\hline $6-311+g^{*}$ tight $(\mathrm{PCM})$ & -516.094603 & -515.644061 & \\
\hline $6-311+g^{*}$ & -516.069725 & -515.506500 & \\
\hline \multicolumn{4}{|l|}{ G298 } \\
\hline $6-311+g^{*}$ tight $(\mathrm{PCM})$ & -516.142510 & -515.691038 & \\
\hline $6-311+g^{*}$ & -516.117632 & -515.553477 & \\
\hline
\end{tabular}




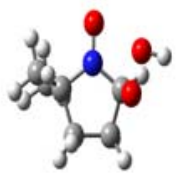

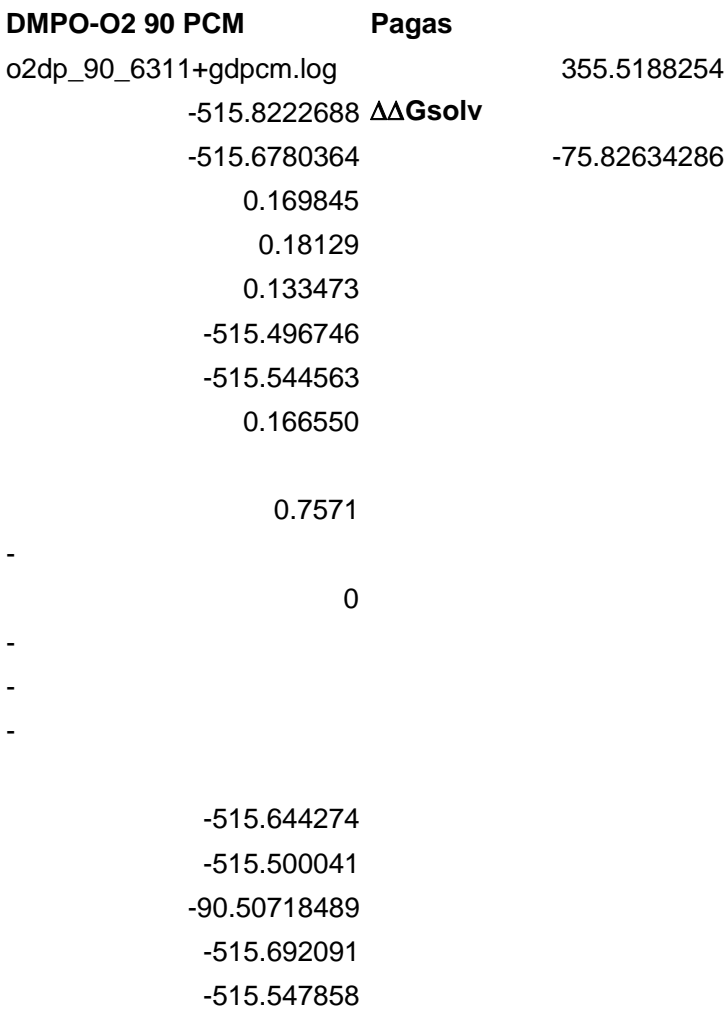

$-515.547858$
66.2 degrees

292b_311+gd.log $-516.2818204$ $-516.258425$

0.183952

0.19588

0.147172

$-516.062545$

$-516.111253$

0.180383

0.7543

0.000000

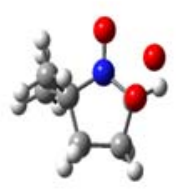

ZPE (scaled)

Electronic State

s2 6-31g*

Symmetry

N Imag

Rotational Con. (GHz)

Rotational Con. (GHz)

Rotational Con. (GHz)

$\mathrm{H} 298$

6-311+g* tight (PCM)

6-311+g*

G298

6-311+g* tight (PCM)

6-311+g*
$-516.089509$

$-516.066114$

$-14.68084203$

$-516.138217$

$-516.114822$ 


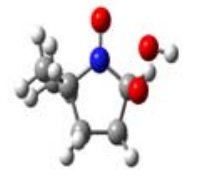

Compound
file
E 6-311+g* tight (PCM)
E 6-311+g*
ZPE (raw)
Therm Corr to H (raw)
Therm Corr to G (raw)
H (raw)
G (raw)
ZPE (scaled)
Electronic State
s2 6-31g*
Symmetry
N Imag
Rotational Con. (GHz)
Rotational Con. (GHz)
Rotational Con. (GHz)
H298
6-311+g* tight (PCM)
6-311+g*
G298
6-311+g* tight (PCM)
6-311+g*

\begin{tabular}{|c|c|c|}
\hline 66.2 degrees & DMPO-02 180 PCM & \\
\hline 292b_311+gd.log & 02dp_180_6311+gdpcm.log & 352.6750833 \\
\hline-516.2818204 & $-515.8249272 \Delta \Delta$ Gsolv & \\
\hline-516.258425 & -515.682748 & -74.5381901 \\
\hline 0.183952 & 0.170028 & \\
\hline 0.19588 & 0.181448 & \\
\hline 0.147172 & 0.133638 & \\
\hline-516.062545 & -515.5013 & \\
\hline-516.111253 & -515.549109 & \\
\hline 0.180383 & 0.166729 & \\
\hline 0.7543 & 0.7566 & \\
\hline - & $\mathrm{C} 1$ & \\
\hline 0.000000 & 0 & \\
\hline - & 2.20192 & \\
\hline - & 1.03431 & \\
\hline - & 0.92387 & \\
\hline-516.089509 & -515.646778 & \\
\hline-516.066114 & -515.504598 & \\
\hline-14.68084203 & -89.21903214 & \\
\hline-516.138217 & -515.694588 & \\
\hline-516.114822 & -515.552408 & \\
\hline
\end{tabular}




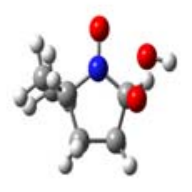

Compound
file
E 6-311+g* tight (PCM)
E 6-311+g*
ZPE (raw)
Therm Corr to H (raw)
Therm Corr to G (raw)
H (raw)
G (raw)
ZPE (scaled)
Electronic State
s2 6-31g*
Symmetry
N Imag
Rotational Con. (GHz)
Rotational Con. (GHz)
Rotational Con. (GHz)
H298
6-311+g* tight (PCM)
6-311+g*
G298
6-311+g* tight (PCM)
6-311+g*

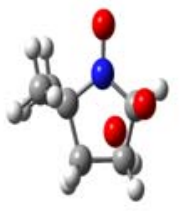

\begin{tabular}{|c|c|c|}
\hline 66.2 degrees & DMPO-O2 292.5 PCM & \\
\hline 292b_311+gd.log & o2dp_292_pcm_3.log & 351.6000135 \\
\hline-516.2818204 & $-515.8225773 \Delta \Delta$ Gsolv & \\
\hline-516.258425 & -515.6850169 & -71.63961579 \\
\hline 0.183952 & 0.170595 & \\
\hline 0.19588 & 0.181826 & \\
\hline 0.147172 & 0.134849 & \\
\hline-516.062545 & -515.503191 & \\
\hline-516.111253 & -515.550167 & \\
\hline 0.180383 & 0.167285 & \\
\hline 0.7543 & 0.7543 & \\
\hline- & C1 & \\
\hline 0.000000 & 0 & \\
\hline- & 1.92624 & \\
\hline- & 1.19363 & \\
\hline- & 1.11077 & \\
\hline-516.089509 & -515.644061 & \\
\hline-516.066114 & -515.506500 & \\
\hline-14.68084203 & & \\
\hline-516.138217 & -515.691038 & \\
\hline-516.114822 & -515.553477 & \\
\hline
\end{tabular}



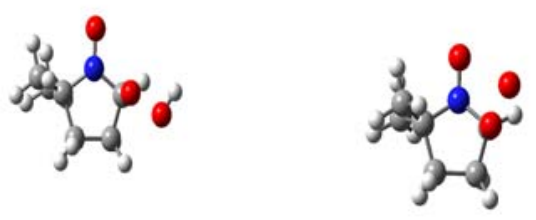

\begin{tabular}{|c|c|c|c|}
\hline Compound & 173 degrees & DMPO-02 90 PCM Pagas & \\
\hline $\begin{array}{l}\text { file } \\
\text { (PCM }\end{array}$ & $\begin{array}{l}\text { 180_311+gd.log } \\
-516.282086\end{array}$ & $\begin{array}{r}\text { 02dp_90_6311+gdpcm.log } \\
-515.8222688 \Delta \Delta \text { Gsolv }\end{array}$ & 356.1739104 \\
\hline E 6-311+g* & -516.259519 & -515.6780364 & -76.34617675 \\
\hline ZPE (raw) & 0.184003 & 0.169845 & \\
\hline Therm Corr to H (raw) & 0.195882 & 0.18129 & \\
\hline Therm Corr to G (raw) & 0.147343 & 0.133473 & \\
\hline H (raw) & -516.063637 & -515.496746 & \\
\hline G (raw) & -516.112176 & -515.544563 & \\
\hline ZPE (scaled) & 0.180433 & 0.166550 & \\
\hline \multicolumn{4}{|l|}{ Electronic State } \\
\hline s2 6-31g* & 0.7542 & 0.7571 & \\
\hline Symmetry & - & - & \\
\hline N Imag & 0.000000 & 0 & \\
\hline Rotational Con. (GHz) & - & & \\
\hline Rotational Con. (GHz) & - & - & \\
\hline Rotational Con. (GHz) & - & - & \\
\hline \multicolumn{4}{|l|}{ H298 } \\
\hline 6-311+g* tight (PCM) & -516.089774 & -515.644274 & \\
\hline $6-311+g^{*}$ & -516.067207 & -515.500041 & \\
\hline G298 & -14.16100814 & -90.50718489 & \\
\hline 6-311+g* tight (PCM) & -516.138313 & -515.692091 & \\
\hline $6-311+g^{*}$ & -516.115746 & -515.547858 & \\
\hline
\end{tabular}



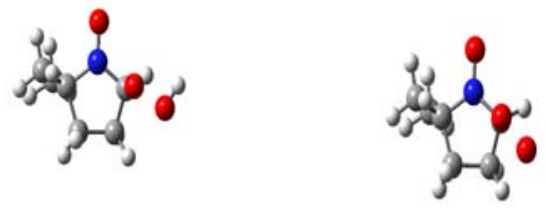

\begin{tabular}{|c|}
\hline Compound \\
\hline file \\
\hline E 6-311+g* tight $(\mathrm{PCM}$ \\
\hline$E$ 6-311+g* \\
\hline ZPE (raw) \\
\hline Therm Corr to $\mathrm{H}$ (raw) \\
\hline Therm Corr to G (raw) \\
\hline H (raw) \\
\hline G (raw) \\
\hline ZPE (scaled) \\
\hline Electronic State \\
\hline s2 6-31g* \\
\hline Symmetry \\
\hline N Imag \\
\hline Rotational Con. (GHz) \\
\hline Rotational Con. (GHz \\
\hline Rotational Con. (G \\
\hline H298 \\
\hline $6-311+g^{*}$ tight $(\mathrm{PCM})$ \\
\hline $6-311+g^{*}$ \\
\hline G298 \\
\hline $6-311+g^{*}$ tight $(\mathrm{PCM}$ \\
\hline $6-311+g^{*}$ \\
\hline
\end{tabular}

173 degrees

180_311+gd.log

$-516.282086$

$-516.259519$

0.184003

0.195882

0.147343

$-516.063637$

$-516.112176$

0.180433

0.7542

0.000000

C1

Pagas

02dp_180_6311+gdpcm.log

$-515.8249272 \Delta \Delta$ Gsolv

$-515.682748$

0.170028

0.181448

0.133638

$-515.5013$

$-515.549109$

0.166729

0.7566

$\begin{array}{rr}0.000000 & 0 \\ 2.20192 \\ 1.03431 \\ 0.92387\end{array}$

$-516.089774$

$-516.067207$

$-515.646778$

$-515.504598$

$-89.21903214$

$-515.694588$

$-515.552408$

$-14.16100814$

$-516.138313$

$-516.115746$

\section{0}

(n)

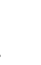

353.3301684

$-75.05802399$ 


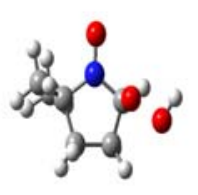

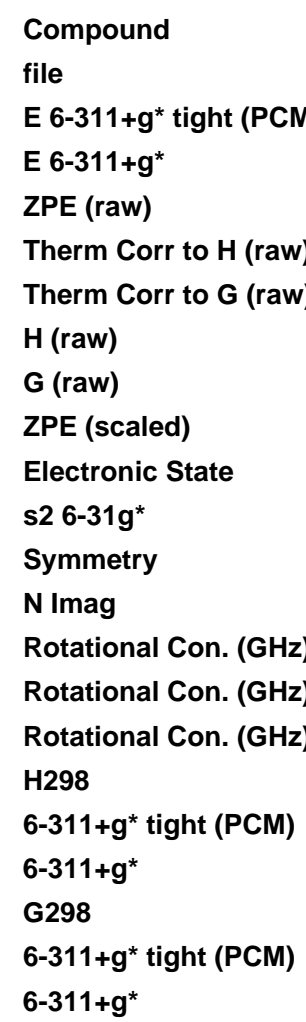

\begin{tabular}{|c|c|c|}
\hline 173 degrees & DMPO-O2 292.5 PCM & \\
\hline 180_311+gd.log & o2dp_292_pcm_3.log & 352.2550985 \\
\hline-516.282086 & $-515.8225773 \Delta \Delta$ Gsolv & \\
\hline-516.259519 & -515.6850169 & -72.15944968 \\
\hline 0.184003 & 0.170595 & \\
\hline 0.195882 & 0.181826 & \\
\hline 0.147343 & 0.134849 & \\
\hline-516.063637 & -515.503191 & \\
\hline-516.112176 & -515.550167 & \\
\hline 0.180433 & 0.167285 & \\
\hline 0.7542 & 0.7543 & \\
\hline 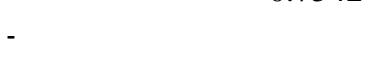 & $\mathrm{C} 1$ & \\
\hline 0.000000 & 0 & \\
\hline - & 1.92624 & \\
\hline- & 1.19363 & \\
\hline- & 1.11077 & \\
\hline-516.089774 & -515.644061 & \\
\hline-516.067207 & -515.506500 & \\
\hline-14.16100814 & & \\
\hline-516.138313 & -515.691038 & \\
\hline-516.115746 & -515.553477 & \\
\hline
\end{tabular}

$-515.553477$

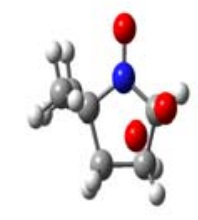




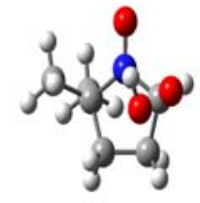

Compound
file
E 6-311+g* tight (PCM)
E 6-311+g*
ZPE (raw)
Therm Corr to H (raw)
Therm Corr to G (raw)
H (raw)
G (raw)
ZPE (scaled)
Electronic State
s2 6-31g*
Symmetry
N Imag
Rotational Con. (GHz)
Rotational Con. (GHz)
Rotational Con. (GHz)
H298
6-311+g* tight (PCM)
6-311+g*
G298
6-311+g* tight (PCM)
6-311+g*

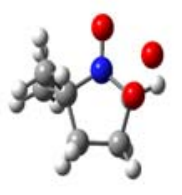

\begin{tabular}{|c|c|c|}
\hline $296.3 \mathrm{deg}$ & DMPO-O2 90 PCM & \\
\hline 67b_311+gd.log & o2dp_90_6311+gdpcm.log & 357.2583679 \\
\hline-516.2871121 & $-515.8222688 \Delta \Delta$ Gsolv & \\
\hline-516.261306 & -515.6780364 & -74.31359567 \\
\hline 0.184063 & 0.169845 & \\
\hline 0.195913 & 0.18129 & \\
\hline 0.147357 & 0.133473 & \\
\hline-516.065393 & -515.496746 & \\
\hline-516.113949 & -515.544563 & \\
\hline 0.180492 & 0.166550 & \\
\hline 0.7543 & 0.7571 & \\
\hline - & - & \\
\hline 0.000000 & 0 & \\
\hline - & - & \\
\hline - & - & \\
\hline - & - & \\
\hline-516.094770 & -515.644274 & \\
\hline-516.068964 & -515.500041 & \\
\hline-16.19358922 & -90.50718489 & \\
\hline-516.143326 & -515.692091 & \\
\hline-516.117520 & -515.547858 & \\
\hline
\end{tabular}




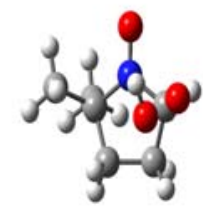

296.3 deg

67b_311+gd.log

file

E 6-311+g* tight (PCM)

E 6-311+g*

ZPE (raw)

Therm Corr to $\mathbf{H}$ (raw)

Therm Corr to G (raw)

H (raw)

G (raw)

ZPE (scaled)

Electronic State

s2 6-31g*

Symmetry

N Imag

Rotational Con. (GHz)

Rotational Con. (GHz)

Rotational Con. (GHz)

H298

6-311+g* tight (PCM)

6-311+g*

G298

6-311+g* tight (PCM)

$6-311+g^{*}$

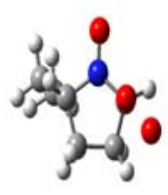

\begin{tabular}{|c|c|c|}
\hline DMPO-02 180 PCM & Pagas & \\
\hline 02dp_180_6311+gdpcm.log & & 354.4146259 \\
\hline-515.8249272 & $\Delta \Delta$ Gsol & \\
\hline-515.682748 & & -73.02544291 \\
\hline 0.170028 & & \\
\hline 0.181448 & & \\
\hline 0.133638 & & \\
\hline-515.5013 & & \\
\hline-515.549109 & & \\
\hline 0.166729 & & \\
\hline 0.7566 & & \\
\hline $\mathrm{C} 1$ & & \\
\hline 0 & & \\
\hline 2.20192 & & \\
\hline 1.03431 & & \\
\hline 0.92387 & & \\
\hline-515.646778 & & \\
\hline-515.504598 & & \\
\hline-89.21903214 & & \\
\hline-515.694588 & & \\
\hline-515.552408 & & \\
\hline
\end{tabular}




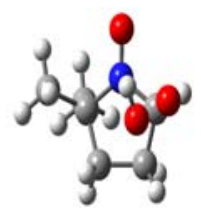

$296.3 \mathrm{deg}$

67b_311+gd.log

$-516.2871121$

$-516.261306$

0.184063

0.195913

0.147357

$-516.065393$

$-516.113949$

0.180492

0.7543

Symmetry

N Imag

Rotational Con. (GHz)

Rotational Con. (GHz)

Rotational Con. (GHz)

H298

6-311+g* tight (PCM)

6-311+g*

G298

6-311 $+g^{*}$ tight (PCM)

$6-311+g^{*}$

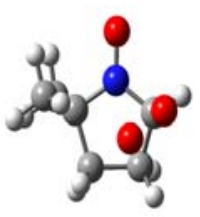

\begin{tabular}{|c|c|c|}
\hline \multicolumn{2}{|l|}{$\begin{array}{l}\text { DMPO-02 292.5 PCM } \\
\text { o2dp_292_pcm_3.log }\end{array}$} & 353.339556 \\
\hline \multicolumn{3}{|c|}{$-515.8225773 \Delta \Delta$ Gsolv } \\
\hline \multirow{2}{*}{\multicolumn{3}{|c|}{0.170595}} \\
\hline & & \\
\hline \multicolumn{3}{|l|}{0.181826} \\
\hline \multicolumn{3}{|l|}{0.134849} \\
\hline \multicolumn{3}{|l|}{-515.503191} \\
\hline \multicolumn{3}{|l|}{-515.550167} \\
\hline \multicolumn{3}{|l|}{0.167285} \\
\hline \multicolumn{3}{|l|}{0.7543} \\
\hline \multicolumn{3}{|l|}{ C1 } \\
\hline 0 & 0 & \\
\hline \multicolumn{3}{|l|}{1.92624} \\
\hline \multicolumn{3}{|l|}{1.19363} \\
\hline \multicolumn{3}{|l|}{1.11077} \\
\hline \multicolumn{3}{|l|}{-515.644061} \\
\hline \multicolumn{3}{|l|}{-515.506500} \\
\hline-515.691038 & & \\
\hline-515.553477 & & \\
\hline
\end{tabular}




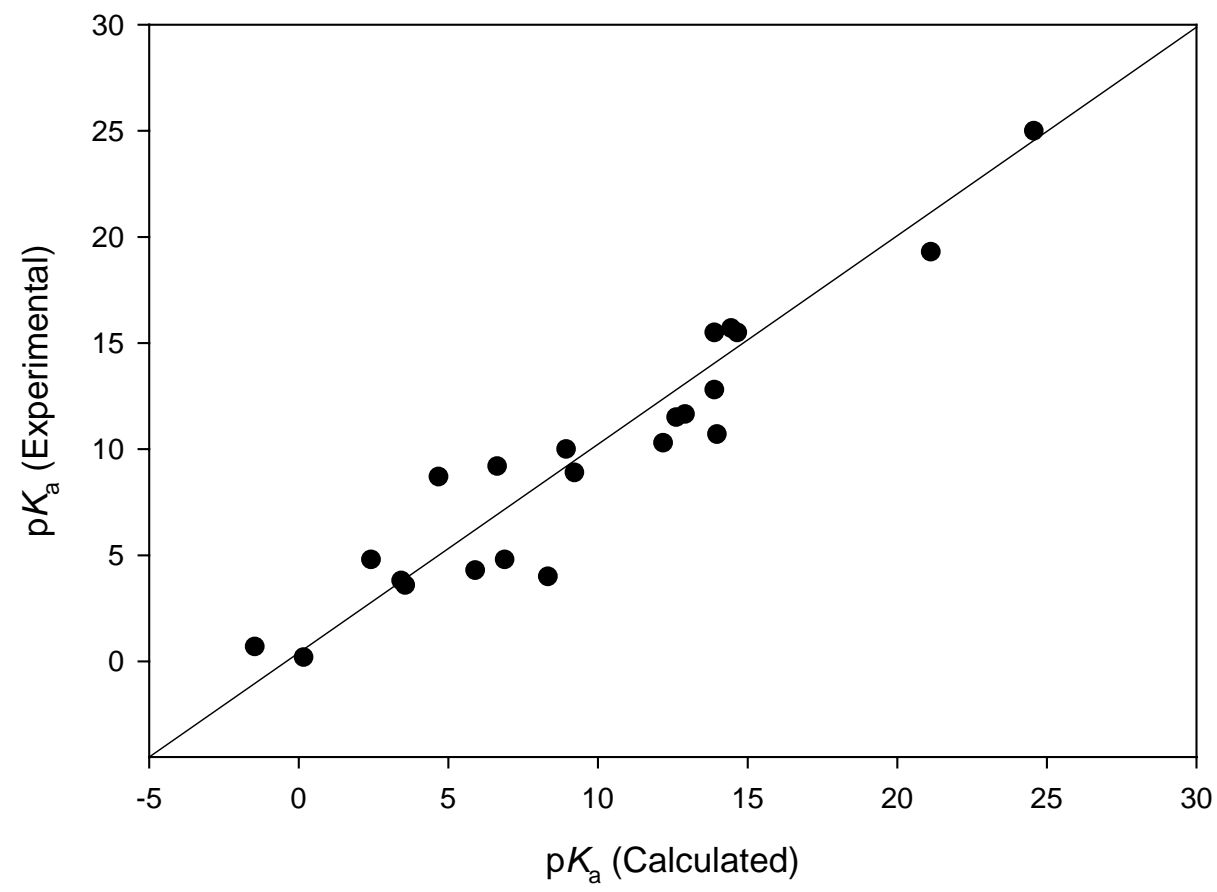

Figure S2. Plot of the experimental $\mathrm{p} K_{\mathrm{a}}$ versus the calculated $\mathrm{p} K_{\mathrm{a}}$. See Table 2 for the thermodynamic parameters. 


\section{Table S7}

Thermodynamic data for the mechanism DMPO-OOH formation at the $B 3 L Y P / 6-311+G(d)$ and PCM/B3LYP/6-311+G(d)//B3LYP/6-311+G(d) level (based on Table 3) 
b3lyp/6-311+g*

ZPE scaling factor $=$

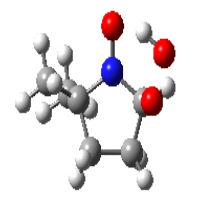

\begin{tabular}{|c|c|c|}
\hline Compound & \multicolumn{2}{|c|}{ DMPO-OOH_A 77.95 ga! DMPO-OOH_A 77.95 PCM } \\
\hline file & 292_311+gd.log & 292_311+gd.log \\
\hline$E$ 6-311+g* & -516.263227 & -516.279588 \\
\hline$E$ 6-311+g* & -516.263227 & -516.263227 \\
\hline ZPE (raw) & 0.18456 & 0.18456 \\
\hline Therm Corr to H (raw) & 0.19615 & 0.19615 \\
\hline Therm Corr to G (raw) & 0.148207 & 0.148207 \\
\hline H (raw) & -516.067077 & -516.067077 \\
\hline G (raw) & -516.11502 & -516.11502 \\
\hline ZPE (scaled) & 0.180980 & 0.180980 \\
\hline \multicolumn{3}{|l|}{ Electronic State } \\
\hline s2 6-31g* & 0.7545 & 0.7545 \\
\hline Symmetry & - & - \\
\hline N Imag & 0.000000 & 0.000000 \\
\hline Rotational Con. (GHz) & - & - \\
\hline Rotational Con. (GHz) & - & - \\
\hline Rotational Con. (GHz) & - & - \\
\hline \multicolumn{3}{|l|}{ H298 } \\
\hline$E$ 6-311+g* & -516.070658 & -516.087018 \\
\hline$E$ 6-311+g* & -516.070658 & -516.070658 \\
\hline G298 & 0 & -10.26665532 \\
\hline$E$ 6-311+g* & -516.118601 & -516.134961 \\
\hline$E$ 6-311+g* & -516.118601 & -516.118601 \\
\hline
\end{tabular}




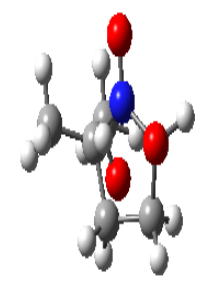

Compound
file
E 6-311+g*
E 6-311+g*
ZPE (raw)
Therm Corr to H (raw)
Therm Corr to G (raw)
H (raw)
G (raw)
ZPE (scaled)
Electronic State
S2 6-31g*
Symmetry
N Imag
Rotational Con. (GHz)
Rotational Con. (GHz)
Rotational Con. (GHz)
H298
E 6-311+g*
E 6-311+g*
G298
E 6-311+g*
E 6-311+g*

DMPO-O2H_B 292.5 gas

67b_311+gd.log

$-516.2613061$

$-516.2613061$

0.184056

0.195917

0.147332

$-516.065389$

$-516.113974$

0.180485

0.7543

0

$-$

-

$-516.068960$

$-516.068960$

$-516.117545$

$-516.117545$

DMPO-O2H_B 292.5 PCM

67b_311+gd.log

$-516.283746$

$-516.261306$

0.184063

0.195913

0.147357

$-516.065393$

$-516.113949$

0.180492

0.7543

0.000000

$-516.091404$

$-516.068964$

$-14.08129937$

$-516.139960$

$-516.117520$ 


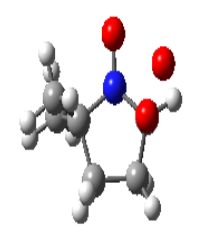

\begin{tabular}{|c|c|c|}
\hline Compound & DMPO-O2 90 gas & $\begin{array}{l}\text { DMPO-O2 } 90 \text { PCM } \\
\text { o2dp } 906311+q d p c m . l o q\end{array}$ \\
\hline file & o2dp_90_6311+gd.log & 02dp_90_6311+gdpcm.log \\
\hline E 6-311+g* & -515.6780364 & -515.782001 \\
\hline E 6-311+g* & -515.6780364 & -515.6780364 \\
\hline ZPE (raw) & 0.169845 & 0.169845 \\
\hline Therm Corr to H (raw) & 0.18129 & 0.18129 \\
\hline Therm Corr to G (raw) & 0.133473 & 0.133473 \\
\hline H (raw) & -515.496746 & -515.496746 \\
\hline G (raw) & -515.544563 & -515.544563 \\
\hline ZPE (scaled) & 0.166550 & 0.166550 \\
\hline \multicolumn{3}{|l|}{ Electronic State } \\
\hline s2 6-31g* & 0.7571 & 0.7571 \\
\hline Symmetry & - & - \\
\hline N Imag & 0 & 0 \\
\hline Rotational Con. (GHz) & - & - \\
\hline Rotational Con. (GHz) & - & - \\
\hline Rotational Con. (GHz) & - & - \\
\hline \multicolumn{3}{|l|}{ H298 } \\
\hline E 6-311+g* & -515.500041 & -515.604006 \\
\hline$E$ 6-311+g* & -515.500041 & -515.500041 \\
\hline G298 & & -65.23875785 \\
\hline$E$ 6-311+g* & -515.547858 & -515.651823 \\
\hline$E$ 6-311+g* & -515.547858 & -515.547858 \\
\hline
\end{tabular}




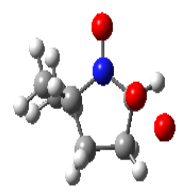

Compound

file

E 6-311+g*

E 6-311+g*

ZPE (raw)

Therm Corr to $\mathbf{H}$ (raw)

Therm Corr to G (raw)

H (raw)

G (raw)

ZPE (scaled)

Electronic State

s2 6-31g*

Symmetry

N Imag

Rotational Con. (GHz)

Rotational Con. (GHz)

Rotational Con. (GHz)

H298

E 6-311+g*

E 6-311+g*

G298

E 6-311+g*

\begin{tabular}{|c|c|c|}
\hline \multicolumn{2}{|c|}{$\begin{array}{l}\text { DMPO-O2 } 180 \text { gas } \\
\text { o2dp_180_6311+gdF.log }\end{array}$} & $\begin{array}{l}\text { DMPO-02 } 180 \text { PCM } \\
\text { o2dp_180_6311+gdpcm.log }\end{array}$ \\
\hline & -515.682748 & -515.785701 \\
\hline & -515.682748 & -515.682748 \\
\hline & 0.170028 & 0.170028 \\
\hline & 0.181448 & 0.181448 \\
\hline & 0.133638 & 0.133638 \\
\hline & -515.5013 & -515.5013 \\
\hline & -515.549109 & -515.549109 \\
\hline & 0.166729 & 0.166729 \\
\hline & 0.7566 & 0.7566 \\
\hline \multirow[t]{10}{*}{ C1 } & & $\mathrm{C} 1$ \\
\hline & 0 & 0 \\
\hline & 2.20192 & 2.20192 \\
\hline & 1.03431 & 1.03431 \\
\hline & 0.92387 & 0.92387 \\
\hline & -515.504598 & -515.607552 \\
\hline & -515.504598 & -515.504598 \\
\hline & & -64.60419451 \\
\hline & -515.552408 & -515.655362 \\
\hline & -515.552408 & -515.552408 \\
\hline
\end{tabular}




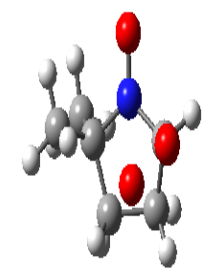

\begin{tabular}{|c|c|c|}
\hline $\begin{array}{l}\text { Compound } \\
\text { file }\end{array}$ & $\begin{array}{l}\text { DMPO-02 292.5 gas } \\
\text { o2dp_292_6311+Gd.log }\end{array}$ & $\begin{array}{l}\text { DMPO-O2 292.5 PCM } \\
\text { o2dp_292_6311+Gdpcm.log }\end{array}$ \\
\hline E 6-311+g* & -515.684966 & -515.780585 \\
\hline E 6-311+g* & -515.684966 & -515.684966 \\
\hline ZPE (raw) & 0.170587 & 0.170587 \\
\hline Therm Corr to $\mathrm{H}$ (raw) & 0.181825 & 0.181825 \\
\hline Therm Corr to G (raw) & 0.134851 & 0.134851 \\
\hline H (raw) & -515.503141 & -515.503141 \\
\hline G (raw) & -515.550115 & -515.550115 \\
\hline ZPE (scaled) & 0.167278 & 0.167278 \\
\hline \multicolumn{3}{|l|}{ Electronic State } \\
\hline s2 6-31g* & 0.7546 & 0.7546 \\
\hline Symmetry & C1 & $\mathrm{C} 1$ \\
\hline N Imag & 0 & \\
\hline Rotational Con. (GHz) & 1.92624 & 1.92624 \\
\hline Rotational Con. (GHz) & 1.19363 & 1.19363 \\
\hline Rotational Con. (GHz) & 1.11077 & 1.11077 \\
\hline \multicolumn{3}{|l|}{ H298 } \\
\hline E 6-311+g* & -515.506450 & -515.602069 \\
\hline$E$ 6-311+g* & -515.506450 & -515.506450 \\
\hline G298 & & -60.0018472 \\
\hline E 6-311+g* & -515.553424 & -515.649043 \\
\hline E 6-311+g* & -515.553424 & -515.553424 \\
\hline
\end{tabular}




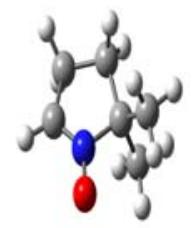

\begin{tabular}{|c|c|c|}
\hline $\begin{array}{l}\text { Compound } \\
\text { file }\end{array}$ & $\begin{array}{l}\text { DMPO gas } \\
\text { dmpo_6_311+Gd.log }\end{array}$ & DMPO PCM \\
\hline E 6-311+g* & -365.2687238 & -365.279311 \\
\hline E 6-311+g* & -365.2687238 & -365.2687238 \\
\hline ZPE (raw) & 0.165591 & 0.165591 \\
\hline Therm Corr to $\mathrm{H}$ (raw) & 0.174526 & 0.174526 \\
\hline Therm Corr to G (raw) & 0.133777 & 0.133777 \\
\hline H (raw) & -365.094198 & -365.094198 \\
\hline G (raw) & -365.134947 & -365.134947 \\
\hline ZPE (scaled) & 0.162379 & 0.162379 \\
\hline \multicolumn{3}{|l|}{ Electronic State } \\
\hline s2 6-31g* & 0.000000 & 0.000000 \\
\hline Symmetry & - & - \\
\hline N Imag & 0.000000 & 0.000000 \\
\hline Rotational Con. (GHz) & - & - \\
\hline Rotational Con. (GHz) & - & - \\
\hline Rotational Con. (GHz) & - & - \\
\hline \multicolumn{3}{|l|}{ H298 } \\
\hline$E$ 6-311+g* & -365.097410 & -365.107997 \\
\hline E 6-311+g* & -365.097410 & -365.097410 \\
\hline \multicolumn{3}{|l|}{ G298 } \\
\hline E 6-311+g* & -365.138159 & -365.148746 \\
\hline$E$ 6-311+g* & -365.138159 & -365.138159 \\
\hline
\end{tabular}




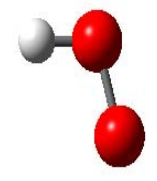

\begin{tabular}{|c|c|c|}
\hline $\begin{array}{l}\text { Compound } \\
\text { file }\end{array}$ & $\begin{array}{l}\text { OOH radical gas } \\
\text { peroxyl_6311+Gd.log }\end{array}$ & $\mathrm{OOH}$ radical PCM \\
\hline$E$ 6-311+g* & -150.951952 & -150.966826 \\
\hline$E$ 6-311+g* & -150.951952 & -150.951952 \\
\hline ZPE (raw) & 0.014108 & 0.014108 \\
\hline Therm Corr to $\mathrm{H}$ (raw) & 0.017910 & 0.017910 \\
\hline Therm Corr to G (raw) & -0.008064 & -0.008064 \\
\hline H (raw) & -150.934042 & -150.934042 \\
\hline G (raw) & -150.960016 & -150.960016 \\
\hline ZPE (scaled) & 0.013834 & 0.013834 \\
\hline \multicolumn{3}{|l|}{ Electronic State } \\
\hline s2 6-31g* & 0.753800 & 0.753800 \\
\hline Symmetry & - & - \\
\hline N Imag & 0.000000 & 0.000000 \\
\hline Rotational Con. (GHz) & - & - \\
\hline Rotational Con. (GHz) & - & - \\
\hline Rotational Con. (GHz) & - & - \\
\hline \multicolumn{3}{|l|}{ H298 } \\
\hline$E$ 6-311+g* & -150.934316 & -150.949190 \\
\hline$E$ 6-311+g* & -150.934316 & -150.934316 \\
\hline \multicolumn{3}{|l|}{ G298 } \\
\hline$E$ 6-311+g* & -150.960290 & -150.975164 \\
\hline$E$ 6-311+g* & -150.960290 & -150.960290 \\
\hline
\end{tabular}




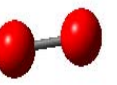

\begin{tabular}{|c|c|c|}
\hline $\begin{array}{l}\text { Compound } \\
\text { file }\end{array}$ & $\begin{array}{l}\text { O2 rad anion gas } \\
02 \_6311+G d . l o g\end{array}$ & 02 rad anion PCM \\
\hline E 6-311+g* & -150.3917078 & -150.516179 \\
\hline$E$ 6-311+g* & -150.3917078 & -150.3917078 \\
\hline ZPE (raw) & 0.002655 & 0.002655 \\
\hline Therm Corr to $\mathrm{H}$ (raw) & 0.005979 & 0.005979 \\
\hline Therm Corr to G (raw) & -0.017138 & -0.017138 \\
\hline H (raw) & -150.385729 & -150.385729 \\
\hline G (raw) & -150.408846 & -150.408846 \\
\hline ZPE (scaled) & 0.002603 & 0.002603 \\
\hline \multicolumn{3}{|l|}{ Electronic State } \\
\hline s2 6-31g* & 0.756500 & 0.756500 \\
\hline Symmetry & - & \\
\hline N Imag & 0 & 0 \\
\hline Rotational Con. (GHz) & - & - \\
\hline Rotational Con. (GHz) & - & - \\
\hline Rotational Con. (GHz) & - & - \\
\hline \multicolumn{3}{|l|}{ H298 } \\
\hline$E$ 6-311+g* & -150.385780 & -150.510252 \\
\hline E 6-311+g* & -150.385780 & -150.385780 \\
\hline \multicolumn{3}{|l|}{ G298 } \\
\hline E 6-311+g* & -150.408897 & -150.533369 \\
\hline$E$ 6-311+g* & -150.408897 & -150.408897 \\
\hline
\end{tabular}




\begin{tabular}{|c|c|c|}
\hline $\begin{array}{l}\text { Compound } \\
\text { file }\end{array}$ & $\begin{array}{l}\text { Hydroxide ion gas } \\
\text { hydroxide_6311+Gd.log }\end{array}$ & $\begin{array}{l}\text { Hydroxide ion PCM } \\
\text { hydroxide_6311+Gdpcm.log }\end{array}$ \\
\hline$E$ 6-311+g* & -75.81812211 & -75.953036 \\
\hline$E$ 6-311+g* & -75.81812211 & -75.81812211 \\
\hline ZPE (raw) & 0.01 & 0.01 \\
\hline Therm Corr to H (raw) & 0.011580 & 0.011580 \\
\hline Therm Corr to G (raw) & -0.007992 & -0.007992 \\
\hline H (raw) & -75.806542 & -75.806542 \\
\hline G (raw) & -75.826114 & -75.826114 \\
\hline ZPE (scaled) & 0.008115 & 0.008115 \\
\hline \multicolumn{3}{|l|}{ Electronic State } \\
\hline s2 6-31g* & 0 & \\
\hline Symmetry & - & - \\
\hline N Imag & 0 & \\
\hline \multicolumn{3}{|l|}{ Rotational Con. (GHz) } \\
\hline \multicolumn{3}{|l|}{ Rotational Con. (GHz) } \\
\hline \multicolumn{3}{|l|}{ Rotational Con. (GHz) } \\
\hline \multicolumn{3}{|l|}{ H298 } \\
\hline$E$ 6-311+g* & -75.806703 & -75.941617 \\
\hline$E$ 6-311+g* & -75.806703 & -75.806703 \\
\hline \multicolumn{3}{|l|}{ G298 } \\
\hline$E$ 6-311+g* & -75.826275 & -75.961189 \\
\hline$E$ 6-311+g* & -75.826275 & -75.826275 \\
\hline
\end{tabular}




\section{as}

\begin{tabular}{|c|c|c|}
\hline Compound & Water gas & Water PCM \\
\hline file & H2O_6311+Gd.log & H2O_6311+Gdpcm.log \\
\hline E 6-311+g* & -76.44380909 & -76.456308 \\
\hline E 6-311+g* & -76.44380909 & -76.44380909 \\
\hline ZPE (raw) & 0.02 & 0.02 \\
\hline Therm Corr to $\mathrm{H}$ (raw) & 0.024994 & 0.024994 \\
\hline Therm Corr to G (raw) & 0.002921 & 0.002921 \\
\hline H (raw) & -76.418815 & -76.418815 \\
\hline G (raw) & -76.440888 & -76.440888 \\
\hline ZPE (scaled) & 0.020803 & 0.020803 \\
\hline \multicolumn{3}{|l|}{ Electronic State } \\
\hline s2 6-31g* & 0 & 0 \\
\hline \multicolumn{3}{|l|}{ Symmetry } \\
\hline N Imag & 0 & 0 \\
\hline \multicolumn{3}{|l|}{ Rotational Con. (GHz) } \\
\hline \multicolumn{3}{|l|}{ Rotational Con. (GHz) } \\
\hline \multicolumn{3}{|l|}{ Rotational Con. (GHz) } \\
\hline \multicolumn{3}{|l|}{ H298 } \\
\hline$E$ 6-311+g* & -76.419227 & -76.431726 \\
\hline E 6-311+g* & -76.419227 & -76.419227 \\
\hline \multicolumn{3}{|l|}{ G298 } \\
\hline E 6-311+g* & -76.441300 & -76.453799 \\
\hline E 6-311+g* & -76.441300 & -76.441300 \\
\hline
\end{tabular}




\section{Table S8 \\ Transition state data for the DMPO-OOH \\ formation at the B3LYP/6-31+G(d,p)//B3LYP/6-31G(d) level in gas and aqueous phases \\ (based on Table 4)}




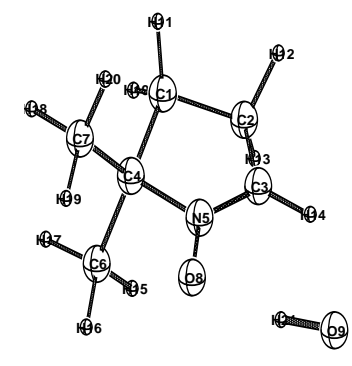

Compound

file

E 6-31+g** tight

E 6-31g*

ZPE (raw)

Therm Corr to $\mathbf{H}$ (raw)

Therm Corr to G (raw)

H (raw)

G (raw)

ZPE (scaled)

Electronic State

s2 6-31g*

Symmetry

N Imag

Rotational Con. (GHz)

Rotational Con. (GHz)

Rotational Con. (GHz)

H298

E 6-31+g ${ }^{\star *}$ tight

E 6-31g*

G298

E 6-31+g ${ }^{\star *}$ tight

E 6-31g*
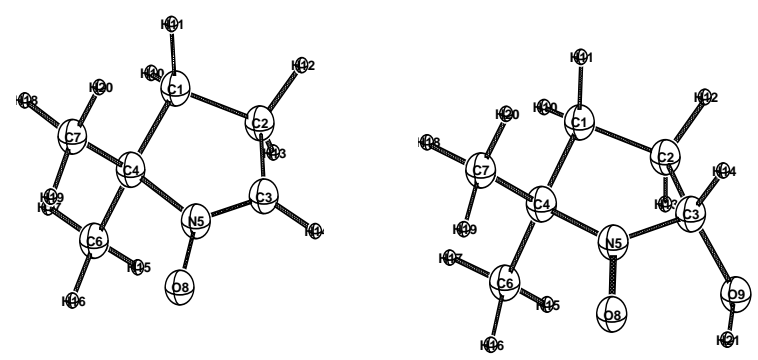

102

DMPO-OH TS

dmpohCO1_8A_F.log
DMPO-OH C-O pos dmpohCOpos.log
$-440.9600703$

$-440.9168394$

0.178115

0.188746

0.142863

$-440.728093$

$-440.773976$

0.174660

0.7521

C1

0

$-440.776138$

$-440.734673$

0.00

$-440.823757$

$-440.782292$

0.7541

1

$-440.774780$

$-440.731549$

1.94

$-440.820663$

$-440.777432$
$-441.0345875$

$-440.993606$

0.182147

0.192612

0.147306

$-440.800994$

$-440.8463$

0.178613

0.7536

0

-

$-$

$-440.845509$
$-440.804528$

$-42.08$

$-440.890815$

$-440.849834$ 

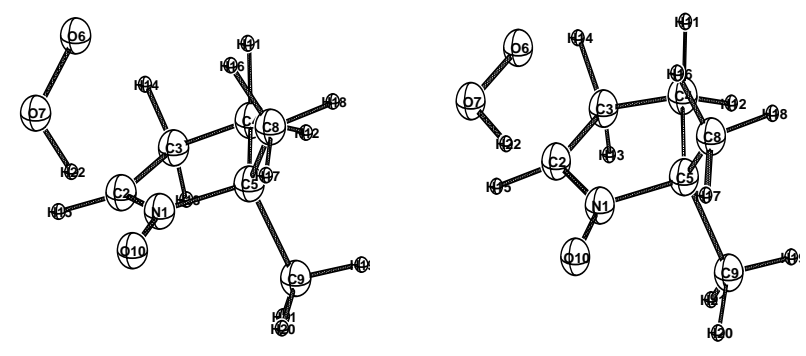

ZPE (scaled)

Electronic State

s2 6-31g*

Symmetry

N Imag

Rotational Con. (GHz)

Rotational Con. (GHz)

Rotational Con. (GHz)

H298

E 6-31+g** tight

E 6-31g*

G298

E 6-31+g** tight

E 6-31g*
DMPO-OOH C-O neg

oxdmpoOOTS-freq.log

$$
\begin{array}{r}
-516.145354 \\
-516.1014391 \\
0.183387 \\
0.195917 \\
0.14445 \\
-515.905522 \\
-515.956989 \\
0.179829
\end{array}
$$

0.753

C1

C1

0
DMPO-OOH TS

oxdmpoOOTS.log

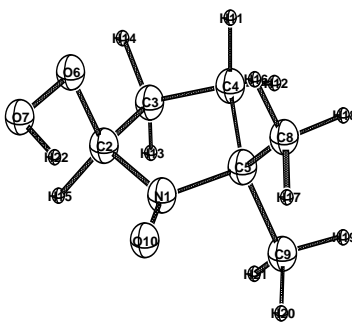

DMPO-OOH C-O pos

oxdmpoOOTS+freq.log

$-516.1668362$

$-516.1253238$

0.18595

0.197339

0.149905

$-515.927985$

$-515.975419$

0.182343

0.7538

C1

1

0

$-515.936753$

12.40

$-515.973105$

$-515.931592$

$-10.09$

$-515.984707$

$-515.941342$

$-516.020539$

$-515.979026$
$-515.893388$ 

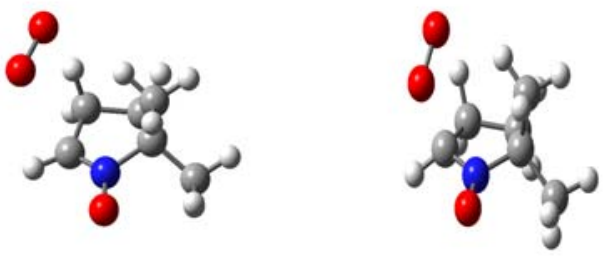

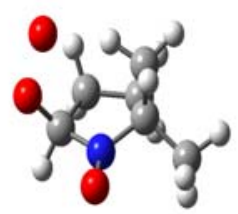

\author{
Compound \\ file \\ E 6-31+g ${ }^{\star *}$ tight \\ E 6-31g* \\ ZPE (raw) \\ Therm Corr to $\mathrm{H}$ (raw) \\ Therm Corr to G (raw) \\ H (raw) \\ G (raw) \\ ZPE (scaled) \\ Electronic State \\ s2 6-31g* \\ Symmetry \\ N Imag \\ Rotational Con. (GHz) \\ Rotational Con. (GHz) \\ Rotational Con. (GHz) \\ H298 \\ E 6-31+g ${ }^{\star *}$ tight \\ E 6-31g* \\ G298 \\ E 6-31+g** tight \\ E 6-31g*
}

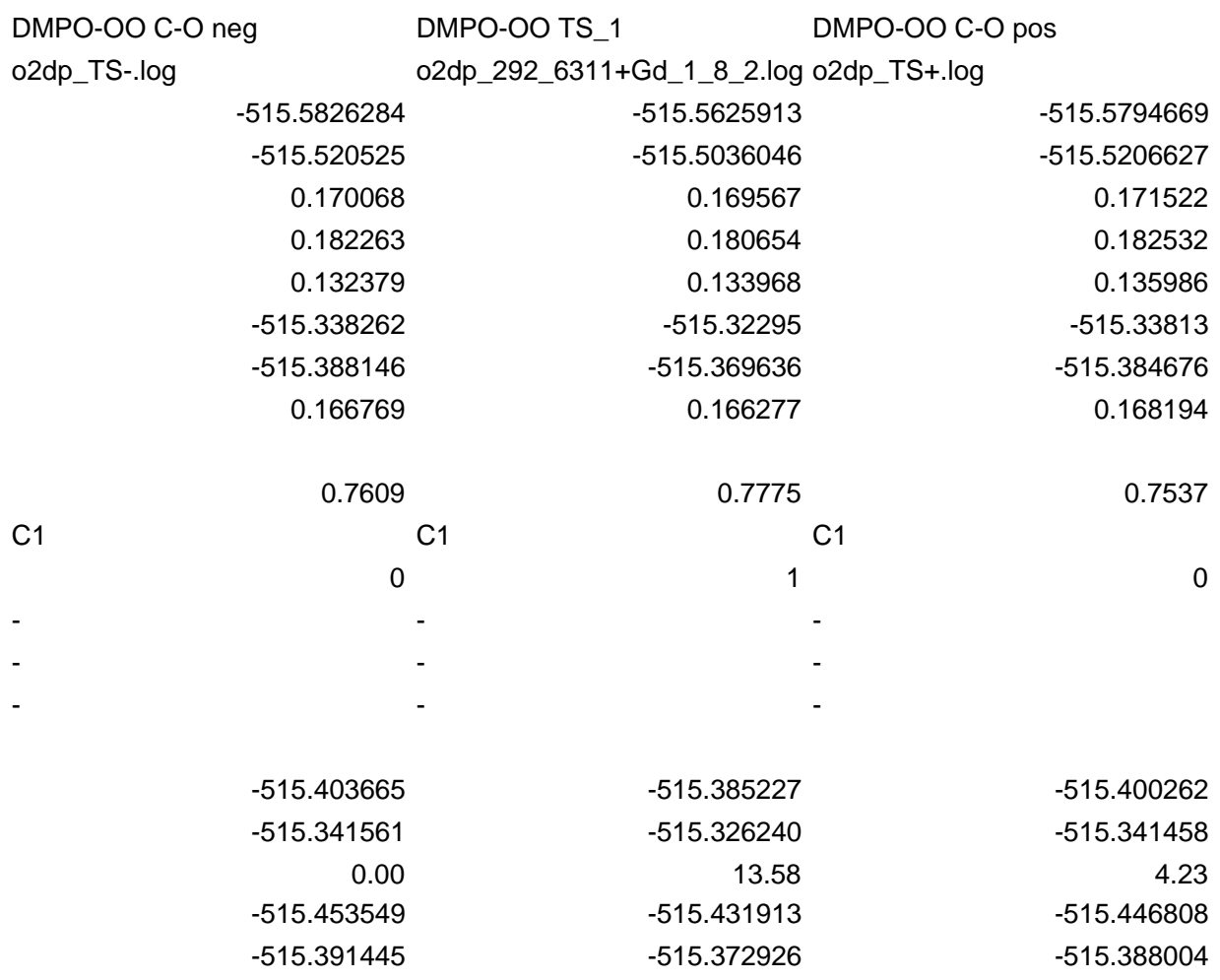




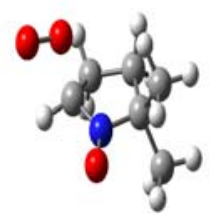

Compound
file
E 6-31+g** tight
E 6-31g*
ZPE (raw)
Therm Corr to H (raw)
Therm Corr to G (raw)
H (raw)
G (raw)
ZPE (scaled)
Electronic State
s2 6-31g*
Symmetry
N Imag
Rotational Con. (GHz)
Rotational Con. (GHz)
Rotational Con. (GHz)
H298
E 6-31+g** tight
E 6-31g*
G298
E 6-31+g** tight
E 6-31g*

$\begin{array}{cr}\begin{array}{c}\text { DMPO-OO C-O pos } \\ \text { o2dpts_TS2+.log }\end{array} & \text { DMPO-OO TS_ } \\ -515.5795221 \\ -515.5147136 \\ 0.170026 \\ 0.182364 \\ 0.131444 \\ -515.33235 \\ -515.383269 \\ \\ 0.7596\end{array}$

C1

C1

0

-515.567184
-515.502376
0.00
-515.618104
-515.553296
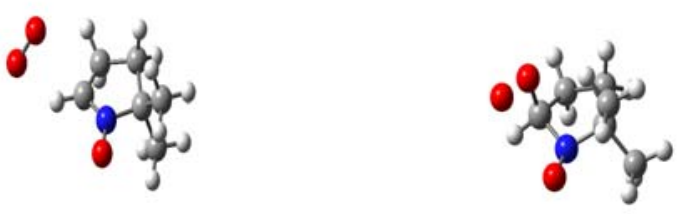

DMPO-OO C-O neg

o2dpts_TS2-.log

$-515.5624614$

$-515.5009754$

0.169144

0.18056

0.132467

$-515.320415$

$-515.368508$

0.758

0.7558

$-515.57378$

$-515.5118169$

0.171167

0.182432

0.135168

$-515.329385$

$-515.376649$

C1

1

0

$-515.551045$

$-515.562515$

$-515.489559$

$-515.500552$

11.90

5.22

$-515.599138$

$-515.609779$

$-515.537652$

$-515.547816$ 

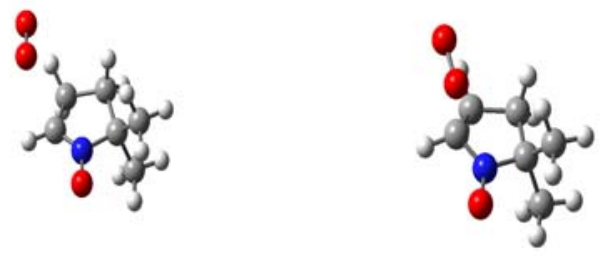

DMPO-OO TS_3
o2dpts_TS3.log
-515.581414
-515.5180525
0.16992
0.182277
0.131066
-515.335776
-515.386987
0.7626

DMPO-OO TS_3

DMPO-OO C-O neg

o2dpts_TS3-.log

file

E 6-31+g ${ }^{\star *}$ tight

E 6-31g*

ZPE (raw)

Therm Corr to $\mathbf{H}$ (raw)

Therm Corr to G (raw)

H (raw)

G (raw)

ZPE (scaled)

Electronic State

s2 6-31g*

Symmetry

N Imag

Rotational Con. (GHz)

Rotational Con. (GHz)

Rotational Con. (GHz)

H298

E 6-31+g** tight

E 6-31g*

G298

E 6-31+g** tight

E 6-31g*
C1

$-515.5653048$

$-515.5038473$

0.180651

0.132648

$-515.323197$

$-515.3712$

0.7657

C1

1

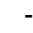

$-515.569057$

$-515.505695$

0.00

$-515.620268$

$-515.556906$
0.169309
DMPO-OO C-O pos

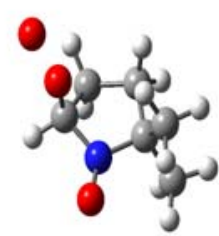

02dpts_TS3+.log

$-515.5792885$

$-515.5155175$

0.171201

0.182435

0.13532

$-515.333082$

$-515.380198$

0.7574

$-515.568054$

$-515.504284$

3.20

$-515.615169$

$-515.551399$ 

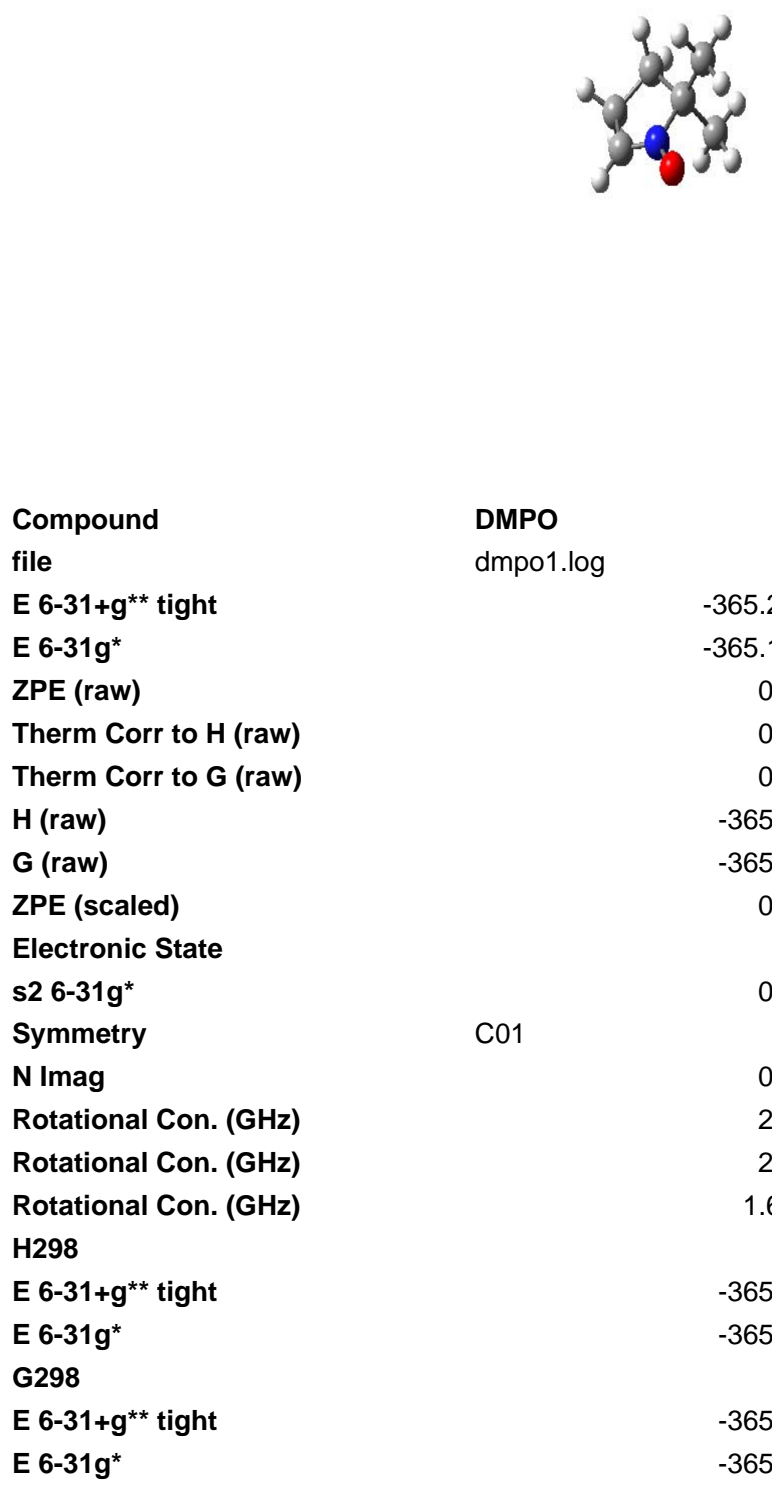

\begin{tabular}{lr} 
DMPO & \multicolumn{1}{c}{ O2 Radical } \\
dmpo1.log
\end{tabular}

\section{$\infty$}

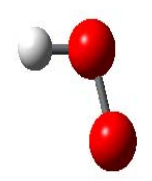

\begin{tabular}{rr}
\multicolumn{2}{c}{ OoH Radical } \\
peroxylrad.log \\
-150.3493642 & -150.9154259 \\
-150.2996938 & -150.899156 \\
0.002761 & 0.014031 \\
0.006082 & 0.017832 \\
-0.017041 & -0.008160 \\
-150.293612 & -150.881325 \\
-150.316735 & -150.907317 \\
0.002707 & 0.013759 \\
& \\
0.7528 & 0.752700 \\
C01 & \\
0 & 0.000000 \\
1.63425 & \\
0.75785 & \\
0.66048 & \\
-150.343336 & -150.897866 \\
-150.293665 & -150.881597 \\
-150.366459 & -150.923858 \\
-150.316788 & -150.907589
\end{tabular}




Compound
file
E 6-31+g** tight
E 6-31g*
ZPE (raw)
Therm Corr to H (raw)
Therm Corr to G (raw)
H (raw)
G (raw)
ZPE (scaled)
Electronic State
S2 6-31g*
Symmetry
N Imag
Rotational Con. (GHz)
Rotational Con. (GHz)
Rotational Con. (GHz)
H298
E 6-31+g** tight
E 6-31g*
G298
E 6-31+g** tight
E 6-31g*

OH

OHfreq.LOG

$-75.73900594$

$-75.72215961$

0.008304

0.011609

$-0.008638$

$-75.711846$

$-75.732093$

0.008143

0.751967

$C * V$

0.000000

$-75.727558$

$-75.710712$

$-75.747805$

$-75.730959$ 


DMPO + O2
DMPO + OOH
DMPO + OH
DMPO----O2 complex
DMPO----OOH complex
DMPO----OH complex
DMPO-O2 TS
DMPO-O2H TS
DMPO-OH TS
DMPO-O2
DMPO-OOH
DMPO-OH
DMPO + O2
DMPO + OOH
DMPO + OH
DMPO----O2 complex
DMPO----OOH complex
DMPO----OH complex
DMPO-O2 TS
DMPO-OH TS
DMPO-OH
DMPO-OOH
DMPO
DMPO
DMPO

$\mathrm{DH}$ DG

$\begin{array}{rr}-515.5572811 & -515.442716 \\ -516.123343 & -516.000115 \\ -440.9469228 & -440.824062 \\ & \\ -515.5826284 & -515.4535487 \\ -516.145354 & -516.0044618 \\ -440.9624931 & -440.8237568 \\ & \\ -515.5625913 & -515.4319129 \\ -516.1280954 & -515.984707 \\ -440.9600703 & -440.8206627 \\ -515.5794669 & -515.4468085 \\ -516.1668362 & -516.0205387 \\ -441.0345875 & -440.8908151\end{array}$

DH

\begin{tabular}{rr}
\multicolumn{1}{c}{ DG } & \\
0.0 & 0.0 \\
0.0 & 0.0 \\
0.0 & 0.0 \\
& \\
-15.9 & -6.8 \\
-13.8 & -2.7 \\
-9.8 & 0.2 \\
& \\
-3.3 & 6.8 \\
-3.0 & 9.7 \\
-8.3 & 2.1 \\
& \\
-13.9 & -2.6 \\
-27.3 & -12.8 \\
-55.0 & -41.9
\end{tabular}



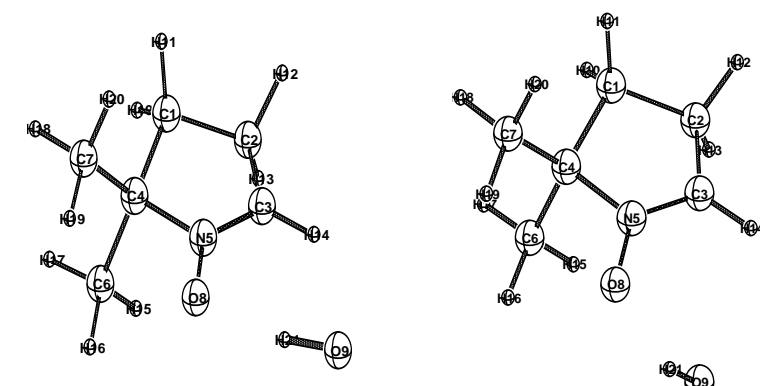

10.

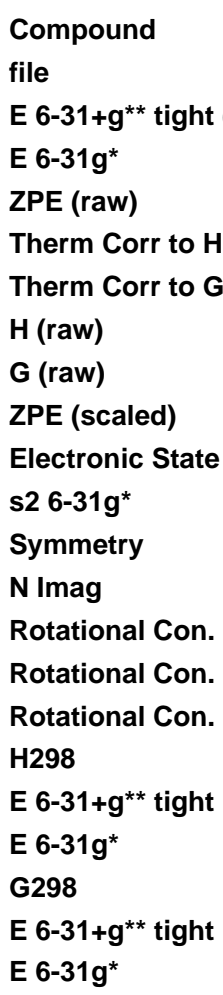

\begin{tabular}{|c|c|c|}
\hline $\begin{array}{l}\text { DMPO-OH C-O neg } \\
\text { dmpohCOneg.log }\end{array}$ & $\begin{array}{l}\text { DMPO-OH TS } \\
\text { dmpohCO1_8A_F.log }\end{array}$ & $\begin{array}{l}\text { DMPO-OH C-O pos } \\
\text { dmpohCOpos.log }\end{array}$ \\
\hline-440.95854 & -440.959491 & -441.035643 \\
\hline-440.9210284 & -440.9168394 & -440.993606 \\
\hline 0.178537 & 0.178115 & 0.182147 \\
\hline 0.189819 & 0.188746 & 0.192612 \\
\hline 0.1422 & 0.142863 & 0.147306 \\
\hline-440.73121 & -440.728093 & -440.800994 \\
\hline-440.778828 & -440.773976 & -440.8463 \\
\hline 0.175073 & 0.174660 & 0.178613 \\
\hline 0.7521 & 0.7541 & 0.7536 \\
\hline- & $\mathrm{C} 1$ & - \\
\hline 0 & 1 & 0 \\
\hline- & - & - \\
\hline - & - & - \\
\hline - & - & - \\
\hline-440.772185 & -440.774200 & -440.846565 \\
\hline-440.734673 & -440.731549 & -440.804528 \\
\hline 0.00 & -0.18 & -45.22 \\
\hline-440.819804 & -440.820083 & -440.891871 \\
\hline-440.782292 & -440.777432 & -440.849834 \\
\hline
\end{tabular}

$-440.891871$

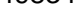

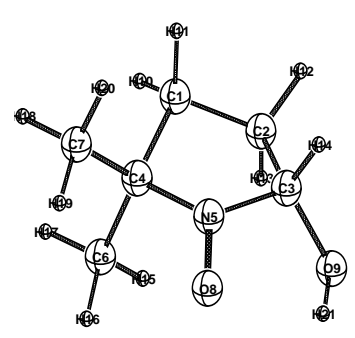

0 


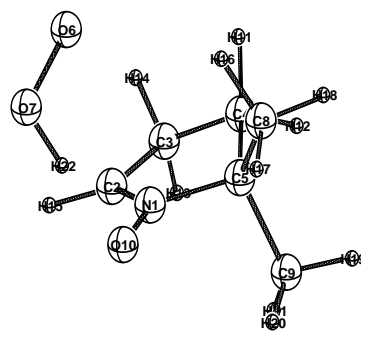

Compound

file

E 6-31 $+g^{* *}$ tight (PCM)

E 6-31g*

ZPE (raw)

Therm Corr to $\mathbf{H}$ (raw)

Therm Corr to G (raw)

H (raw)

G (raw)

ZPE (scaled)

Electronic State

s2 6-31g*

Symmetry

N Imag

Rotational Con. (GHz)

Rotational Con. (GHz)

Rotational Con. (GHz)

H298

E 6-31+g ${ }^{* *}$ tight

E 6-31g*

G298

E 6-31+g** tight

E 6-31g*

DMPO-OOH C-O neg

oxdmpoOOTS-freq.log

C1
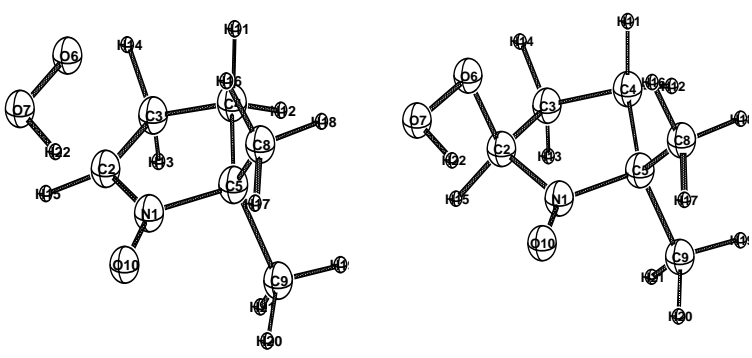

$-516.143242$

$-516.1014391$

0.183387

0.195917

0.14445

$-515.905522$

$-515.956989$

0.179829

0.753

C1

DMPO-OOH TS

oxdmpoOOTS.log

$-516.128933$

$-516.0847309$

0.183278

0.194898

0.146944

$-515.889832$

$-515.937787$

0.179722

DMPO-OOH C-O pos

oxdmpoOOTS+freq.log

$-516.165541$

$-516.1253238$

0.18595

0.197339

0.149905

$-515.927985$

$-515.975419$

0.182343

0.7942

C1

0.7538

1

0

$-515.950883$

$-515.909080$

0.00

$-515.937591$

$-515.971809$

$-515.931592$

$-10.60$

$-516.002350$

$-515.960547$
$-515.893388$

10.55

$-515.985545$

$-515.941342$
$-516.019243$

$-515.979026$ 

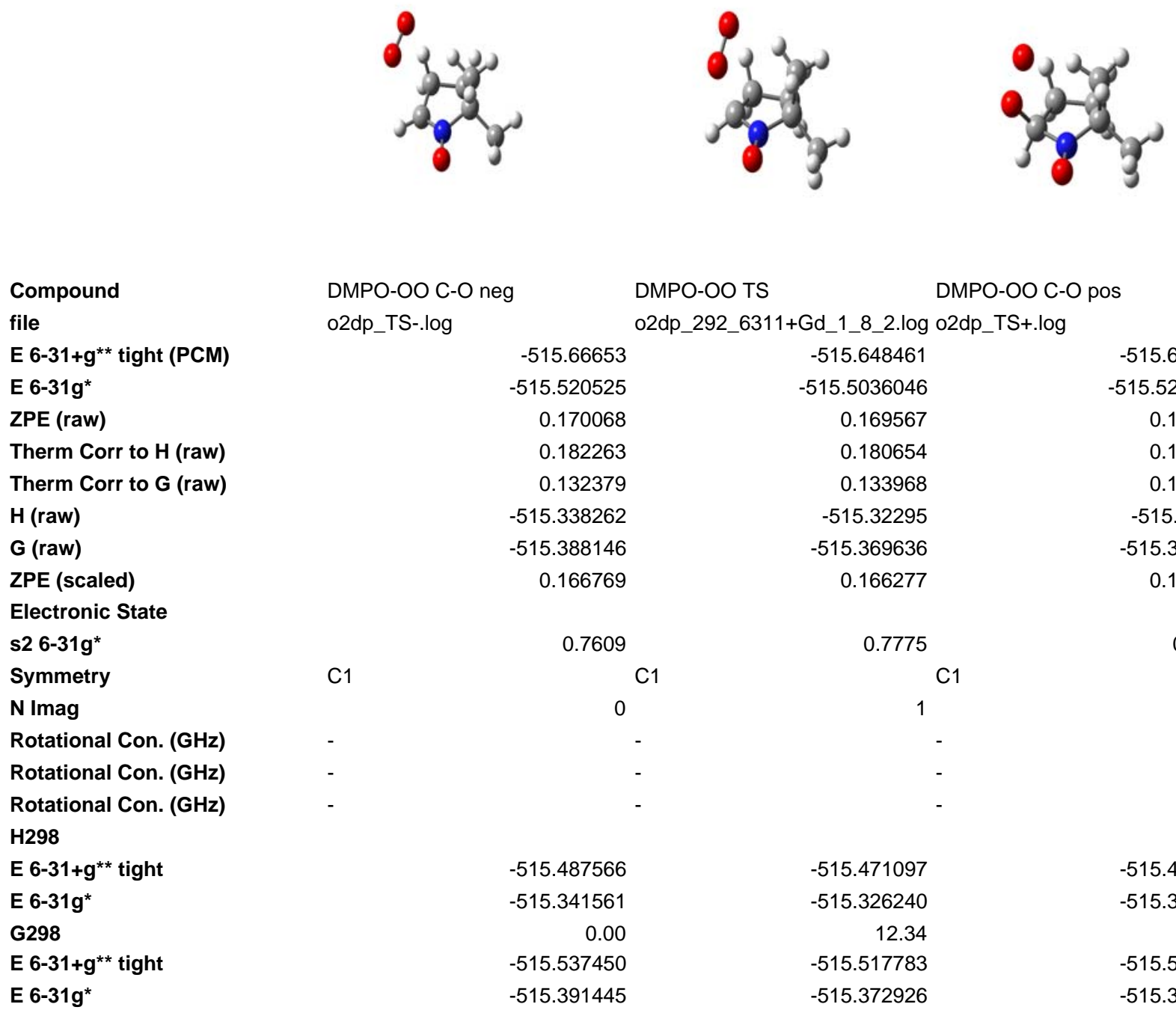

$-515.388004$ 


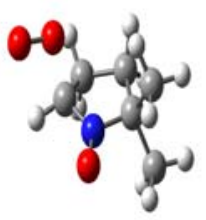

Compound
file
E 6-31+g** tight (PCM)
E 6-31g*
ZPE (raw)
Therm Corr to H (raw)
Therm Corr to G (raw)
H (raw)
G (raw)
ZPE (scaled)
Electronic State
s2 6-31g*
Symmetry
N Imag
Rotational Con. (GHz)
Rotational Con. (GHz)
Rotational Con. (GHz)
H298
E 6-31+g** tight
E 6-31g*
G298
E 6-31+g* tight
E 6-31g*
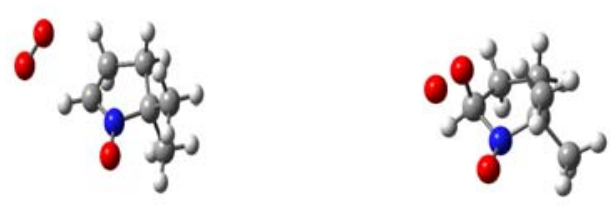

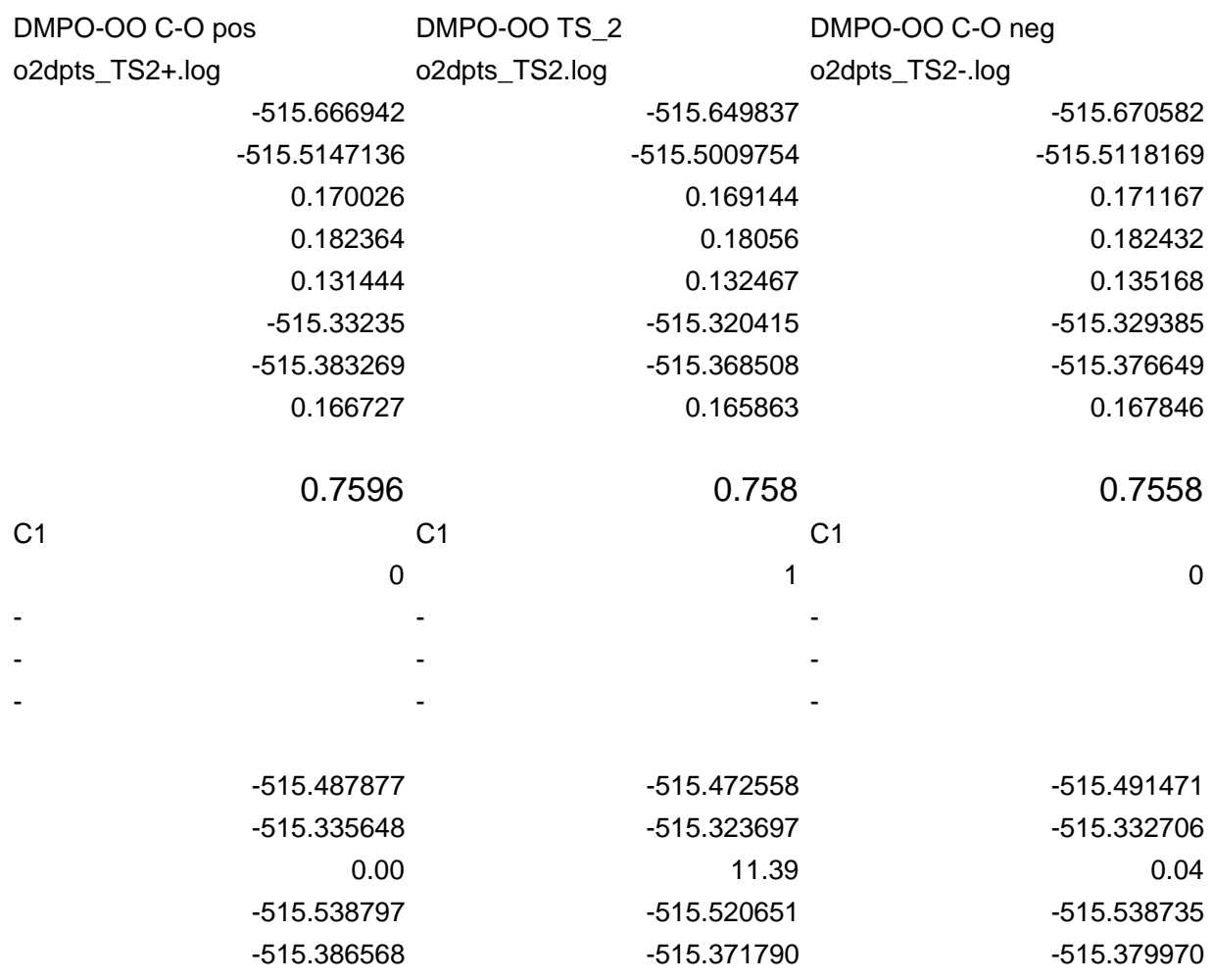



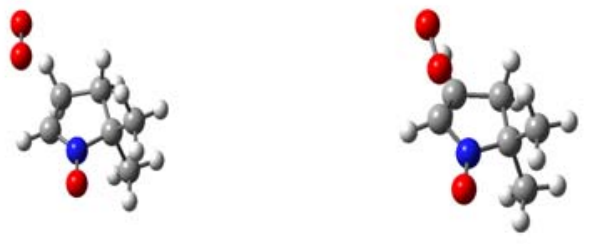

\begin{tabular}{lrr}
$\begin{array}{c}\text { DMPO-OO TS_3 } \\
\text { o2dpts_TS3.log }\end{array}$ & \multicolumn{2}{c}{ DMPO-OO C-O pos } \\
O2dpts_TS3+.log \\
\hline 5 & -515.650345 & -515.666942 \\
2 & -515.5038473 & -515.5155175 \\
7 & 0.169309 & 0.171201 \\
6 & 0.180651 & 0.182435 \\
6 & 0.132648 & 0.13532 \\
7 & -515.323197 & -515.333082 \\
4 & -515.3712 & -515.380198 \\
& 0.166024 & 0.167880 \\
& & \\
C1 & 0.7657 & 0.7574 \\
0 & \multicolumn{2}{c}{ C1 }
\end{tabular}

N Imag

Rotational Con. (GHz)

Rotational Con. (GHz)

Rotational Con. (GHz)

H298

E 6-31+g*t tight

G298

E 6-31+g*t tight

E 6-319*
$-515.486418$

$-515.339072$

0.00

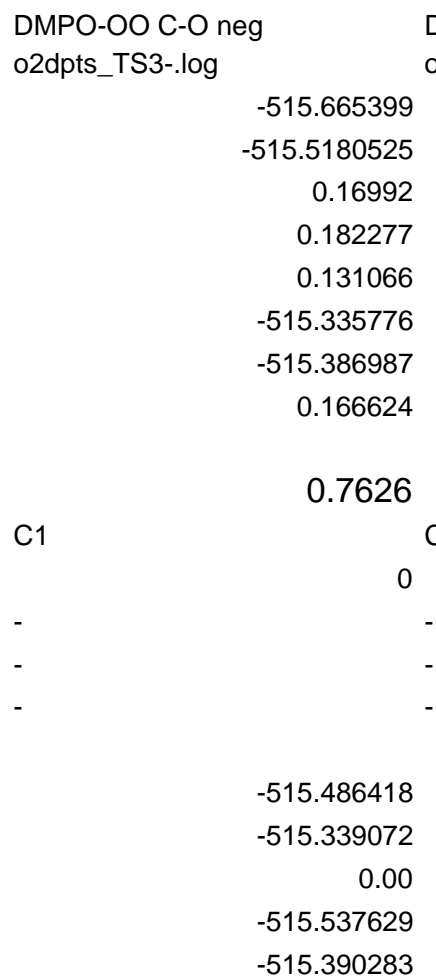

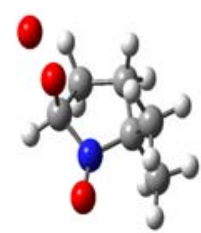

-

$\begin{array}{rr}-515.472979 & -515.487828 \\ -515.326481 & -515.336404 \\ 10.45 & 1.69 \\ -515.520982 & -515.534943 \\ -515.374484 & -515.383519\end{array}$




\section{䋈}

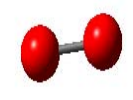

O2 Radical

02.log

$-365.208275$

$-365.1773173$

0.166744

0.175674

0.134895

$-365.001643$

$-365.042422$

0.163509

0.000000

s2 6-31g*

Symmetry

$N$ Imag

Rotational Con. (GHz)

Rotational Con. (GHz)

Rotational Con. (GHz)

H298

E 6-31+g** tight

E 6-31g*

G298

E 6-31+g** tight

E 6-31g*

\begin{tabular}{|c|c|}
\hline \multicolumn{2}{|c|}{$\begin{array}{l}\text { OOH Radical } \\
\text { peroxylrad.log }\end{array}$} \\
\hline-150.465075 & -150.926851 \\
\hline-150.2996938 & -150.899156 \\
\hline 0.002761 & 0.014031 \\
\hline 0.006082 & 0.017832 \\
\hline-0.017041 & -0.008160 \\
\hline-150.293612 & -150.881325 \\
\hline-150.316735 & -150.907317 \\
\hline 0.002707 & 0.013759 \\
\hline 0.7528 & 0.752700 \\
\hline \multicolumn{2}{|c|}{$\mathrm{C} 01$} \\
\hline 0 & 0.000000 \\
\hline \multicolumn{2}{|l|}{1.63425} \\
\hline \multicolumn{2}{|l|}{0.75785} \\
\hline \multicolumn{2}{|l|}{0.66048} \\
\hline-150.459047 & -150.909291 \\
\hline-150.293665 & -150.881597 \\
\hline-150.482170 & -150.935283 \\
\hline-150.316788 & -150.907589 \\
\hline
\end{tabular}




\begin{tabular}{lrr} 
Compound & OH \\
file & OHfreq.LOG & \\
E 6-31+g** tight (PCM) & & -75.747324 \\
E 6-31g* & -75.72215961 \\
ZPE (raw) & 0.008304 \\
Therm Corr to H (raw) & 0.011609 \\
Therm Corr to G (raw) & -0.008638 \\
H (raw) & -75.711846 \\
G (raw) & -75.732093 \\
ZPE (scaled) & 0.008143 \\
Electronic State & \\
s2 6-31g* & 0.751967 \\
Symmetry & \\
N Imag & & 0.000000 \\
Rotational Con. (GHz) & \\
Rotational Con. (GHz) & \\
Rotational Con. (GHz) & \\
H298 & & \\
E 6-31+g** tight & \\
E 6-319* & \\
G298 & \\
E 6-31+g** tight & -75.735876 \\
E 6-319* & -75.710712 \\
\hline
\end{tabular}




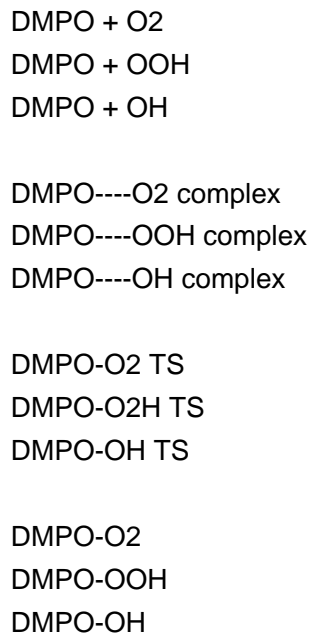

$\mathrm{DH}$

\begin{tabular}{ll}
\multicolumn{1}{c}{ DG } \\
-515.67335 & -515.558784 \\
-516.135126 & -516.011898 \\
-440.955599 & -440.832738 \\
& \\
-515.66653 & -515.5374503 \\
-516.143242 & -516.0023497 \\
-440.95854 & -440.8198036 \\
-515.648461 & -515.5177826 \\
-516.128933 & -515.9855446 \\
-440.959491 & -440.8200834 \\
-515.670531 & -515.5378725 \\
-516.165541 & -516.0192434 \\
-441.035643 & -440.8918707
\end{tabular}

$\mathrm{DH}$

DG 0.0 0.0 0.0
0.0
0.0
0.0
13.4
6.0
8.1
25.7
16.5
7.9
13.1
$-4.6$
$-37.1$ 
Table S9

Thermodynamic Parameters for the O-H Bond Dissociation of TEMPO-OH at the B3LYP/6-31+G(d,p)//B3LYP/6-31G(d), CBS-Q and CBS-QB3 levels in gas phase

and

CBS-QB3 Energies of the 3 Major DMPO-OOH Conformations 


\begin{tabular}{|c|c|c|c|}
\hline Molecule & $\mathrm{H}$ atom & 2,2,6,6-ТЕMPO & 2,2,6,6-ТЕМРОН \\
\hline E B3LYP/6-31G* & & -483.7197701 & -484.3260143 \\
\hline E B3LYP/6-31+G** & -0.500272784 & -483.7592508 & -484.3719768 \\
\hline ZPVE & 0 & 0.263322 & 0.275169 \\
\hline Thermal Corr. $\mathrm{H}$ & 0.00236 & 0.276304 & 0.288279 \\
\hline Thermal Corr. G & -0.010654 & 0.226574 & 0.239469 \\
\hline State & 2-A1G & $2-A^{\prime}$ & $1-\mathrm{A}^{\prime}$ \\
\hline$<$ S2 $>$ & 0.75 & 0.75 & 0 \\
\hline Point Group & $\mathrm{OH}$ & CS & CS \\
\hline Nimag. & 0 & 0 & 0 \\
\hline Rotational Constant A & & 1.47248 & 1.43551 \\
\hline Rotational Constant B & & 1.05699 & 1.07165 \\
\hline Rotational Constant C & & 0.84921 & 0.84731 \\
\hline Scaled ZPVE & 0 & 0.258213553 & 0.269830721 \\
\hline$\Delta \mathrm{E}(\mathrm{SCF})$ & & 70.6 & \\
\hline H (0 K) & & 63.3 & \\
\hline$\Delta H(298 \mathrm{~K})$ & & 64.7 & \\
\hline$\Delta G(298 \mathrm{~K})$ & & 55.9 & \\
\hline Molecule & $\mathrm{H}$ atom & 2,2,6,6-ТЕMPO & 2,2,6,6-ТЕМРОН \\
\hline CBS-Q (0 K) & -0.499818 & -482.806039 & -483.413818 \\
\hline CBS-Q Energy & -0.498402 & -482.794003 & -483.40161 \\
\hline CBS-Q Enthalpy & -0.497457 & -482.793059 & -483.400665 \\
\hline CBS-Q Free Energy & -0.510472 & -482.842723 & -483.449568 \\
\hline \multicolumn{4}{|l|}{ Thermal Corr. G } \\
\hline State & & $2-A^{\prime}$ & $1-\mathrm{A}^{\prime}$ \\
\hline$<$ S2 $>$ & & 0.77 & 0 \\
\hline Point Group & & CS & CS \\
\hline Nimag. & & 0 & 0 \\
\hline Rotational Constant A & & 1.4822906 & 1.4481716 \\
\hline Rotational Constant B & & 1.0718704 & 1.0869863 \\
\hline Rotational Constant C & & 0.8600131 & 0.8590002 \\
\hline H (0 K) & & 67.7 & \\
\hline$\Delta H(298 \mathrm{~K})$ & & 69.1 & \\
\hline$\Delta G(298 \mathrm{~K})$ & & 60.5 & \\
\hline Molecule & H atom & 2,2,6,6-ТЕМРО & 2,2,6,6-ТЕМРОН \\
\hline CBS-QB3 (0 K) & -0.499818 & -482.795283 & -483.404664 \\
\hline CBS-QB3 Energy & -0.498402 & -482.783154 & -483.392396 \\
\hline CBS-QB3 Enthalpy & -0.497457 & -482.782209 & -483.391451 \\
\hline CBS-QB3 Free Energy & -0.510472 & -482.832036 & -483.440392 \\
\hline \multicolumn{4}{|l|}{ Thermal Corr. G } \\
\hline State & & $2-A^{\prime}$ & $1-\mathrm{A}^{\prime}$ \\
\hline$<$ S2 $>$ & & 0.77 & 0 \\
\hline Point Group & & CS & CS \\
\hline Nimag. & & 0 & 0 \\
\hline Rotational Constant A & & 1.475943 & 1.4383993 \\
\hline Rotational Constant B & & 1.0571469 & 1.0724501 \\
\hline Rotational Constant C & & 0.8498351 & 0.8479871 \\
\hline H (0 K) & & 68.8 & \\
\hline$\Delta H(298 \mathrm{~K})$ & & 70.1 & \\
\hline$\Delta G(298 \mathrm{~K})$ & 5100 & 61.4 & \\
\hline
\end{tabular}


Molecule
CBS-QB3 (0 K)
CBS-QB3 Energy
CBS-QB3 Enthalpy
CBS-QB3 Free Energy

DMPO-OOH 180

$-515.309712$

$-515.298681$

$-515.297737$

$-515.346461$
DMPO-OOH 294

$-515.309314$

$-515.29816$

$-515.297216$

$-515.346394$
DMPO-OOH 76

$-515.31397$

$-515.303339$

$-515.302395$

$-515.350206$ 